$$
\begin{aligned}
& \text { SH } \\
& 361 \\
& .44 \\
& 1922
\end{aligned}
$$

u. 8. Bureau of fist

Fur seal skin

sales 


6

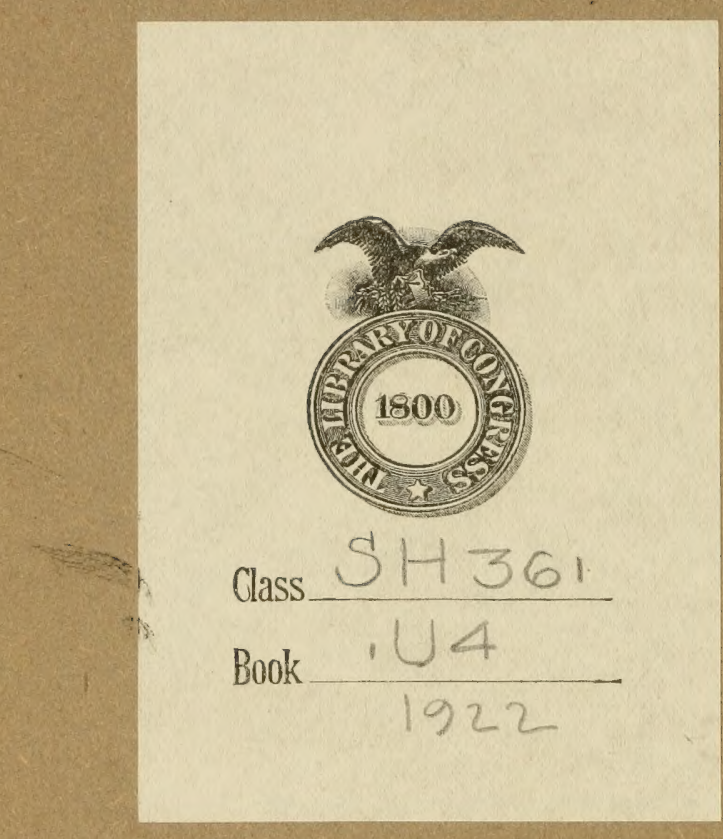




\section{FUR-SEAL SKIN SALES}

\section{LETTER FROM \\ THE SECRETARY OF COMMERCE}

TRANSMITTING

IN RESPONSE TO A SENATE RESOLUTION OF APRIL 20 (CALEN-

DAR DAY, MAY 27), 1922, A STATEMENT RELATIVE TO GOVERNMENT-OWNED ALASKA FUR-SEAL SKINS; TOTAL

ANNUAL PAYMENTS TO THE GOVERNMENTS OF

GREAT BRITAIN AND JAPAN SINCE 1912, UNDER

ACT AUGUST 24, 1912, AND COPIES OF GOV-

ERNMENT CONTRACTS FOR DRESSING

AND DYEING FUR-SEAL SKINS

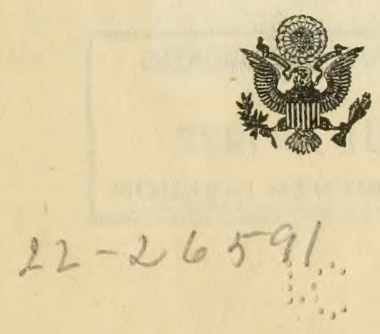

April 20 (calendar day, June 17), 1922.-Ordered to lie on the table

WASHINGTON

GOVERNMENT PRINTING OFFICE 
In the Senate of the United States, A pril 20 (calendar day, June 20), 1922.

Ordered, That the communication of the Secretary of Commerce, relative to the total number and prices of Government-owned Alaska fur-seal skins taken and sold, transmitted to the Senate on Saturday, the 17 th instant, in response to Senate Resolution 287, be printed together with the accompanying papers, as a Senate document.

Attest:

George A. SANDERson,

Secretary.

u

LIBRARY OF CONGRESS

RECEIVED

3L1L15192\%

BQOUMENTS DIVISION

$\because \vdots$ 


\section{LETTER OF TRANSMITTAL.}

\section{Department of Commerce, Office of the Secretary, Washington, June 17, 1922.}

The President of the United States Senate,

$$
\text { Washington, D. C. }
$$

SIR: I have the honor to transmit herewith a statement from the Commissioner of Fisheries in response to a resolution of the Senate of the United States, passed May 27, 1922, directing the Secretary of Commerce to furnish the Senate a statement showing the total number of Government-owned Alaska fur-seal skins taken, and other information in connection therewith, together with a complete record of the total annual payments made to the Governments of Great Britain and Japan since 1912, to January 1, 1922, inclusive, and copies of Government contracts made with certain fur companies for dressing and dyeing fur-seal skins owned by the Government. Yours faithfully,

Herbert Hoover. 



\title{
FUR-SEAL SKIN SALES.
}

\author{
Department of Commerce, \\ Bureau of Fisheries, \\ Washington, June 16, 1922.
}

Subject: Senate resolution passed May 27, 1922, in regard to Government-owned Alaska sealskins.

The Secretary of Commerce: In compliance with your instructions relative to Senate resolution directing the Secretary of Commerce to furnish the Senate a statement showing in detail the number of Government-owned Alaska fur-seal skins taken and sold, and other information connected therewith, there are transmitted herewith Statements I to XIX, showing the number of fur-seal skins taken annually on the Pribilof Islands, and the disposition thereof, together with detailed information showing time, place, number of skins sold, classification of skins sold, and prices obtained for each grade and classification.

There are also inclosed Statements XX to XXVIII, showing the amounts paid the Governments of Great Britain and Japan since 1912 to January 1, 1922, together with photostat copies of all contracts and supplemental agreements with Funsten Bros. \& Co. and the Fouke Fur. Co., for dressing and dyeing fur-seal skins owned by the Government. In this connection attention is repectfully called to the following statement of comparison of revenue received by the United States Government from the Pribilof Islands, Alaska, under leases from 1870 to 1909 , and under direct management by the Department of Commerce from 1910 to 1921 :

Comparison of revenue received by the United States Government from Pribilof Islands, Alaska, under leases and under direct management by the Department of Commerce.

Under exclusive sealing rights by lease to Alaska Commercial Co., 1870 to 1899 :

Revenue from 1,840,364 skins and lease.

$\$ 6,010,565.00$

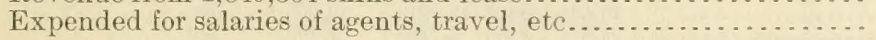
$202,655.00$

Net revenue received.

$5,807,910.00$

Net average per skin, $\$ 3.15$.

Under exclusive rights by lease to North American Commercial Co., 1890 to 1909 :

Revenue from 339,180 skins and lease.................... $\$ 3,752,415.00$

Expended for salaries, travel, food for natives, etc.............. $596,085.00$

Net revenue received.......................... 3, 156, 330.00 Net average per skin, $\$ 9.30$.

Under direct management by the Department of Commerce, 1910 to July 1, 1921:

Revenue from 101,594 skins...................... $\$ 4,321,141.03$

Expended for salaries, travel, care of natives, etc ............... 1, 151,596.50

Net revenue received............................. ${ }^{1} 3,169,544.53$ Net average per skin, $\$ 31.20$.

${ }^{1}$ No deductions have been made from this total for payments to Great Britain and Japan, amounting to $\$ 505,434.64$ each. 
Under the leasing arrangements the Government received no revenue from the sale of fox skins from the Pribilof Islands. Under the direct management of the Department of Commerce since 1910 there have been sold 5,204 fox skins, which netted the Government $\$ 403,759.34$.

Attention is also called to the fact that when the Alaska Commercial Co. leased the right to take sealskins on the Pribilof Islands, the seal herd was estimated to contain over 2,000,000 animals, and during the leasing period of 40 years the herd was so depleted as to leave only 132,279 animals when the direct management of the islands was taken over, in 1910, by the Department of Commerce. From that date, to and including 1921, the stock has been increased to 581,453 animals, and there are now 41,091 sealskins on hand.

The average net price per skin received by the Government during the 40 years under lease is $\$ 4.11$. The net increase in price per skin received under direct management by the Department of Commerce is over 600 per cent, and during this period the stock of animals and skins on hand, instead of being reduced, as under the leasing periods, has been built up and increased over 370 per cent.

$$
\begin{gathered}
\text { Henry O'Malley, } \\
\text { Commissioner. }
\end{gathered}
$$

\section{I.}

Tabular statement showing receipts and disposition of fur-seal skins taken on the Pribilof Islands by the Department of Commerce, 1912 to 1921.

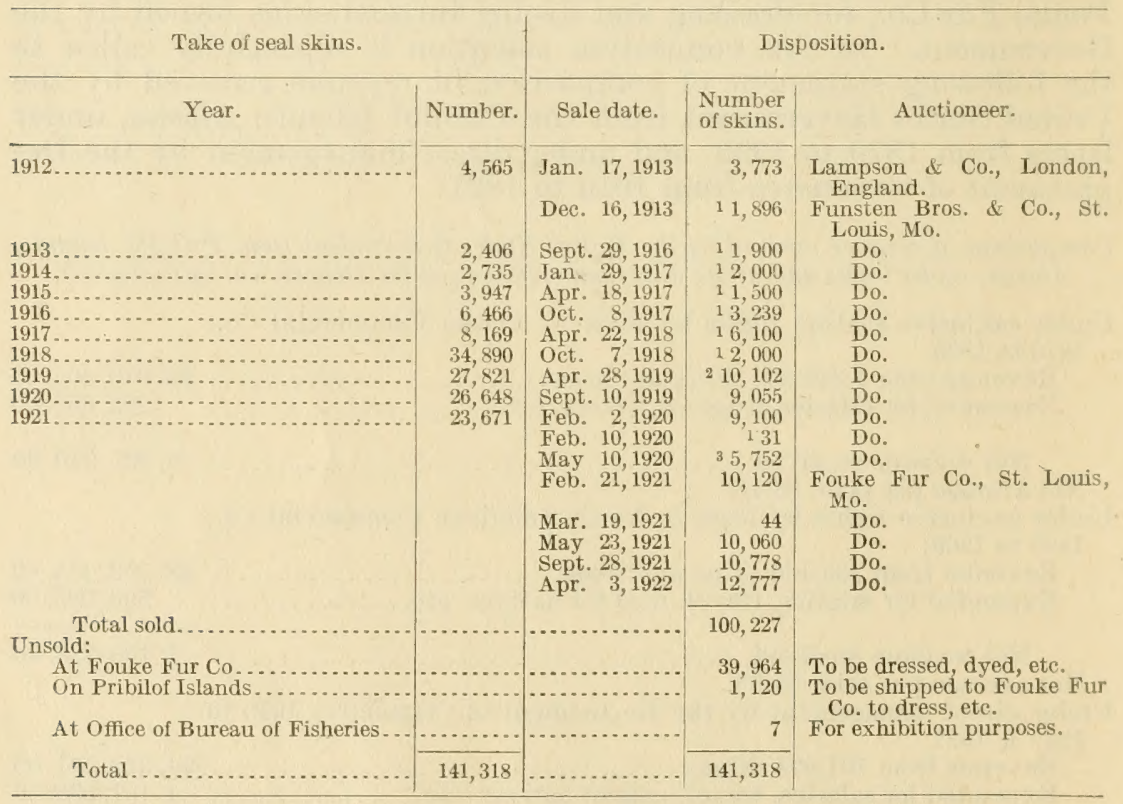

1 By the terms of the sealing convention of July 17, 1911, and the act of August 24, 1912, Great Britain and Japan, during the period suspending the killing of fur seals for commercial purposes, each received a loan of $\$ 10,000$ per annum in lieu of any financial interest in the seals killed for food purposes. The skins in this sale were from seals taken for food of the natives of the Pribilof Islands and not for commercial purposes.

2 Of these 10,102 skins, 3,882 were food skins of 1917 take; 1,943 were commercial skins of 1917 take, and 4,277 were commercial skins of 1918 take.

3 Twelve of these were food skins taken in 1917 with which the foreign countries had no financial interest 
II.

Details of sale of 3,773 salted Pribilof Islands fur-seal skins at London, England, on January 17, 1913.

\begin{tabular}{|c|c|c|c|c|}
\hline Trade classification. & $\begin{array}{l}\text { Lot } \\
\text { No. }\end{array}$ & $\begin{array}{l}\text { Number } \\
\text { of } \\
\text { skins. }\end{array}$ & $\begin{array}{l}\text { Price } \\
\text { per } \\
\text { skin. }\end{array}$ & $\begin{array}{l}\text { Total } \\
\text { for lot. }\end{array}$ \\
\hline 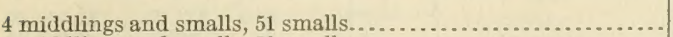 & 1 & 55 & 856.60 & $\$ 3.113,00$ \\
\hline 4 middlings and smalls, 50 smalls. . . . . . . . . . . . . . . . . . . . . . . & 2 & 54 & 56.60 & $3,056.40$ \\
\hline 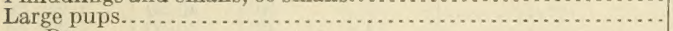 & 3 & 80 & 51.23 & $4,098.40$ \\
\hline (1) & 4 & 80 & 51.72 & $4,137.60$ \\
\hline . & 5 & 80 & 51. 72 & $4,137.60$ \\
\hline 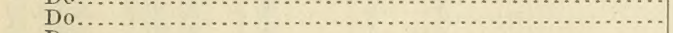 & 6 & 80 & 51. 72 & $4,137.60$ \\
\hline (n) & 7 & 80 & 51.72 & $4,137.60$ \\
\hline (2) & 8 & 66 & 51.71 & $3,412.86$ \\
\hline (n) & 9 & 90 & 41.97 & $3,777.30$ \\
\hline 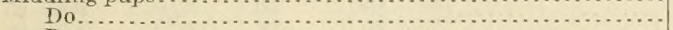 & 10 & 90 & 41.97 & $3,777.30$ \\
\hline 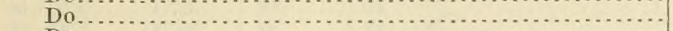 & 11 & 90 & 42.94 & $3,864.60$ \\
\hline 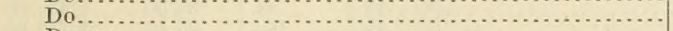 & 12 & 90 & 42.94 & $3,864.60$ \\
\hline 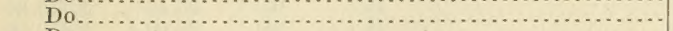 & 13 & 90 & 43.92 & $3,952.80$ \\
\hline . & 14 & 90 & 42.94 & 64.60 \\
\hline (n. & 15 & 90 & 43. 42 & 97.80 \\
\hline 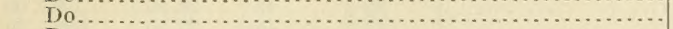 & 16 & 90 & 43.42 & 7. 80 \\
\hline 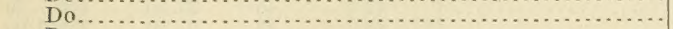 & 17 & 90 & 43.42 & 07.80 \\
\hline 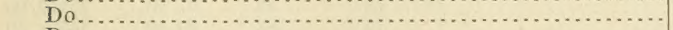 & 18 & 90 & 43.42 & 97.80 \\
\hline 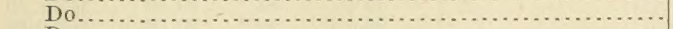 & 19 & 90 & 43. 42 & 08.70 \\
\hline 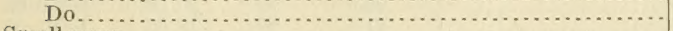 & 20 & 57 & 43. 44 & 76.08 \\
\hline$\ldots \ldots \ldots \ldots \ldots \ldots \ldots \ldots \ldots \ldots$ & 21 & 100 & 31.72 & $3,172.00$ \\
\hline 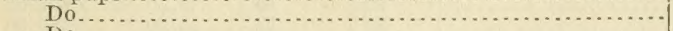 & 22 & 100 & 31.72 & $3,172.00$ \\
\hline 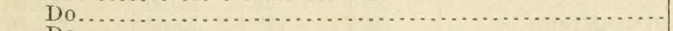 & 23 & 100 & 31.72 & $3,172.00$ \\
\hline 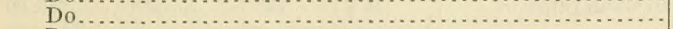 & 24 & 100 & 31.72 & $3,172.00$ \\
\hline (n. & 25 & 100 & 31.72 & $31,72.00$ \\
\hline${ }^{\prime} \ldots \ldots \ldots \ldots \ldots \ldots \ldots \ldots \ldots \ldots \ldots \ldots \ldots$, & 26 & 100 & 32.20 & $3,220.00$ \\
\hline 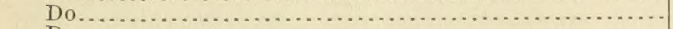 & 27 & 100 & 31.72 & $3,172.00$ \\
\hline 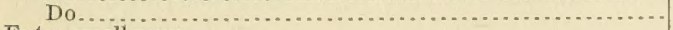 & 28 & 76 & 31.72 & $2,410.72$ \\
\hline Extra small pups. . & 29 & 37 & 25.85 & 956.45 \\
\hline 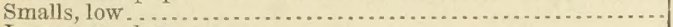 & 30 & 56 & 38.55 & $2,158.80$ \\
\hline 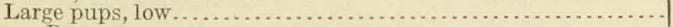 & 31 & 62 & 35.13 & $2,178.06$ \\
\hline$\ldots \ldots \ldots \ldots \ldots \ldots \ldots \ldots$ & 32 & 62 & 35.13 & $2,178.06$ \\
\hline ........ & 33 & 62 & 35. 13 & $2,178.06$ \\
\hline$\ldots \ldots \ldots \ldots \ldots \ldots \ldots \ldots \ldots$ & 34 & 81 & 27.33 & $2,213.73$ \\
\hline 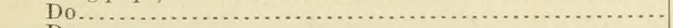 & 35 & 81 & 26.84 & $2,174.04$ \\
\hline$\ldots \ldots \ldots \ldots$. & 36 & 81 & 26.84 & $2,174.04$ \\
\hline 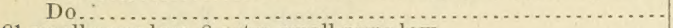 & 37 & 80 & 26.84 & $2,147.20$ \\
\hline 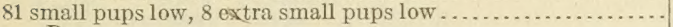 & 38 & 89 & 20.50 & $1,824.50$ \\
\hline Do & 39 & 89 & 20.50 & $1,824.50$ \\
\hline 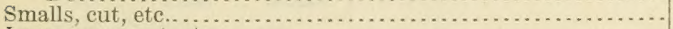 & 40 & 37 & 50.75 & $1,877.75$ \\
\hline Large pups, cut, ete. . . . . . . . . . . . . . & 41 & 70 & 44. 40 & $3,108: 00$ \\
\hline & 42 & 69 & 44. 40 & $3,063.60$ \\
\hline Middling pups, cut, etc.. & $=43$ & 70 & 31.23 & $2,186.10$ \\
\hline ................... & 44 & 70 & 31.23 & $2,186.10$ \\
\hline D & 45 & 71 & 31.23 & $2,217.33$ \\
\hline 93 small p & 46 & 98 & 23.42 & $2,295.16$ \\
\hline 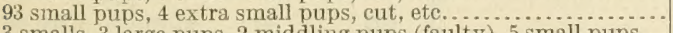 & 47 & 97 & 23.42 & $2,271.74$ \\
\hline \multirow{2}{*}{ 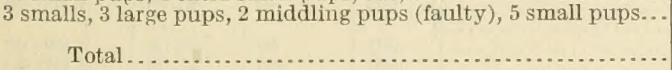 } & 48 & 13 & 5.10 & \\
\hline & & 3,773 & & $141,289.58$ \\
\hline
\end{tabular}


III.

Details of sale of 1,898 salted Pribilof Islands fur-seal skins at St. Louis, Mo., December $16,1913$.

\begin{tabular}{|c|c|c|c|c|}
\hline Trade classification. & $\begin{array}{l}\text { Lot } \\
\text { No. }\end{array}$ & $\begin{array}{l}\text { Number } \\
\text { of } \\
\text { skins. }\end{array}$ & $\begin{array}{l}\text { Price } \\
\text { per } \\
\text { skin. }\end{array}$ & $\begin{array}{l}\text { Total } \\
\text { for lot. }\end{array}$ \\
\hline 2 middlings and small; 39 small . .................. & 1 & 41 & $\$ 52.00$ & $\$ 2,132.00$ \\
\hline 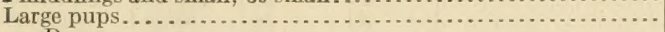 & 2 & 80 & 40.50 & $3,240.00$ \\
\hline 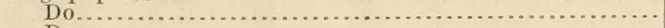 & $\overline{3}$ & 80 & 40.50 & $3,240.00$ \\
\hline 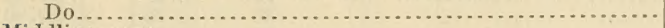 & 4 & 43 & 40.00 & $1,720.00$ \\
\hline 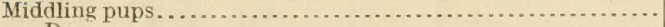 & $\overline{5}$ & 90 & 31.50 & $2,835.00$ \\
\hline 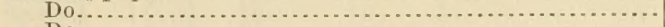 & 6 & 90 & 33.50 & 3.015 .00 \\
\hline 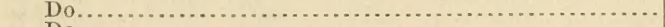 & 7 & 90 & 34.00 & $3,060.00$ \\
\hline 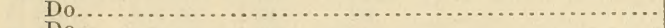 & 8 & 90 & 34,00 & $3,060.00$ \\
\hline 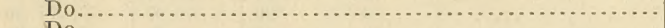 & 9 & 90 & 32,50 & $2,925,00$ \\
\hline Do & 10 & 90 & 32.50 & 2, 925.00 \\
\hline Do & 11 & 90 & 32.50 & $2,925.00$ \\
\hline Small pups.... & 12 & 44 & 34.00 & $1,496,00$ \\
\hline 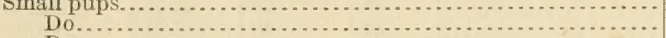 & 13 & 100 & $\begin{array}{l}25.50 \\
25.50\end{array}$ & $2,550.00$ \\
\hline Do & 15 & 82 & 25.50 & $2,091.00$ \\
\hline 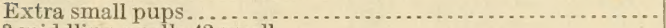 & 16 & 18 & 26.50 & 477.00 \\
\hline 3 middling small; 42 small . . . . . . . . . . . . . . . . . . . . . . . . . . . & 17 & 45 & 23.50 & $1,057.50$ \\
\hline Large pups. . . . . . . . . . . . . . . . . . . . . . . . . . . . . . . . . & 18 & 88 & 21.50 & $1,892.00$ \\
\hline 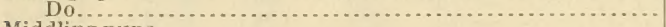 & 19 & 83 & 20.50 & $1,701.50$ \\
\hline 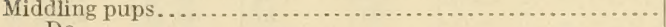 & 20 & 89 & 20.00 & $1,780.00$ \\
\hline 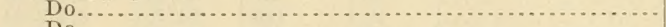 & 21 & 89 & 19.00 & $1,691.00$ \\
\hline 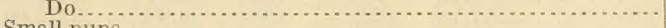 & 22 & 80 & 19.50 & $1,560.00$ \\
\hline 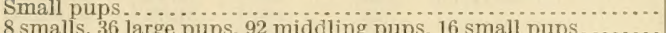 & 23 & 52 & 15.00 & 780.00 \\
\hline \multirow{2}{*}{ 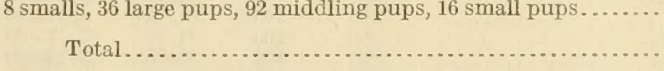 } & 24 & 152 & 25.50 & $3,876.00$ \\
\hline & & 1,898 & & $54,579.00$ \\
\hline
\end{tabular}

IV.

Details of sale of 1,900 dressed, dyed, and machined Pribilof Islands fur-seal skins at St. Louis, September 20, 1916.

\begin{tabular}{|c|c|c|c|c|}
\hline Trade classification. & $\begin{array}{l}\text { Lot } \\
\text { No. }\end{array}$ & $\begin{array}{c}\text { Number } \\
\text { of } \\
\text { skins. }\end{array}$ & $\begin{array}{l}\text { Price } \\
\text { per } \\
\text { skin. }\end{array}$ & $\begin{array}{l}\text { Total } \\
\text { for lot. }\end{array}$ \\
\hline 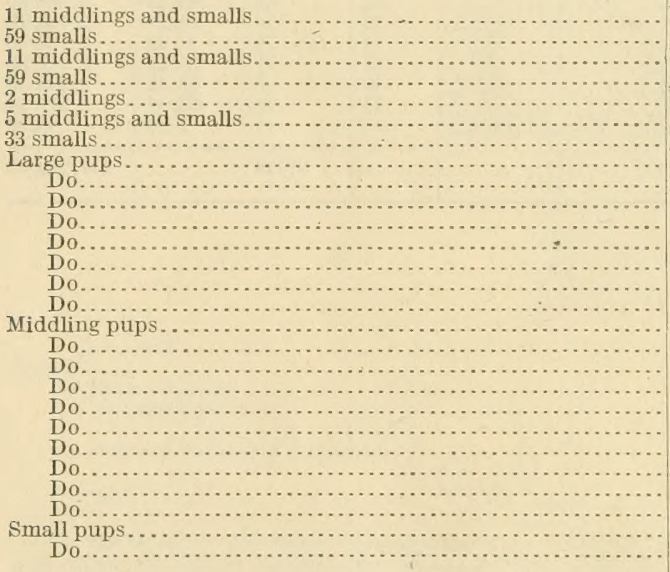 & $\begin{array}{l}502 \\
503 \\
504 \\
505 \\
506 \\
507 \\
508 \\
509 \\
510 \\
511 \\
512 \\
513 \\
514 \\
515 \\
516 \\
517 \\
518 \\
519 \\
520 \\
521 \\
522\end{array}$ & $\begin{array}{l}40 \\
80 \\
80 \\
80 \\
80 \\
80 \\
80 \\
80 \\
80 \\
90 \\
90 \\
90 \\
90 \\
90 \\
90 \\
90 \\
90 \\
90 \\
90 \\
90 \\
90\end{array}$ & $\begin{array}{l}\$ 50.00 \\
51.00 \\
45.00 \\
36.00 \\
37.00 \\
39.00 \\
42.00 \\
39.00 \\
40.00 \\
43.00 \\
43.00 \\
36.00 \\
38.00 \\
38.00 \\
39.00 \\
39.00 \\
35.00 \\
36.00 \\
36.00 \\
38.00 \\
40.00 \\
35.00 \\
36.00\end{array}$ & $\begin{array}{c}\$ 3,500.00 \\
3,570.00 \\
1,800.00 \\
2,880.00 \\
2,960.00 \\
3,120.00 \\
3,360.00 \\
3,120.00 \\
3,200.00 \\
3,440.00 \\
3,440.00 \\
3,240.00 \\
3,420.00 \\
3,420.00 \\
3,510.00 \\
3,510.00 \\
3,150.00 \\
3,240.00 \\
3,240.00 \\
3,420.00 \\
3,600.00 \\
3,150.00 \\
3,240.00\end{array}$ \\
\hline $\begin{array}{l}n^{2} \\
n_{1}\end{array}$ & & 1,900 & & $74,530.00$ \\
\hline
\end{tabular}


V.

Details of sale of 2,000 dressed, dyed, and machined Pribilof Islands fur-seal skins at St. Louis, Mo., January 29, 1917.

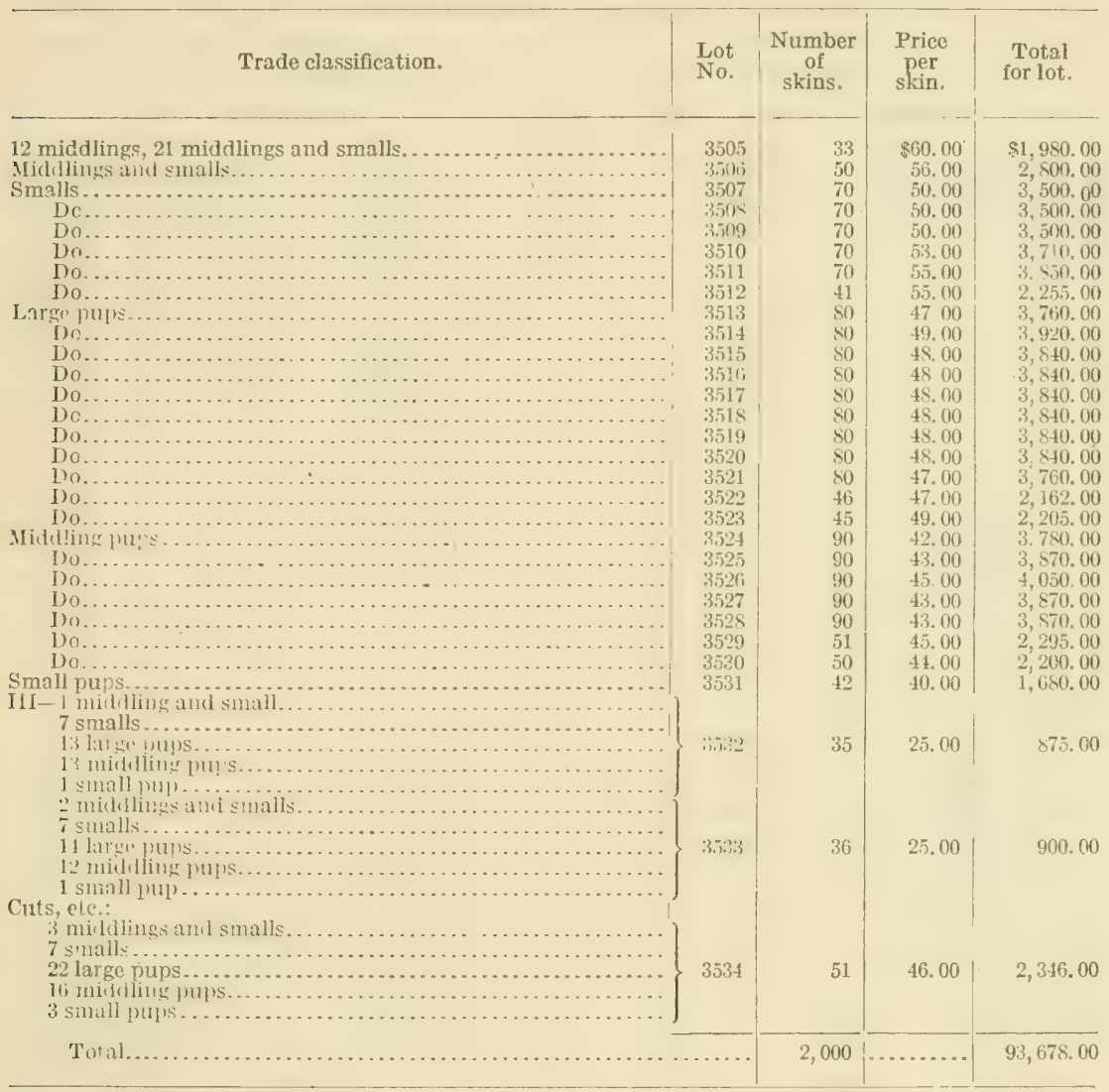


VI.

Details of sale of 1,500 dressed, dyed and machined Pribilof Islands fur-seal skins at St. Louis, April 18, $191 \%$.

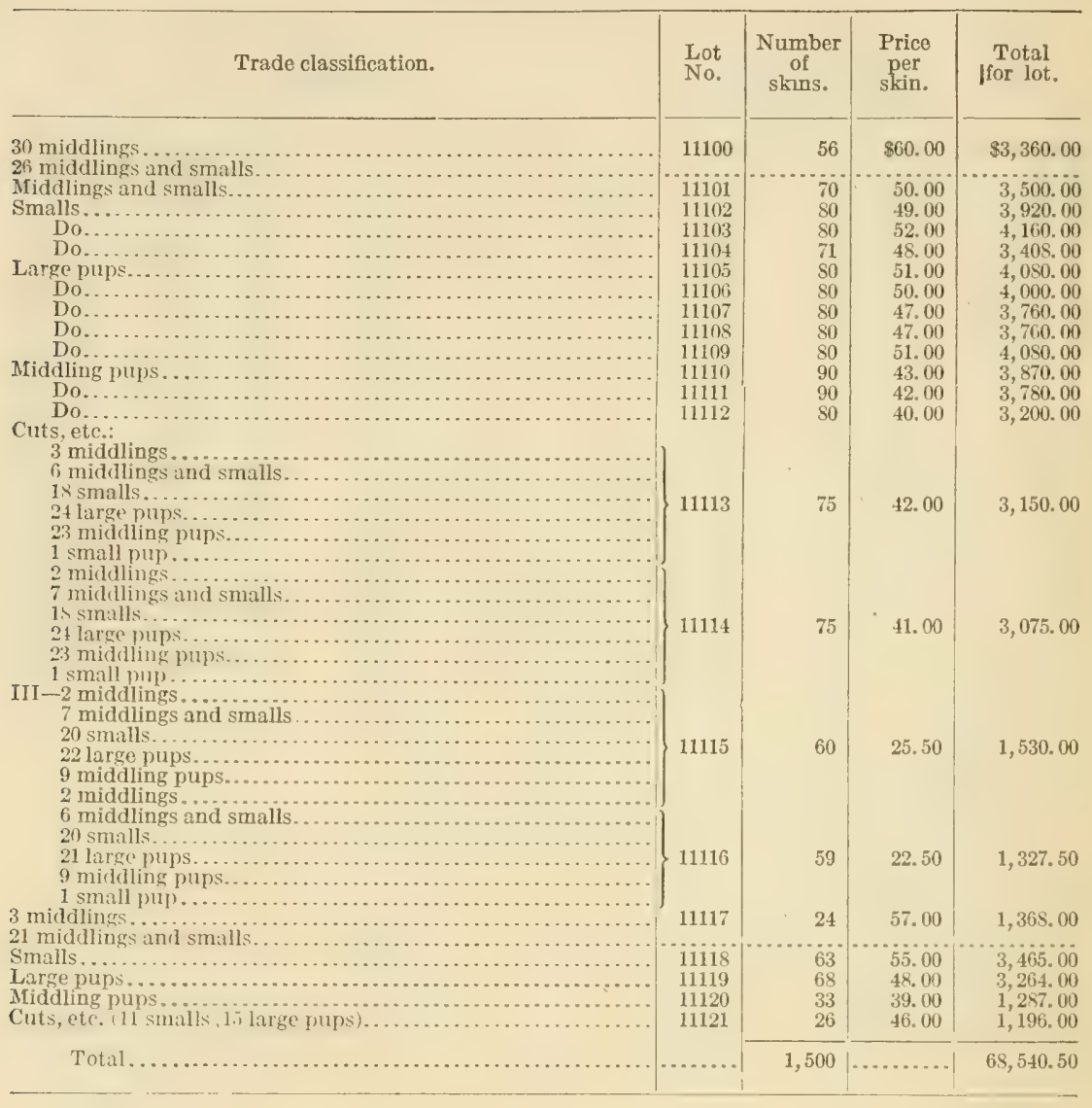


VII.

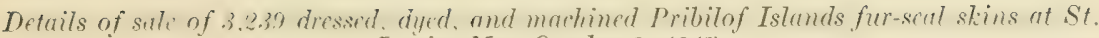
Louis, Mo., October 8, 1917.

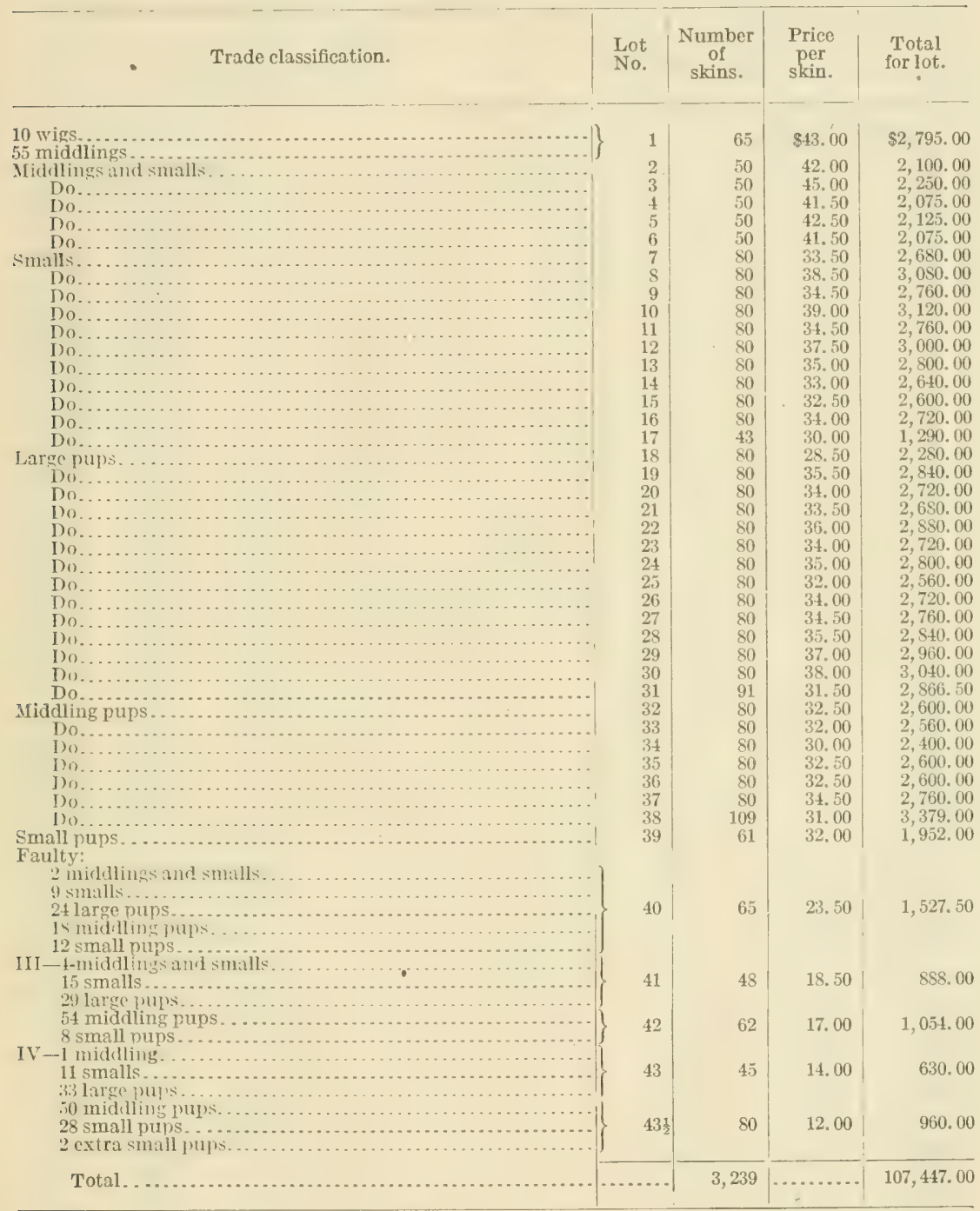


VIII.

Details of sale of 6,100 dressed, dyed, and machined Pribilof Islands fur-seal skins at St: Louis, Mo., April 22, 1918.

\begin{tabular}{|c|c|c|c|c|}
\hline Trade classification. & $\begin{array}{l}\text { Lot } \\
\text { No. }\end{array}$ & $\begin{array}{c}\text { Number } \\
\text { of } \\
\text { skins. }\end{array}$ & $\begin{array}{l}\text { Price } \\
\text { per } \\
\text { skin. }\end{array}$ & $\begin{array}{l}\text { Total } \\
\text { for lot. }\end{array}$ \\
\hline 15 wigs; 50 extra extra large. & 1 & 65 & $\$ 64.00$ & $\$ 4,160,00$ \\
\hline 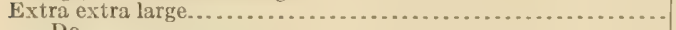 & 2 & 50 & 57.00 & $2,850.00$ \\
\hline 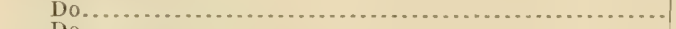 & 3 & 50 & 58.00 & $2,900.00$ \\
\hline 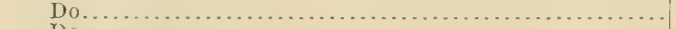 & 4 & 50 & 56.00 & $2,800.00$ \\
\hline 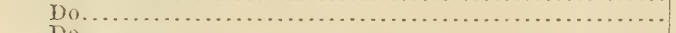 & 5 & 50 & 57. 00 & $2,850,00$ \\
\hline 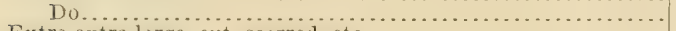 & 6 & 50 & 56. 00 & $2,800,00$ \\
\hline 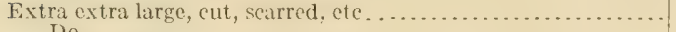 & 7 & 50 & 40. 00 & $2,000.00$ \\
\hline 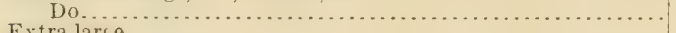 & 8 & 40 & 43. 00 & $1,720.00$ \\
\hline 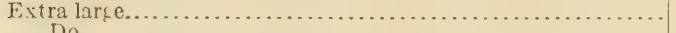 & 9 & 70 & 51,00 & $3,570.00$ \\
\hline 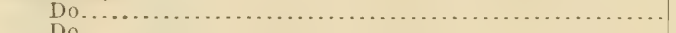 & 10 & 70 & 51.00 & $3,570.00$ \\
\hline 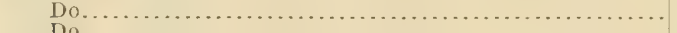 & 11 & 70 & 54,00 & $3,780.00$ \\
\hline 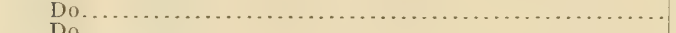 & 12 & 70 & 56.00 & $3,920,00$ \\
\hline 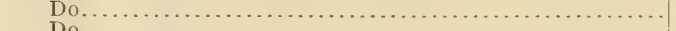 & 13 & 70 & 56.00 & $3,920,00$ \\
\hline 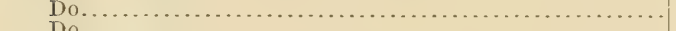 & 14 & 70 & 53.00 & $3,710.00$ \\
\hline$\ldots \ldots \ldots \ldots \ldots \ldots \ldots \ldots \ldots \ldots \ldots \ldots \ldots$ & 15 & 70 & 57.00 & $3,990,00$ \\
\hline$\ldots \ldots \ldots \ldots \ldots \ldots \ldots \ldots \ldots \ldots \ldots \ldots \ldots$ & 16 & 70 & 57. 00 & $3,990.00$ \\
\hline$\ldots \ldots \ldots \ldots \ldots \ldots \ldots \ldots \ldots \ldots \ldots \ldots \ldots$ & 17 & 70 & 54.00 & $3,780.00$ \\
\hline$\ldots \ldots \ldots \ldots \ldots \ldots \ldots \ldots \ldots \ldots \ldots \ldots$, & 18 & 70 & 56.00 & $3,920.00$ \\
\hline 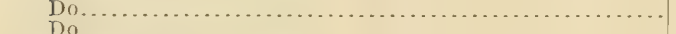 & 19 & 70 & 53.00 & .00 \\
\hline$\ldots \ldots \ldots \ldots \ldots \ldots \ldots \ldots$ & 20 & 60 & 54.00 & $3,240,00$ \\
\hline$\ldots \ldots \ldots \ldots \ldots \ldots \ldots \ldots \ldots \ldots \ldots$ & 21 & 50 & 35.00 & $1,750.00$ \\
\hline 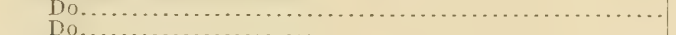 & 22 & 50 & 00 & 0.00 \\
\hline 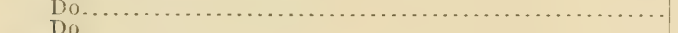 & 23 & 50 & 36.50 & 00 \\
\hline 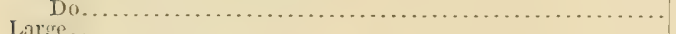 & 21 & 40 & 37.50 & 00 \\
\hline 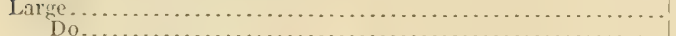 & 25 & 80 & 48.00 & 0.00 \\
\hline 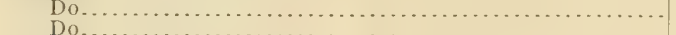 & 26 & 80 & 0 & .00 \\
\hline 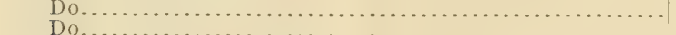 & 27 & 80 & 0 & 0.00 \\
\hline 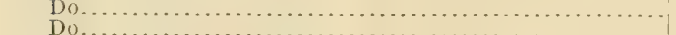 & $2 \mathrm{~S}$ & 80 & 51.00 & $4,050,00$ \\
\hline 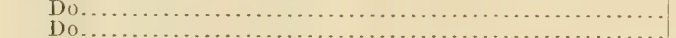 & 29 & 80 & 0 & .00 \\
\hline 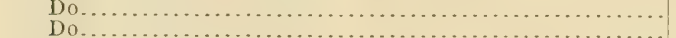 & 30 & 80 & 0 & .00 \\
\hline 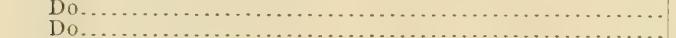 & 31 & 80 & 0 & .00 \\
\hline & 32 & 80 & 0 & 00 \\
\hline 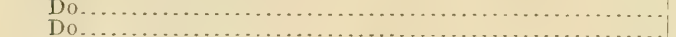 & 33 & 80 & 0 & 00 \\
\hline 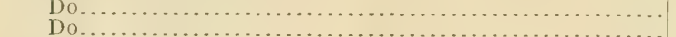 & 34 & 80 & 0 & 00 \\
\hline n. & 35 & 80 & 0 & $4,240.00$ \\
\hline$\cdots \cdots \cdots \cdots \cdots$ & 36 & 80 & 52. & $4,160.00$ \\
\hline (1, & 37 & 80 & 54. & $4,360,00$ \\
\hline${ }_{1}$ & 38 & 80 & 52 & 4 \\
\hline 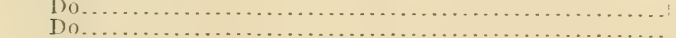 & 39 & 80 & 5.3 & 4,2 \\
\hline${ }^{\prime}{ }_{1}$ & 40 & 80 & 52 & 4,16 \\
\hline 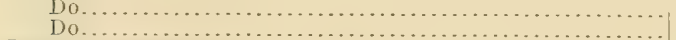 & 41 & 80 & 53 & $4,240.00$ \\
\hline 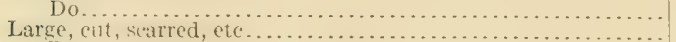 & 42 & 80 & 52.00 & $4,160.00$ \\
\hline (n. & 43 & 80 & 35.00 & $2,800,00$ \\
\hline 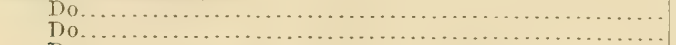 & 44 & 80 & 33.00 & $2,640,00$ \\
\hline 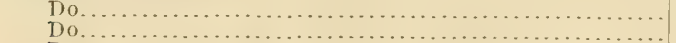 & 45 & 80 & 36. & $2,880.00$ \\
\hline 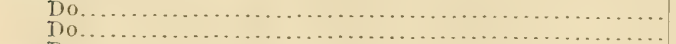 & 46 & 80 & 37. & $2,960.00$ \\
\hline 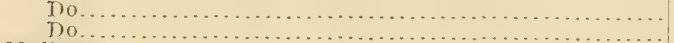 & 47 & 80 & 36.00 & $2,880.00$ \\
\hline 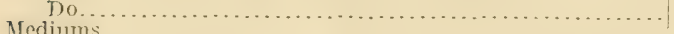 & 48 & 60 & 37.50 & $2,250,00$ \\
\hline 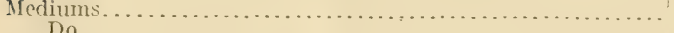 & 49 & 90 & 43.00 & $3,870.00$ \\
\hline 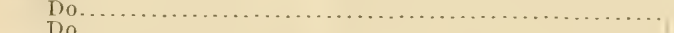 & 50 & 90 & 40.50 & $3,645.00$ \\
\hline$\ldots \ldots \ldots \ldots \ldots \ldots \ldots \ldots \ldots \ldots$ & 51 & 90 & 40.50 & $3,645.00$ \\
\hline$\ldots \ldots \ldots \ldots \ldots \ldots \ldots \ldots \ldots \ldots$ & 52 & 90 & 39.00 & $3,510.00$ \\
\hline$\ldots \ldots \ldots \ldots \ldots \ldots \ldots \ldots \ldots \ldots \ldots$ & 53 & 90 & 41.50 & $3,735.00$ \\
\hline$\ldots \ldots \ldots \ldots \ldots \ldots$ & 54 & 90 & 41. & $3,690.00$ \\
\hline Do.............. & & 90 & 39. & $3,510.00$ \\
\hline 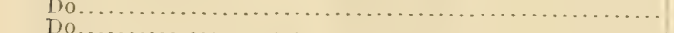 & 56 & 90 & 40. & $3,600.00$ \\
\hline 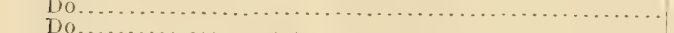 & 57 & 90 & 41. & $3,690.00$ \\
\hline 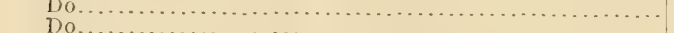 & 58 & 90 & 41 & $3,690.00$ \\
\hline 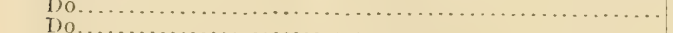 & 59 & 90 & 43 & $3,870.00$ \\
\hline 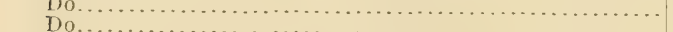 & 60 & 90 & 40. & 3,60 \\
\hline 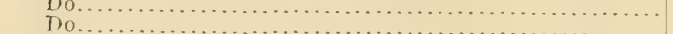 & 61 & 90 & 43 & $3,915.00$ \\
\hline & 62 & 90 & 45 & $4,095.00$ \\
\hline (n. & 6 & 90 & 45 & $4,050.00$ \\
\hline$\cdots \ldots \ldots \ldots \ldots \ldots \ldots \ldots \ldots$ & 64 & 90 & 43. & $3,870.00$ \\
\hline - . ona & 65 & 90 & 44. & $4,005,00$ \\
\hline searred, & 66 & 90 & 44. & $4,005.00$ \\
\hline ut, searred, & 67 & 90 & 33. & $2,970.00$ \\
\hline ( & 68 & 90 & 34. & $3,060.00$ \\
\hline 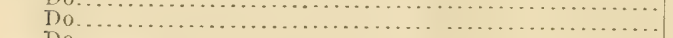 & 69 & 90 & 31. & $2,730.00$ \\
\hline (20) & 70 & 90 & 34. & $3,105.00$ \\
\hline all meri & 71 & 90 & 34.50 & $3,105.00$ \\
\hline$\cdots$ & 72 & 30 & 40. & $3,600.00$ \\
\hline$\ldots \ldots \ldots \ldots$ & 73 & $\begin{array}{l}90 \\
60\end{array}$ & 39.50 & $3,555.00$ \\
\hline 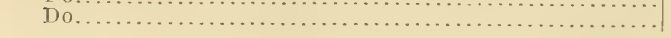 & 75 & 60 & $\begin{array}{l}38.50 \\
43.50\end{array}$ & $2,610.00$ \\
\hline
\end{tabular}


Details of sale of 6,100 dressed, dyed, and machined Pribilof Islands fur-seal skins at St. Louis, Mo., A pril 22, 1918-Continued.

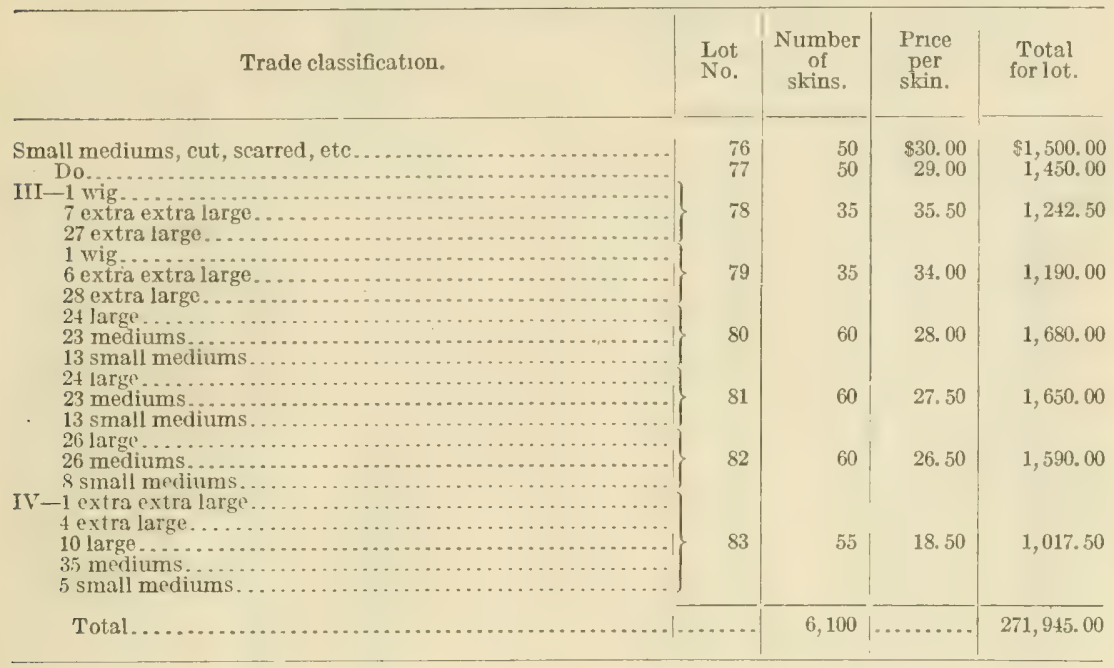

IX.

Details of sale of 2.ono dressed, dyed and machined Pribilof Islands fur-seal skins at St. Louis, October $7,1918$.

Trade classification.

Wigs. .

Extra extra large

Do.

Do.

Do

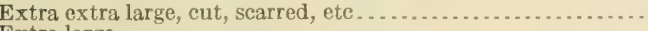

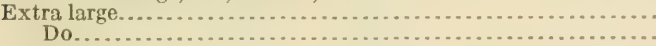

Do...

Do.

Do.

Extra large, cut, searred, etc.

Do......

Large...

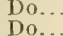

Do.

Do.

Do.

rge, cut, scarred, etc

Do..

Mediums.

Do

Do.

Mediums, cut, scarred, etc.

Small mediums.

$$
\text { Do.. }
$$

Small mediums, cut, searred, etco. 12 madia extra large.

12 medium.

12 large.................

Total

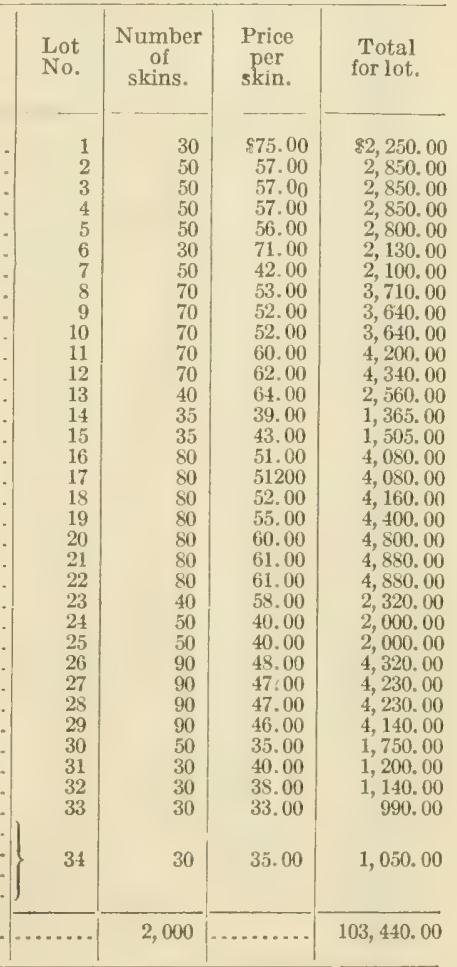


$\mathrm{X}$.

Detaits of sale of 10,103 dressed, dyed, and muchined Pribilof Islands fur-seul skins at st. Louis, Mo., A pril 28, 1919.

\begin{tabular}{|c|c|c|c|c|}
\hline Trade classification. & $\begin{array}{l}\text { Lot } \\
\text { No. }\end{array}$ & $\begin{array}{c}\text { Number } \\
\text { of } \\
\text { skins. }\end{array}$ & $\begin{array}{l}\text { Price } \\
\text { per } \\
\text { skin. }\end{array}$ & $\begin{array}{c}\text { Total } \\
\text { for lot. }\end{array}$ \\
\hline $\begin{array}{l}\text { Wigs... } \\
\text { Do. }\end{array}$ & 1 & 50 & $\$ 81.00$ & $\$ 4,050,00$ \\
\hline $\begin{array}{l}\text { Do. } \\
\text { Do. }\end{array}$ & 2 & 50 & 81.00 & $\begin{array}{r}\$, 000.00 \\
4,050.00\end{array}$ \\
\hline Do... & 3 & 50 & 80.00 & $4,000.00$ \\
\hline Do.. & $\begin{array}{l}4 \\
5\end{array}$ & $\begin{array}{l}50 \\
50\end{array}$ & 82.00 & $4,100.00$ \\
\hline Do.. & 6 & $\begin{array}{l}50 . \\
50\end{array}$ & $\begin{array}{l}81.00 \\
85.00\end{array}$ & $\begin{array}{l}4,050.00 \\
4,250.00\end{array}$ \\
\hline $\begin{array}{l}\text { Do.. } \\
\text { Do.. }\end{array}$ & 7 & 50 & 82.00 & $\begin{array}{l}4,250.00 \\
4,100.00\end{array}$ \\
\hline $\begin{array}{l}\text { Do.. } \\
\text { Do.. }\end{array}$ & 8 & 50 & 84.00 & $4,200.00$ \\
\hline Do.: & $\begin{array}{r}9 \\
10\end{array}$ & 50 & 84.00 & $4,200.00$ \\
\hline ............ & $\begin{array}{l}10 \\
11\end{array}$ & $\begin{array}{l}50 \\
50\end{array}$ & $\begin{array}{l}83.00 \\
88.00\end{array}$ & $\begin{array}{l}4,150.00 \\
4,400,00\end{array}$ \\
\hline Wigs, cut, scarred, etc. & 12 & 50 & 60.00 & $\begin{array}{l}4,400.00 \\
3,000.00\end{array}$ \\
\hline $\begin{array}{l}\text { Do } \ldots \ldots \ldots \ldots \\
\text { Do } \ldots \ldots \ldots\end{array}$ & 13 & 50 & 60.00 & $3,000,00$ \\
\hline Extra extra large... & $\begin{array}{l}14 \\
15\end{array}$ & $\begin{array}{l}50 \\
60\end{array}$ & $\begin{array}{r}62.00 \\
79.00\end{array}$ & $3,100.00$ \\
\hline Do................ & $\begin{array}{l}15 \\
16\end{array}$ & $\begin{array}{l}60 \\
60\end{array}$ & $\begin{array}{l}79.00 \\
77.00\end{array}$ & $\begin{array}{l}4,740.00 \\
4,620.00\end{array}$ \\
\hline (n...... & 17 & 60 & 78.00 & $\begin{array}{l}4,620.00 \\
4,680.00\end{array}$ \\
\hline Do $\ldots \ldots \ldots$. & 18 & 60 & 76.00 & $4,560.00$ \\
\hline $\begin{array}{l}\text { Do............... } \\
\text { Do ........ }\end{array}$ & 19 & 60 & 76.00 & $4,560.00$ \\
\hline $\begin{array}{l}\text { Do } \ldots . . . . . \\
\text { Do........... }\end{array}$ & 20 & 60 & 76.00 & $4,560.00$ \\
\hline $\begin{array}{l}\text { Do... } \\
\text { Do.. }\end{array}$ & 21 & 60 & 78.00 & $4,680.00$ \\
\hline $\begin{array}{l}\text { Do... } \\
\text { Do... }\end{array}$ & 22 & 60 & 79.00 & $4,740.00$ \\
\hline Do... & $\begin{array}{l}23 \\
24\end{array}$ & $\begin{array}{l}60 \\
60\end{array}$ & 77.50 & $4,650.00$ \\
\hline Do... & 25 & $\begin{array}{l}60 \\
60\end{array}$ & $\begin{array}{l}76.50 \\
80.00\end{array}$ & $4,590.00$ \\
\hline Do... & 26 & 60 & $\begin{array}{l}80.00 \\
76.00\end{array}$ & $\begin{array}{l}4,800.00 \\
4,560.00\end{array}$ \\
\hline $\begin{array}{l}\text { Do... } \\
\text { Do.. }\end{array}$ & 27 & 60 & 77.50 & $4,650.00$ \\
\hline $\begin{array}{l}\text { Do } \\
\text { Do } \ldots \ldots \ldots \ldots \ldots \ldots \ldots\end{array}$ & 28 & 60 & 75.00 & $4,500.00$ \\
\hline $\begin{array}{l}\text { Do } 0 \ldots \ldots \ldots \\
\text { Do }\end{array}$ & $\begin{array}{l}29 \\
30\end{array}$ & 60 & 77.00 & $4,620.00$ \\
\hline $\begin{array}{l}\text { Do... } \\
\text { Do... }\end{array}$ & $\begin{array}{l}30 \\
31\end{array}$ & $\begin{array}{l}60 \\
60\end{array}$ & 74.00 & $4,440.00$ \\
\hline Do... & $\begin{array}{l}01 \\
32\end{array}$ & $\begin{array}{l}60 \\
60\end{array}$ & $\begin{array}{l}77.00 \\
75.00\end{array}$ & $\begin{array}{l}4,620.00 \\
4,500.00\end{array}$ \\
\hline $\begin{array}{l}\text { Do... } \\
\text { Do... }\end{array}$ & 33 & 60 & 75.00 & $4,500.00$ \\
\hline $\begin{array}{l}\text { Do.... } \\
\text { Do... }\end{array}$ & 34 & 60 & 76.00 & $4,560.00$ \\
\hline Do.... & $\begin{array}{l}35 \\
36\end{array}$ & 60 & 78.00 & $4,680.00$ \\
\hline Do $\begin{array}{l}\text { Do... } \\
\text { Do }\end{array}$ & $\begin{array}{l}30 \\
37\end{array}$ & $\begin{array}{l}60 \\
60\end{array}$ & $\begin{array}{l}75.00 \\
75.00\end{array}$ & $\begin{array}{l}4,500.00 \\
4,500.00\end{array}$ \\
\hline $\begin{array}{l}\text { Do.... } \\
\text { Do.... }\end{array}$ & 38 & 60 & 75.00 & $4,500.00$ \\
\hline Do.... & $\begin{array}{l}39 \\
40\end{array}$ & 60 & 74.00 & $4,440.00$ \\
\hline Extra extra large; cut, searr & $\begin{array}{l}40 \\
41\end{array}$ & $\begin{array}{l}60 \\
60\end{array}$ & $\begin{array}{l}79.00 \\
59.00\end{array}$ & 00 \\
\hline Do.................... & 42 & 60 & $\begin{array}{l}59.00 \\
57.00\end{array}$ & $\begin{array}{l}3,510.00 \\
3,420.00\end{array}$ \\
\hline an. & 43 & 60 & 58.00 & $3,480.00$ \\
\hline 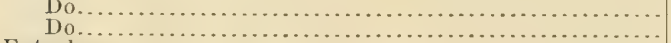 & 44 & 60 & 61.00 & $3,660.00$ \\
\hline Extra large.. & $\begin{array}{l}45 \\
46\end{array}$ & $\begin{array}{l}50 \\
70\end{array}$ & $\begin{array}{l}62.00 \\
73.00\end{array}$ & 00 \\
\hline $\mathrm{D}$ & 47 & 70 & 74.00 & $\begin{array}{l}5,110.00 \\
5,180.00\end{array}$ \\
\hline D & 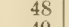 & 70 & 76.00 & $5,320.60$ \\
\hline D & 49 & 70 & 74. & .00 \\
\hline T & 50 & 70 & 74.50 & $5,215.00$ \\
\hline I) & 51 & 70 & 75. & 0.00 \\
\hline 1) & 52 & 70 & 76. & $5,320.00$ \\
\hline $\mathrm{D}$ & 5 & 70 & 79 & 5,5 \\
\hline $\begin{array}{l}\mathrm{D} \\
\mathrm{D}\end{array}$ & 54 & 70 & 77. & 0.00 \\
\hline & 55 & 70 & 78.00 & $5,460.00$ \\
\hline $\begin{array}{l}\text { D } \\
\text { D }\end{array}$ & 56 & 70 & 75. & .00 \\
\hline & 57 & 70 & 77.00 & .00 \\
\hline & 58 & 70 & 78.00 & $5,460.00$ \\
\hline${ }_{\cdots} \ldots \ldots$ & 59 & 70 & 77 & .00 \\
\hline & 60 & 70 & & $5,460.00$ \\
\hline & 61 & 50 & 77.0 & $3,850.00$ \\
\hline t, scarred, ete... & 62 & 45 & 77. & $3,465.00$ \\
\hline 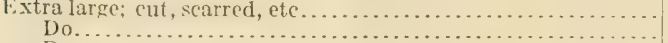 & 63 & 70 & 63. & $4,410.00$ \\
\hline$\ldots \ldots \ldots$ & 64 & 70 & 65 & $4,550.00$ \\
\hline $\begin{array}{l}10 \\
\text { rge }\end{array}$ & 65 & 70 & 65.00 & $4,550.00$ \\
\hline$\stackrel{\mathrm{rgc}}{\mathrm{r}}$ & 66 & 80 & 74.00 & $5,920.00$ \\
\hline $\mathrm{D}$ & 67 & 80 & 74 & $5,920.00$ \\
\hline & 68 & $\begin{array}{l}80 \\
80\end{array}$ & $\begin{array}{l}80.00 \\
77.00\end{array}$ & $\begin{array}{l}6,400.00 \\
6,160.00\end{array}$ \\
\hline 1) & 70 & 80 & 77.00 & $6,160.00$ \\
\hline & 71 & 80 & 77.00 & $6,160.00$ \\
\hline ......... & 72 & 80 & 77.50 & $6,200.00$ \\
\hline & 73 & 80 & 78.50 & $6,280.00$ \\
\hline 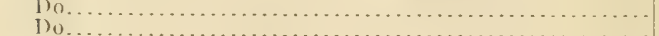 & 74 & 80 & 79.50 & $6,360.00$ \\
\hline D & 75 & $\begin{array}{l}80 \\
80\end{array}$ & $\begin{array}{l}78.00 \\
80.00\end{array}$ & $\begin{array}{l}6,210.00 \\
6,400.00\end{array}$ \\
\hline I0.. & 77 & 80 & 83.00 & $6,640.00$ \\
\hline
\end{tabular}


Details of sale of 10,102 dressed, dyed, and machined Pribilof Islands fur-seal skins at St. Louis, Mo., April 28, 1919-Continued.

\begin{tabular}{|c|c|c|c|c|}
\hline Trado classification. & $\begin{array}{l}\text { Lot } \\
\text { No. }\end{array}$ & $\begin{array}{c}\text { Number } \\
\text { of } \\
\text { skins. }\end{array}$ & $\begin{array}{c}\text { Price } \\
\text { per } \\
\text { skin. }\end{array}$ & $\begin{array}{l}\text { Total } \\
\text { lor lot. }\end{array}$ \\
\hline 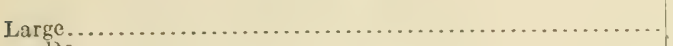 & is & 80 & $\operatorname{si1.00}$ & $\$ 6,480.00$ \\
\hline 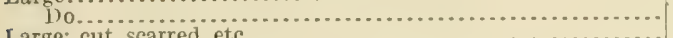 & 79 & 50 & 79.00 & $3,950.00$ \\
\hline Large; cut, scarred, etc. & 80 & 80 & $\div 0.00$ & $4,800.00$ \\
\hline Do & 81 & 80 & bit. 00 & $5,120.00$ \\
\hline Mediums... & 82 & 80 & 65.00 & $5,200.00$ \\
\hline $\begin{array}{r}\text { Mediums. } \\
\text { Do }\end{array}$ & 83 & 90 & 69.00 & $6,210.00$ \\
\hline $\begin{array}{l}\text { Do } \\
\text { Do }{ }_{1} \ldots \ldots\end{array}$ & 84 & 90 & 70.50 & $6,345.00$ \\
\hline 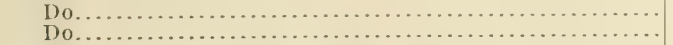 & 85 & 90 & 70.00 & $6,300.00$ \\
\hline 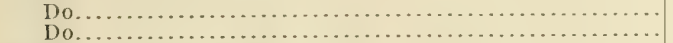 & 86 & 90 & 75.00 & $6,750.00$ \\
\hline 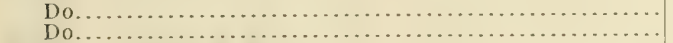 & 87 & 90 & 73.00 & $6,570.00$ \\
\hline 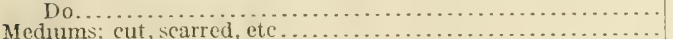 & 88 & 90 & 73.00 & $6,570.00$ \\
\hline 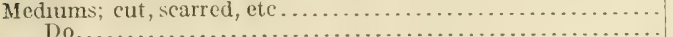 & 89 & 90 & 56.00 & $5,040.00$ \\
\hline 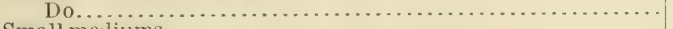 & 90 & 90 & $5 \$ .00$ & $5,220.00$ \\
\hline$\ldots \ldots \ldots \ldots \ldots \ldots \ldots \ldots \ldots \ldots \ldots \ldots$ & 91 & 50 & 60.00 & $3,000.00$ \\
\hline 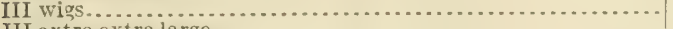 & 92 & 50 & 51.00 & $2,550.00$ \\
\hline 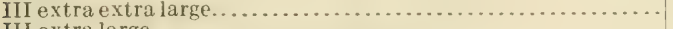 & 93 & 5.5 & 53.00 & $2,915.00$ \\
\hline 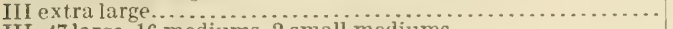 & 94 & 45 & 48. 60 & $2,160.00$ \\
\hline 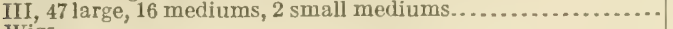 & 95 & 65 & 49.00 & 5.00 \\
\hline 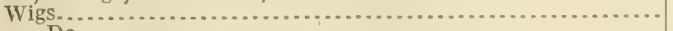 & 101 & 45 & 79.00 & $3,555.00$ \\
\hline 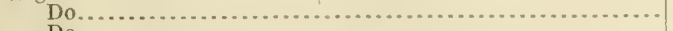 & 102 & 45 & 77.00 & $3,465.00$ \\
\hline 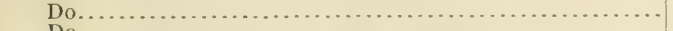 & 103 & 45 & 78.00 & $3,510.00$ \\
\hline 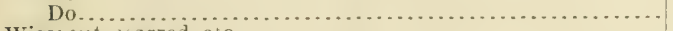 & 104 & 45 & 77.00 & 5.00 \\
\hline 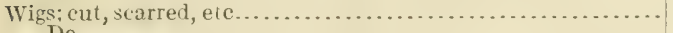 & 105 & 45 & 57.00 & 5.00 \\
\hline 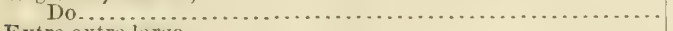 & 106 & 43 & 62.00 & 66. 00 \\
\hline$\ldots \ldots \ldots \ldots \ldots \ldots \ldots \ldots \ldots \ldots \ldots$ & 107 & 55 & 70.00 & .00 \\
\hline 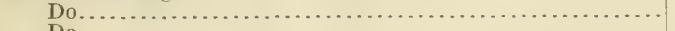 & 108 & 55 & 69.00 & 00 \\
\hline 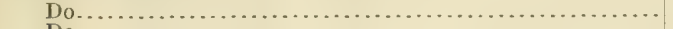 & 109 & 55 & 64.00 & 00 \\
\hline 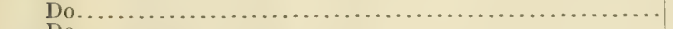 & 110 & 55 & 69. 110 & 00 \\
\hline 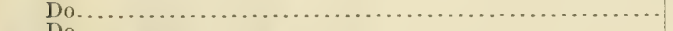 & 111 & 55 & 70.50 & 50 \\
\hline 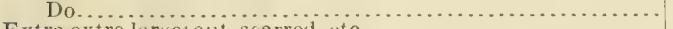 & 112 & 40 & 70.50 & 00 \\
\hline 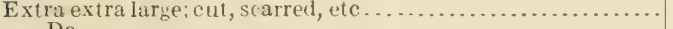 & 113 & 55 & 69.50 & 2.50 \\
\hline 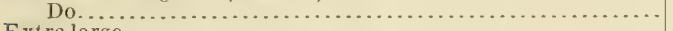 & 114 & 48 & 63.00 & 1.00 \\
\hline 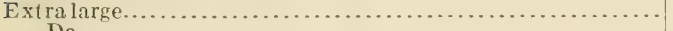 & 115 & 70 & 65.00 & .00 \\
\hline 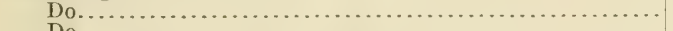 & 116 & 70 & 66.50 & .00 \\
\hline 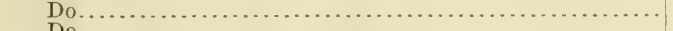 & 117 & 70 & 70.50 & 3.5 .00 \\
\hline$\ldots \ldots \ldots \ldots \ldots \ldots \ldots \ldots \ldots \ldots \ldots \ldots \ldots$, & 118 & 70 & 70.00 & .00 \\
\hline 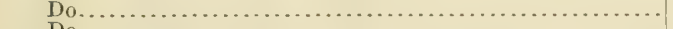 & 119 & 70 & 70.00 & .00 \\
\hline$\ldots \ldots \ldots \ldots$ & 120 & 70 & 70.00 & $4,900.00$ \\
\hline 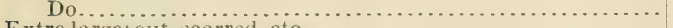 & 121 & 32 & 72.00 & $2,304.00$ \\
\hline 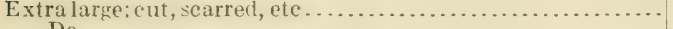 & 122 & 70 & 5.5 .50 & $3,885.00$ \\
\hline 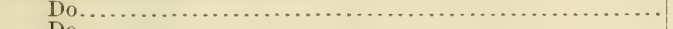 & 123 & 70 & 55.56 & $3,885.00$ \\
\hline 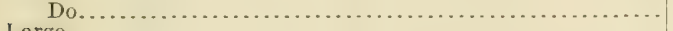 & 124 & 24 & 5.5 .50 & $1,332.00$ \\
\hline 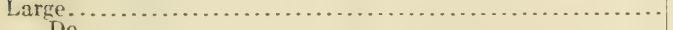 & 125 & 80 & 61.00 & $4,880.00$ \\
\hline 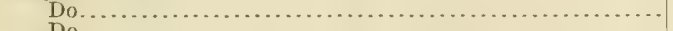 & 126 & 80 & 59.50 & $4,760.00$ \\
\hline 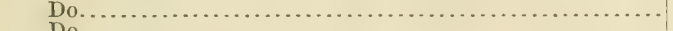 & 127 & 80 & 62.00 & .00 \\
\hline$\ldots \ldots \ldots \ldots \ldots, \ldots, \cdots, \ldots$, & 128 & 80 & 70. & .00 \\
\hline$\ldots \ldots \ldots \ldots$ & 129 & 80 & 69. & .00 \\
\hline 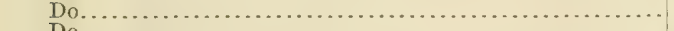 & 130 & 80 & 69 & .00 \\
\hline 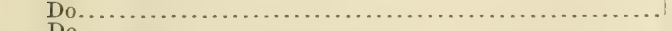 & 131 & 80 & 00 & 00 \\
\hline$\ldots \ldots \ldots \ldots \ldots \ldots \ldots$ & 132 & 80 & 64 & 00 \\
\hline$\ldots \ldots \ldots \ldots \ldots \ldots \ldots$ & 133 & 50 & 67. & 0.00 \\
\hline$\ldots \ldots \ldots \ldots \ldots \ldots \ldots$ & 134 & 45 & 70. & $3,150.00$ \\
\hline Large; cut, se & 135 & 80 & 5.5. & 0.00 \\
\hline Do.......... & 136 & 80 & 50 . & .00 \\
\hline Do............. & 137 & 80 & 50.50 & .00 \\
\hline Mediums.... & 138 & 90 & 60. & 0.00 \\
\hline Do... & 139 & 90 & 63. & .00 \\
\hline Do........ & 140 & 90 & ti2. & .00 \\
\hline 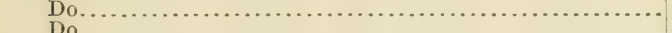 & 141 & 90 & 61.00 & 90.00 \\
\hline$\ldots \ldots \ldots \ldots \ldots \ldots$ & 142 & 90 & 58 & $5,265.00$ \\
\hline$\ldots \ldots \ldots \ldots+\cdots$ & 143 & 90 & 61. & $5,490.00$ \\
\hline Do... & 144 & 51 & 59.00 & $3,009.00$ \\
\hline$\ldots \ldots \ldots \ldots \ldots \ldots$ & 145 & 90 & 40.00 & $3,600,00$ \\
\hline (n) & 146 & 90 & 37.00 & $3,330.00$ \\
\hline Do.. & 147 & 90 & 39.00 & $3,510.00$ \\
\hline Do & 148 & 66 & 40.00 & $2,640.00$ \\
\hline all & 149 & 94 & 5i). 011 & 0.00 \\
\hline III 43 I & 150 & 74 & 28.00 & $2,072.00$ \\
\hline III, 43 wigs, 1 & 151 & 57 & 39.190 & $2,223.00$ \\
\hline III extra large & 152 & 48 & $4(1,(x)$ & $1,920.00$ \\
\hline III large.... & 153 & 79 & $30.00)$ & $2,370.00$ \\
\hline III mediums. & 154 & 87 & 27.50 & $2,392.50$ \\
\hline ........... & 155 & 85 & 27.50 & $2,337.50$ \\
\hline 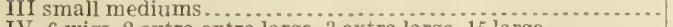 & 156 & 71 & $2 \cdot 3.00$ & $1,6,3.3 .(16)$ \\
\hline IV, 6 wigs, 2 extra extra large, 3 extra large, 15 large...... . . & 157 & 26 & 15. $(x)$ & 468.00 \\
\hline 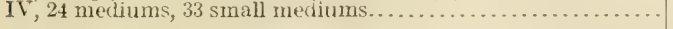 & 158 & 57 & 111. (H) & $570 .(10$ \\
\hline & & 10,102 & & $674,491.00$ \\
\hline
\end{tabular}


XI.

Details of sale of 9,055 dressed, dyed, and machined Pribilof Island fur-seal skins at S't. Louis, Mo., September 10, 1919.

\begin{tabular}{|c|c|c|c|c|}
\hline Trade classification. & $\begin{array}{l}\text { Lot } \\
\text { No. }\end{array}$ & $\begin{array}{c}\text { Number } \\
\text { of } \\
\text { skins. }\end{array}$ & $\begin{array}{l}\text { Price } \\
\text { pet } \\
\text { skin. }\end{array}$ & $\begin{array}{c}\text { Total } \\
\text { for lot. }\end{array}$ \\
\hline Wigs.. & 1 & 50 & 897.00 & $\$ 1,850.00$ \\
\hline Do.. & 2 & 50 & 101.00 & $5,050,00$ \\
\hline Do.. & 3 & 50 & 100.00 & $5,000,00$ \\
\hline Do.. & 4 & 50 & 101.00 & $5,050.00$ \\
\hline Do.. & 5 & 50 & 103. 00 & $5,150.00$ \\
\hline $\begin{array}{l}\text { Do.. } \\
\text { Do. }\end{array}$ & 6 & 50) & 105.00 & $5,250,00$ \\
\hline $\begin{array}{l}\text { Do.. } \\
\text { Do. }\end{array}$ & $\begin{array}{l}7 \\
8\end{array}$ & 50 & 102.00 & $5,100,00$ \\
\hline Do.. & $\begin{array}{l}8 \\
9\end{array}$ & 50 & 101.00 & $5,200.00$ \\
\hline Do.. & $\begin{array}{r}9 \\
10\end{array}$ & 50 & 106.00 & $5,300,00$ \\
\hline $\begin{array}{l}\text { Do.. } \\
\text { Do.. }\end{array}$ & $\begin{array}{l}10 \\
11\end{array}$ & 50. & 105.00 & $5,250.00$ \\
\hline Do... & 11 & 50 & 115.00 & $5,750.00$ \\
\hline $\begin{array}{l}\text { Do.... } \\
\text { vire: }\end{array}$ & 12 & 35 & 107.50 & $3,762.50$ \\
\hline $\begin{array}{l}\text { Wigs; eut, } \\
\text { Do }\end{array}$ & 13 & 50 & 74.00 & $3,700,00$ \\
\hline Do....... & 14 & 50 & 77.00 & $3,850.00$ \\
\hline $\begin{array}{l}\text { Extra extra large.. } \\
\text { Do............ }\end{array}$ & $\begin{array}{l}15 \\
16\end{array}$ & 60 & 105.00 & $6,300.00$ \\
\hline $\begin{array}{l}\text { Do.... } \\
\text { Do... }\end{array}$ & $\begin{array}{l}16 \\
17\end{array}$ & $\begin{array}{l}60 \\
60\end{array}$ & $\begin{array}{l}104.00 \\
10.00\end{array}$ & $\begin{array}{l}6,240.00 \\
6,300,00\end{array}$ \\
\hline Do.... & 18 & 60 & $107.5 n$ & $6,450.00$ \\
\hline Do.... & 19 & 60 & 104.00 & $6,210.00$ \\
\hline Do... & 20 & 60 & 110.00 & $6,600.00$ \\
\hline Do.. & 21 & 60 & 109.00 & $6,540.00$ \\
\hline Do.. & 22 & 60 & 107.00 & $6,+20,00$ \\
\hline Do... & $2 ; 3$ & 60 & 107.00 & $6,420.00$ \\
\hline Do.... & 24 & 60 & 106.010 & $6,360.00$ \\
\hline Do... & 25 & 60 & 109.00 & $6,540.00$ \\
\hline $\begin{array}{l}\text { Do.... } \\
\text { Do }\end{array}$ & 26 & 60 & 106.00 & $6,360.00$ \\
\hline 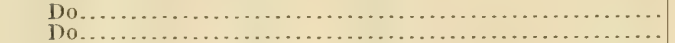 & 27 & 60 & 110.00 & $6,600.00$ \\
\hline $\begin{array}{l}\text { Do..... } \\
\text { Do.... }\end{array}$ & 29 & 60 & $10 \mathrm{~s} .00$ & $6,480.00$ \\
\hline $\begin{array}{l}\text { Do..... } \\
\text { Do.... }\end{array}$ & 29 & 60 & 111.00 & $6,660.00$ \\
\hline ......... & 30 & 60 & 111.00 & $6,660.00$ \\
\hline Do.... & 31 & 60 & 112.00 & $6,720.00$ \\
\hline $\begin{array}{l}\text { Do.... } \\
\text { Do.... }\end{array}$ & 32 & 60 & 111.00 & $6,660,00$ \\
\hline $\begin{array}{l}\text { Do.... } \\
\text { Do.... }\end{array}$ & $\begin{array}{l}33 \\
31\end{array}$ & 60 & 110.00 & $6,600.00$ \\
\hline $\begin{array}{l}\text { Do..... } \\
\text { Do.... }\end{array}$ & $\begin{array}{l}34 \\
35\end{array}$ & 60 & $112.01)$ & $6,720.00$ \\
\hline $\begin{array}{l}\text { Do....... } \\
\text { Extra extra }\end{array}$ & $\begin{array}{l}35 \\
36\end{array}$ & 60 & 115. 00 & $6,900.00$ \\
\hline 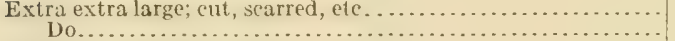 & $\begin{array}{l}36 \\
37\end{array}$ & 60 & $97 .(1)$ & $5, \$ 20.00$ \\
\hline Do.... & $\begin{array}{l}37 \\
38\end{array}$ & $\begin{array}{l}60 \\
60\end{array}$ & 95.00 & \\
\hline Extralar & 39 & $\begin{array}{l}60 \\
70\end{array}$ & $\begin{array}{r}95.00 \\
100.00\end{array}$ & $\begin{array}{l}5,700.00 \\
7,000.00\end{array}$ \\
\hline Do.. & 40 & 70 & 99.00 & $6,930.00$ \\
\hline Do.. & 41 & 70 & 95.00 & $6,560.00$ \\
\hline Do.. & $4:$ & 70 & 101.00 & $7,070.00$ \\
\hline Do.. & 43 & 70 & 102.00 & $7,140.00$ \\
\hline Do.. & 44 & 70 & 99.00 & $6,930.00$ \\
\hline Do & 45 & 70 & 96.00 & $6,720.00$ \\
\hline Do.. & 41 & 70 & 104.00 & $7,280.00$ \\
\hline Do.. & 47 & 70 & 99.00 & $6,930.00$ \\
\hline Do & $4 \mathrm{~s}$ & 70 & 99.00 & $6,930,00$ \\
\hline Do... & 49 & 70 & 101.00 & $7,070.00$ \\
\hline Do... & 50 & 70 & 104.00 & $7,250.00$ \\
\hline Do & 51 & 70 & 101.00 & $7,070.00$ \\
\hline Do. & 5 & 70 & 109.00 & $7,630.00$ \\
\hline Do.. & 53 & 70 & 104.50 & $7,315,00$ \\
\hline De & 5 & 70 & 10.5 .00 & $7,350.00$ \\
\hline Do..... & 55 & 70 & 107. 00 & $7,190.00$ \\
\hline Extra large; cut, scarred, & 56 & 70 & 95.00 & $6,650.00$ \\
\hline Do..................... & 57 & 70 & $\$ 7.00$ & $6,090.00$ \\
\hline Do... & 55 & 50 & $9 \bar{s} .00$ & $4,750.00$ \\
\hline Large. . & 59 & so & 101.00 & $8,080.00$ \\
\hline D & 60 & 80 & 100.00 & $8,000.00$ \\
\hline & 61 & 80 & 101.00 & $8,080.00$ \\
\hline Do... & 62 & 80 & 100.00 & $8,000.00$ \\
\hline Do & 63 & so & 100. 09 & $8,000.00$ \\
\hline Dc & 64 & 80 & 103. 01$)$ & $8,240.00$ \\
\hline & 65 & so & 100.00 & $8,000.00$ \\
\hline Do... & 66 & 80 & 100.00 & $8,000.00$ \\
\hline Do... & 67 & 80 & 99.00 & $7,920.00$ \\
\hline $\mathrm{D}$ & $6 s$ & 80 & 105.00 & $8,400.03$ \\
\hline Do.. & 69 & so & 104.00 & $8,320.00$ \\
\hline Do.. & 70 & so & 102.00 & $8,160,00$ \\
\hline Do.. & 71 & 80 & 101.00 & $8,0 \times 0,00$ \\
\hline & 72 & so & 101.00 & $8,0 \leq 0.00$ \\
\hline …… & 73 & so & 100.00 & $8,060.00$ \\
\hline (2) & 74 & $x_{0}$ & 100.00 & $8,0(0,00$ \\
\hline (n. & 75 & 80 & 1111.00 & $8,080.00$ \\
\hline & & & & $8,240.90$ \\
\hline
\end{tabular}


Detail of sale of 9,05.5 pressed, dyed, and machined Pribilof Island fur-seal slins at St. Louis, Mo., September 10, 1919-Continued.

\begin{tabular}{|c|c|c|c|c|}
\hline Trade classification. & $\begin{array}{l}\text { Lot } \\
\text { No. }\end{array}$ & $\begin{array}{c}\text { Number } \\
\text { of } \\
\text { skins. }\end{array}$ & $\begin{array}{c}\text { Price } \\
\text { per } \\
\text { skin. }\end{array}$ & $\begin{array}{l}\text { Total } \\
\text { for lot. }\end{array}$ \\
\hline \multicolumn{5}{|l|}{ 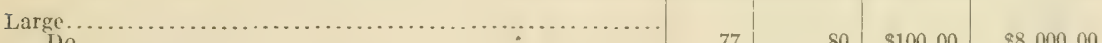 } \\
\hline $\begin{array}{l}D_{0} \\
D_{0}\end{array}$ & 77 & 80 & $\$ 100.00$ & $88,000.00$ \\
\hline 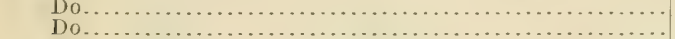 & 78 & 80 & 102.50 & 0.00 \\
\hline 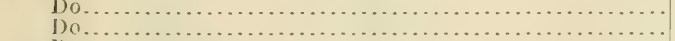 & 79 & 80 & 101.00 & 0.00 \\
\hline Do & 80 & 80 & 102,00 & 0.00 \\
\hline 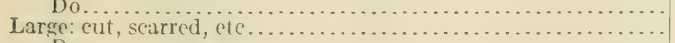 & 81 & 80 & 102.00 & 50.00 \\
\hline 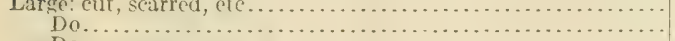 & 82 & 80 & 79.00 & 20.00 \\
\hline 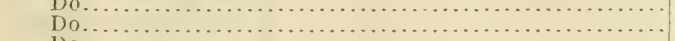 & 83 & 80 & 81.00 & 30.00 \\
\hline & 8 & 80 & 80.00 & 0.00 \\
\hline 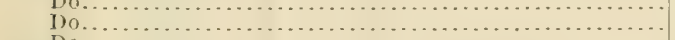 & si & 80 & 78.00 & 10.00 \\
\hline (n. & 8 & 80 & 80.00 & 0.00 \\
\hline 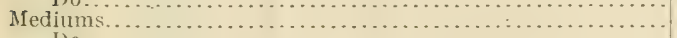 & $\begin{array}{l}87 \\
88\end{array}$ & $\begin{array}{l}80 \\
90\end{array}$ & 82.00 & 3000 \\
\hline 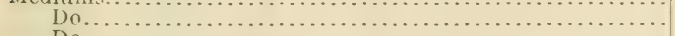 & $\begin{array}{l}58 \\
89\end{array}$ & $\begin{array}{l}90 \\
90\end{array}$ & 10 & 0.00 \\
\hline Do................. & 90 & $\begin{array}{l}90 \\
90\end{array}$ & $\begin{array}{l}8.00 \\
85.00\end{array}$ & .00 \\
\hline 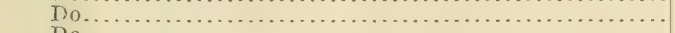 & 91 & $\begin{array}{l}90 \\
90\end{array}$ & 82.00 & 0.00 \\
\hline 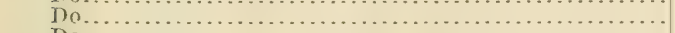 & 92 & 90 & 82.50 & 30.00 \\
\hline 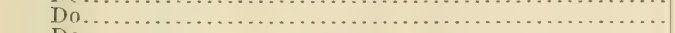 & 93 & 90 & 82.00 & $\begin{array}{l}5.00 \\
0.00\end{array}$ \\
\hline$D C \ldots \ldots \ldots \ldots$ & 94 & $\begin{array}{l}90 \\
90\end{array}$ & 82.00 & $\begin{array}{l}0.00 \\
0.00\end{array}$ \\
\hline$\ldots \ldots$ & 95 & 90 & 83.00 & $\begin{array}{l}0.00 \\
0.00\end{array}$ \\
\hline no... & 96 & 90 & 83.00 & $\begin{array}{l}0.00 \\
0.00\end{array}$ \\
\hline$\ldots \ldots \ldots \ldots \ldots \ldots$ & 97 & 90 & 83.00 & $\begin{array}{l}0.00 \\
0.00\end{array}$ \\
\hline Do............. & 98 & 90 & 83.50 & $\begin{array}{l}0.00 \\
5.00\end{array}$ \\
\hline 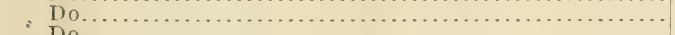 & 99 & 90 & 83.00 & .00 \\
\hline 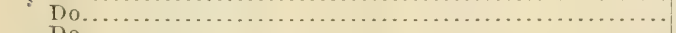 & 100 & 90 & 87.00 & .00 \\
\hline 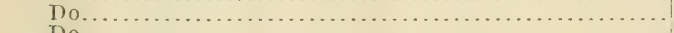 & 101 & 90 & 85.00 & .00 \\
\hline 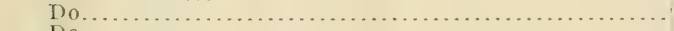 & 102 & 90 & 83.00 & 00 \\
\hline . . . & 103 & 90 & 83.00 & 00 \\
\hline$\ldots \ldots \ldots \ldots \ldots \ldots$ & 104 & 90 & 86.00 & $\begin{array}{l}00 \\
00\end{array}$ \\
\hline$\ldots \ldots \ldots \ldots \ldots \ldots \ldots$ & 105 & 90 & 84. & 00 \\
\hline$\ldots \ldots \ldots \ldots \ldots \ldots$ & 106 & 90 & 82.00 & 00 \\
\hline$\ldots \ldots \ldots \ldots \ldots \ldots \ldots$ & 107 & 90 & 85. & 00 \\
\hline$\ldots \ldots \ldots \ldots \ldots \ldots \ldots$ & 108 & 80 & & \\
\hline 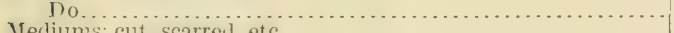 & 109 & 80 & & \\
\hline \multicolumn{5}{|l|}{ 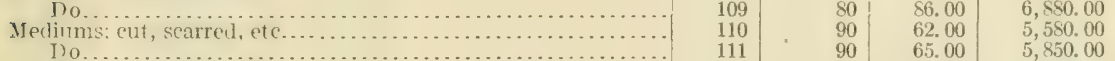 } \\
\hline $\begin{array}{l}\text { I) } 0 \ldots \\
\text { T) } 0 \ldots\end{array}$ & 111 & 90 & 65 & 50.00 \\
\hline 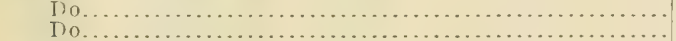 & 112 & 90 & 67 & $6,030.00$ \\
\hline 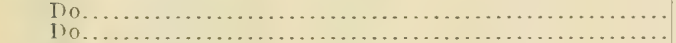 & 113 & 90 & & 00 \\
\hline 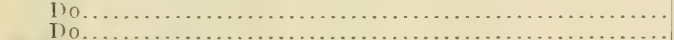 & 11 & 90 & & 00 \\
\hline 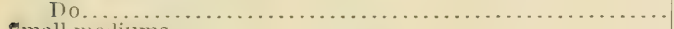 & 11 & 60 & & 00 \\
\hline \multicolumn{5}{|l|}{ 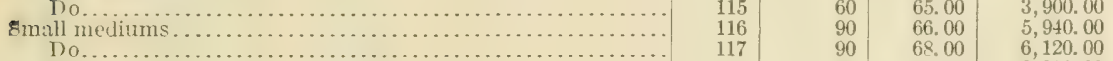 } \\
\hline $\begin{array}{l}\text { Do... } \\
\text { Do... }\end{array}$ & 11 & 90 & 68. & $6,120.00$ \\
\hline $\begin{array}{ll}\cdots \\
\cdots\end{array}$ & 11 & 90 & 69.00 & $6,210.00$ \\
\hline \multirow{7}{*}{\multicolumn{5}{|c|}{ 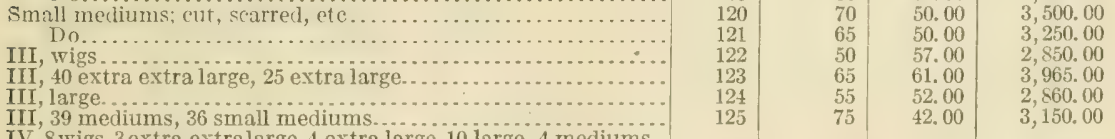 }} \\
\hline & & & & \\
\hline & & & & \\
\hline & & & & \\
\hline & & & & \\
\hline & & & & \\
\hline & & & & \\
\hline \multicolumn{5}{|l|}{ 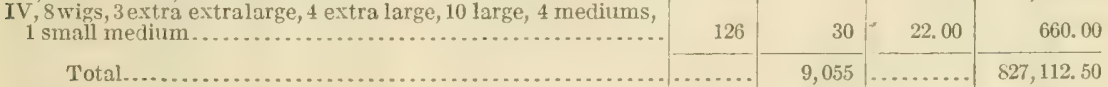 } \\
\hline & & 9,055 & & 827 , \\
\hline
\end{tabular}

\section{XII.}

Details of sale of 9,100 dressed. dyed, and machined Pribilof Islunds fur-seul slins at St. Louis, Mo., February 2, 1920.

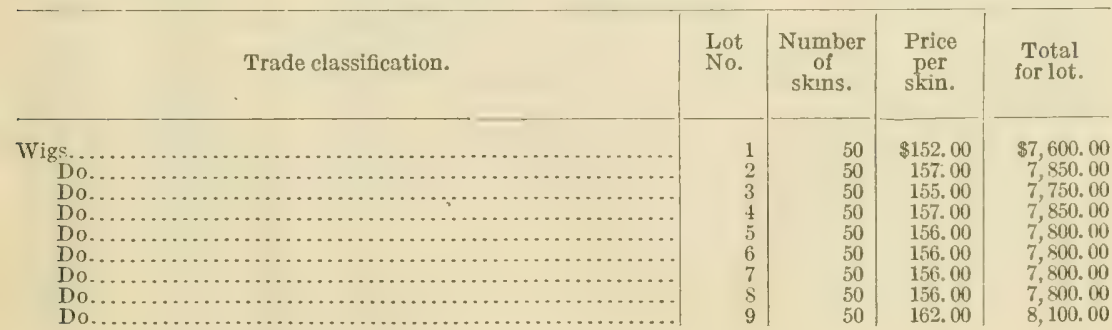


Details of sale of 9,100 dressed, dyed, and machined Pribilof Islands fur-seal skins at St. Louis, Mo., February 2, 1920-Continued.

\begin{tabular}{|c|c|c|c|c|}
\hline Trade classification. & $\begin{array}{l}\text { Lot } \\
\text { No. }\end{array}$ & $\begin{array}{c}\text { Number } \\
\text { of } \\
\text { skins. }\end{array}$ & $\begin{array}{l}\text { Price } \\
\text { per } \\
\text { skin. }\end{array}$ & $\begin{array}{l}\text { Total } \\
\text { for lot. }\end{array}$ \\
\hline \multirow{2}{*}{\multicolumn{5}{|c|}{$\begin{array}{r}\text { Wigs } \\
\text { Do }\end{array}$}} \\
\hline & 10 & 50 & $\$ 162.00$ & $\$ 8,100,00$ \\
\hline Do.... & 11 & 50 & 163.00 & $8,150.00$ \\
\hline Do.. & 12 & 50 & 167.00 & $8,350.00$ \\
\hline Do.. & 13 & 50 & 169.00 & $8,450.00$ \\
\hline Do... & 14 & 50 & 166.00 & $8,300.00$ \\
\hline Do... & 15 & 50 & 169.00 & $8,450.00$ \\
\hline $\begin{array}{l}\text { igs: cut, } \\
\text { Do... }\end{array}$ & 16 & 50 & 115.00 & $5,750.00$ \\
\hline $\begin{array}{l}\text { Do... } \\
\text { Do... }\end{array}$ & 17 & 50 & 116.00 & $5,800,00$ \\
\hline & 18 & 50 & 115.00 & $5,750.00$ \\
\hline $\begin{array}{l}\text { Do... } \\
\text { Do... }\end{array}$ & 19 & $\begin{array}{l}50 \\
50\end{array}$ & 117.00 & $5,850.00$ \\
\hline Do... & $\begin{array}{l}20 \\
21\end{array}$ & $\begin{array}{l}50 \\
50\end{array}$ & $\begin{array}{l}120.00 \\
118.00\end{array}$ & $\begin{array}{l}6,000.00 \\
5,900.00\end{array}$ \\
\hline xtra ext & 22 & 60 & 167.00 & $10,020.00$ \\
\hline Do... & 23 & 60 & 170.00 & $10,200.00$ \\
\hline Do.. & 24 & 60 & 167.00 & $10,020.00$ \\
\hline Do... & 25 & 60 & 166.00 & $9,960.00$ \\
\hline Do... & 26 & 60 & 166.00 & $9,960.00$ \\
\hline Do... & 27 & 60 & 171. 00 & $10,260.00$ \\
\hline $\begin{array}{l}\text { Do... } \\
\text { Do... }\end{array}$ & 28 & 60 & 172.00 & $10,320.00$ \\
\hline Do... & 29 & 60 & 170.00 & $10,200.00$ \\
\hline $\begin{array}{l}\text { Do... } \\
\text { Do. }\end{array}$ & 30 & 60 & 170.00 & $10,200.00$ \\
\hline $\begin{array}{l}\text { Do... } \\
\text { Do.. }\end{array}$ & 31 & 60 & . 171.00 & $10,260.00$ \\
\hline Do & 32 & 60 & 168.00 & $10,080.00$ \\
\hline Do & 33 & 60 & 167.00 & $10,020.00$ \\
\hline Do.. & 31 & 60 & 174.00 & $10,440.00$ \\
\hline Do & 35 & 60 & 170.00 & $10,200.00$ \\
\hline $\begin{array}{l}\text { Do } \\
\text { Do }\end{array}$ & 36 & 60 & 173.00 & $10,380.00$ \\
\hline$\ldots$ & 37 & 60 & 171.00 & $10,260.00$ \\
\hline Do & 38 & 60 & 169.00 & $10,140.00$ \\
\hline $\begin{array}{l}\text { Do } \\
\text { Dr }\end{array}$ & 39 & 60 & 165.00 & $10,0 \leq 0.00$ \\
\hline & 40 & 60 & 172.00 & $10,320.00$ \\
\hline & 41 & 60 & 172.00 & $10,320.00$ \\
\hline $\mathrm{D}$ & 42 & 60 & 172.00 & $10,320.00$ \\
\hline & 43 & 60 & 171.00 & $10,260,00$ \\
\hline Do.. & 44 & 60 & 171.00 & $10,260.00$ \\
\hline Extra ext & 45 & 60 & 124.00 & $7,440.00$ \\
\hline Do.. & 46 & 60 & 124.00 & $7,440.00$ \\
\hline Do... & 47 & 60 & 131.00 & $7,860.00$ \\
\hline & 48 & 60 & 127. 00 & $7,620.00$ \\
\hline Extra extra large; cut, & 49 & 60 & 130.00 & $7,800.00$ \\
\hline Do.................. & 50 & 60 & 127.00 & $7,620.00$ \\
\hline & 51 & 60 & 131.00 & $7,860.00$ \\
\hline I) & 52 & 6 & 130.00 & $7,800.00$ \\
\hline D & 53 & 60 & 131.00 & $7,860.00$ \\
\hline & 54 & 60 & 132.00 & $7,920.00$ \\
\hline xtra & 55 & 70 & 161.00 & $11,270.00$ \\
\hline & 56 & 70 & 169.00 & $11,830.00$ \\
\hline & 57 & 70 & 173.00 & $12,110.00$ \\
\hline & 58 & 70 & 172.00 & $12,010.00$ \\
\hline & 59 & 70 & 170.00 & $11,900.00$ \\
\hline & 60 & 70 & 174.00 & $12,180,00$ \\
\hline$\cdots$ & 61 & 70 & 171.00 & $11,970.00$ \\
\hline & 6 & 70 & 173.00 & $12,110.00$ \\
\hline $\mathrm{D}$ & 63 & 70 & 168.00 & $11,760.00$ \\
\hline D & 64 & 70 & 169.00 & $11,830.00$ \\
\hline & 6 & 70 & 170.00 & $11,900.00$ \\
\hline & 66 & 70 & 168.00 & $11,760.00$ \\
\hline & 67 & 70 & 168. & $11,760.00$ \\
\hline ... & 68 & 70 & 168.50 & $11,795.00$ \\
\hline ... & 6 & 70 & 177.00 & $12,390.00$ \\
\hline Extra & 7 & 70 & 134.00 & $9,380.00$ \\
\hline & 7 & 70 & 130.00 & $9,100.00$ \\
\hline$\cdots$ & 72 & 70 & 128.00 & $8,960.00$ \\
\hline & 73 & 70 & 127.00 & $8,890.00$ \\
\hline ...... & 74 & 70 & -129.00 & $9,030,00$ \\
\hline …... & 75 & 70 & 130.00 & $9,100.00$ \\
\hline rite & 76 & 80 & 160.00 & $12,800.00$ \\
\hline ... & 77 & 80 & 157.00 & $12,560.00$ \\
\hline ... & 78 & 80 & 164.00 & $13,120.00$ \\
\hline & 79 & 80 & 162.00 & $12,960.00$ \\
\hline ... & 80 & so & 162.00 & $12,960.00$ \\
\hline I & 81 & 80 & 161.00 & $12,880.00$ \\
\hline & 82 & 80 & 161.00 & $12,880.00$ \\
\hline I & 83 & 80 & 160.00 & $12,800.00$ \\
\hline$\cdots$ & 84 & 80 & 159.00 & $12,720.00$ \\
\hline .... & 85 & 80 & 160.00 & $12,800.00$ \\
\hline 170 & 86 & 80 & 161.00 & $12,880.00$ \\
\hline Do & 87 & 80 & 162.00 & $12,960.00$ \\
\hline
\end{tabular}


Details of sale of 9,100 dressed. dyed, and mactined Pribilof Island fur seal skins it Sit. Louis, Mo., February 2, 1920-Continued.

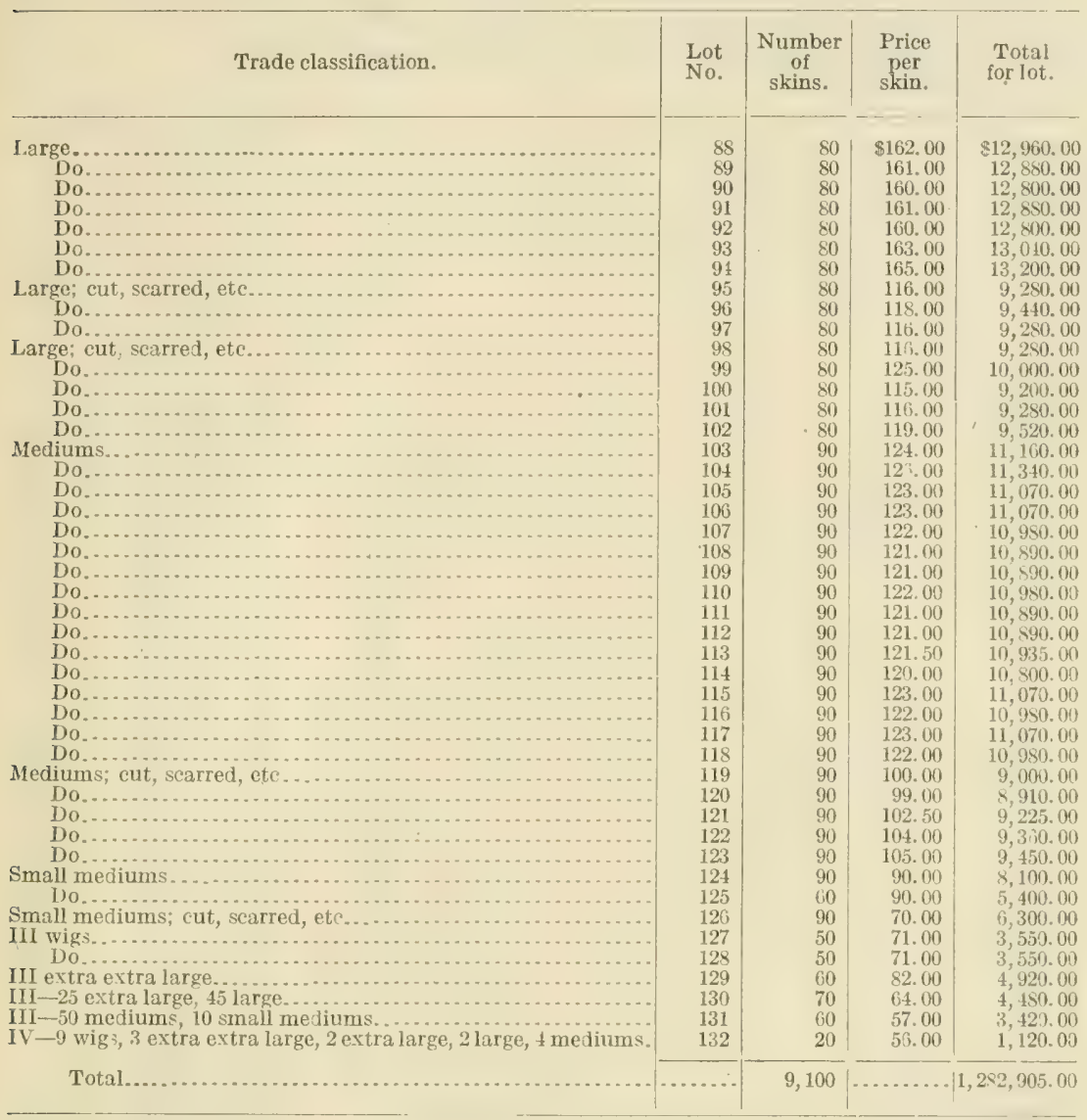

\section{XIII}

ST. Louis, December 11, 1919.

Mr. H. M. SмrTh,

Commissioner, Department of Commerce,

Bureau of Fisheries, Washington, D. C.

Dear Sir: Please refer to your letter dated November 13 bearing upon previous exchange of correspondence between our respective offices in regard to the so-called food skins remaining unsold after the April, 1919, sale.

With reference to the spoiled skins, there being 17 of these out of the 31 , the remain der of which are samples of work of our dressing and dyeing department at different periods, which we desire to obtain for exhibition, and on which we ofter a price of $\$ 80$ per slin.

This figure has been arrivel at on the hasis of the purchase price these stins hrmuht at the last sale, which basis we think is more fair.

Kindly arl vise us if this figure is satisfartory, and we will mail you our check to an: ar. Yours very truly,

Funsten Bros. \& Co. 
Messrs. Funsten Bros. \& Co., St. Louis, Mo.

December 20, 1919.

Gentlemen: In reply to your letter (BG-MK) of December 11, 1919, in regard to the 31 food skins, 17 of which were spoiled, and the others were prepared for exhibition or as samples of your work, your offer of $\$ 80$ per skin is accepted.

Very truly yours,

H. MI. Smith, Commissioner.

XIV.

Drtiits of s:le of 5,75: diessel, dyed, und machined Pribilof Islands fur-seal slins at st. Louis, Mo., May 10, 1920.

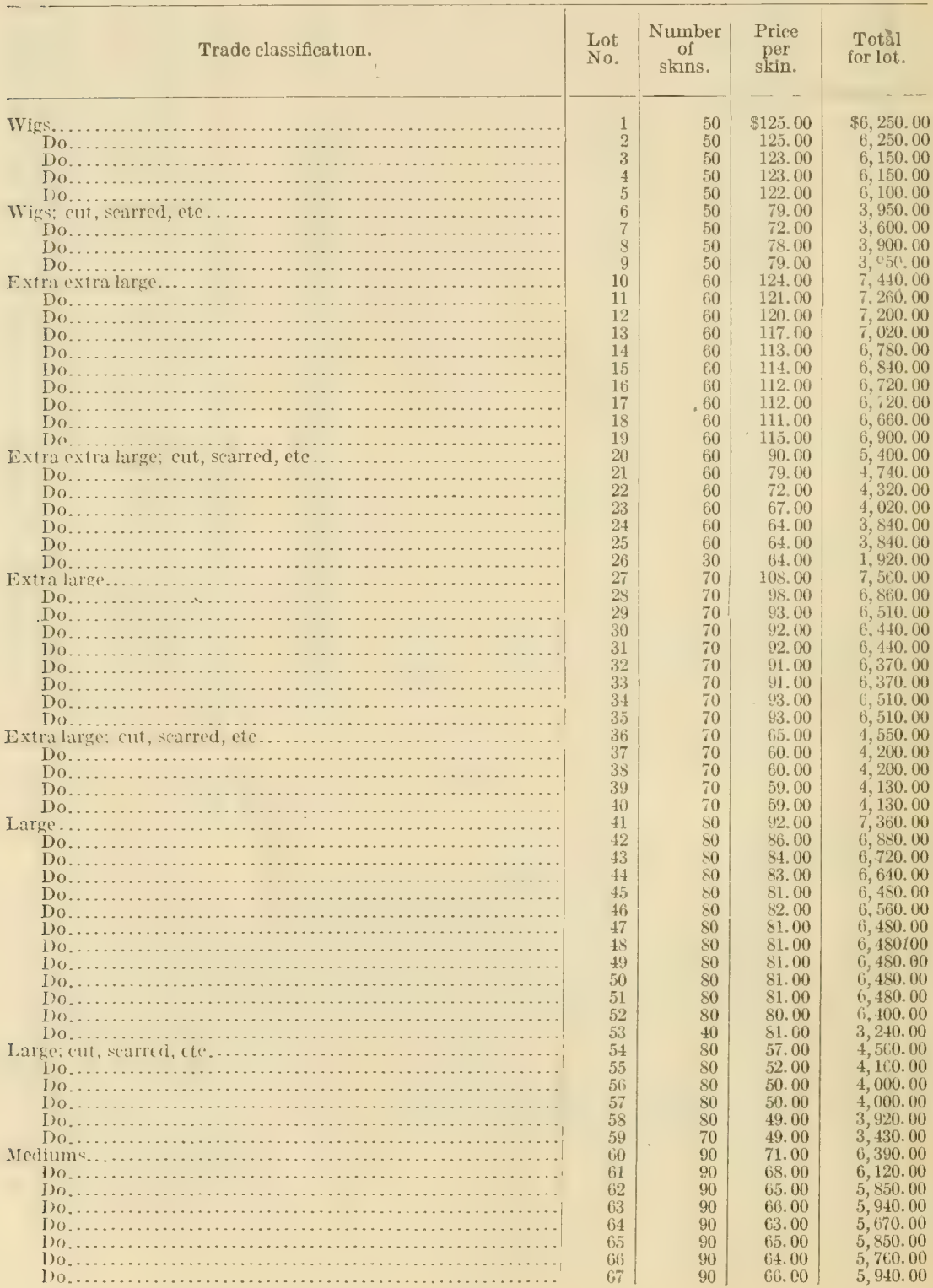


Detuils of sale of 5,752 dressed, dyed, and machined Pribilof Islands fur-seal stins at St. Louis, Mo., May 19, 1920-Continued.

\begin{tabular}{|c|c|c|c|c|}
\hline Trade classification. & $\begin{array}{l}\text { Lot } \\
\text { No. }\end{array}$ & $\begin{array}{c}\text { Number } \\
\text { of } \\
\text { skins. }\end{array}$ & $\begin{array}{l}\text { Price } \\
\text { per } \\
\text { skin. }\end{array}$ & $\begin{array}{l}\text { Total } \\
\text { for lot. }\end{array}$ \\
\hline 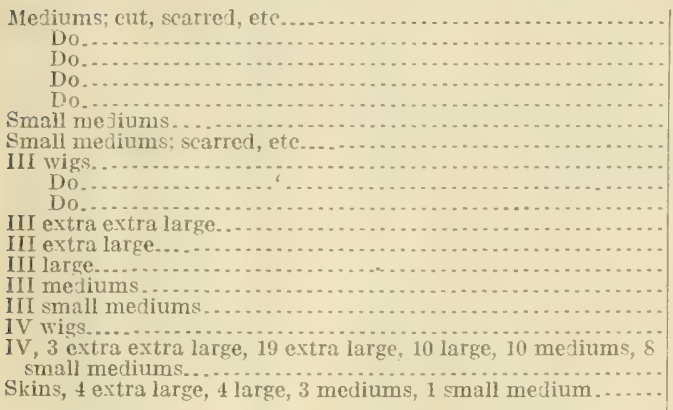 & $\begin{array}{l}6 S \\
70 \\
71 \\
72 \\
73 \\
74 \\
75 \\
76 \\
77 \\
78 \\
79 \\
80 \\
81 \\
82 \\
83\end{array}$ & $\begin{array}{l}90 \\
90 \\
90 \\
90 \\
90 \\
90 \\
70 \\
50 \\
50 \\
50 \\
50 \\
60 \\
60 \\
70 \\
30 \\
50 \\
50 \\
50 \\
12\end{array}$ & $\begin{array}{r}\$ 50.00 \\
45.00 \\
44.00 \\
41.00 \\
4.00 \\
55.00 \\
39.00 \\
40.00 \\
41.00 \\
41.00 \\
37.00 \\
37.00 \\
35.00 \\
27.00 \\
19.00 \\
10.00 \\
\\
21.00 \\
53.00\end{array}$ & $\begin{array}{r}84,500.00 \\
4,050.00 \\
3,9: 0.00 \\
3,930.00 \\
4,140.00 \\
4,950.00 \\
2,730.00 \\
2,000.00 \\
2,050.00 \\
2,050.00 \\
1,850.00 \\
2,220.00 \\
2,160.00 \\
1,820.00 \\
570.00 \\
500.00 \\
1,050.00 \\
636.00\end{array}$ \\
\hline 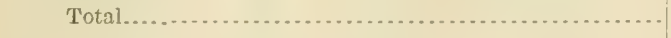 & & 5,752 & & $424,166.00$ \\
\hline
\end{tabular}

\section{XV.}

Details of sale of 10,120 dressed, dyed, and machined Pribilof Islands fur-seal stizus at St. Louis, Mo, on February 21, 1921.

Trade classification.

\begin{tabular}{|c|c|c|c|}
\hline $\begin{array}{l}\text { Lot } \\
\text { No. }\end{array}$ & $\begin{array}{c}\text { Number } \\
\text { of } \\
\text { skins. }\end{array}$ & $\begin{array}{l}\text { Price } \\
\text { per } \\
\text { skin. }\end{array}$ & $\begin{array}{l}\text { Total } \\
\text { for lot. }\end{array}$ \\
\hline 1 & 50 & sis(1, 101 & $\$ 3,000.00$ \\
\hline 2 & 50 & 58.169 & $2,900.00$ \\
\hline 3 & 50 & $\therefore 7.111$ & $1,850.00$ \\
\hline 4 & 60 & 585.161 & $3,360,00$ \\
\hline 5 & 60 & 61.1111 & $3,600.00$ \\
\hline 6 & 60 & 41.019 & $2,460.00$ \\
\hline 7 & 70 & 5.5 .010 & $3,850.00$ \\
\hline 8 & 70 & $51.01)$ & $3,741,1111$ \\
\hline 9 & 70 & 57.011 & $3, y: 41$ (स) \\
\hline 10 & 70 & 51. (n) & $3,7,40.1011$ \\
\hline 11 & 70 & 31.140 & $2,170.00$ \\
\hline 12 & so & 52,00 & $4,160.00$ \\
\hline $1: 3$ & 80 & $51.116)$ & $4,0 \mathrm{~N}(1,190$ \\
\hline 14 & 80 & 49.611 & $\therefore, 920,0101$ \\
\hline 15 & 80 & 4५. 100 & $\therefore .840 .00$ \\
\hline 16 & 80 & 49. 011 & 8.920 .00 \\
\hline 17 & 80 & $41 i, 001$ & $3,6 \times 0.00$ \\
\hline 18 & 80 & $46 .(10)$ & $3,650,00$ \\
\hline 19 & so & 17.01 & $3,760.00$ \\
\hline 20 & 80 & $4 \pi .0 .1$ & 3,76000 \\
\hline 21 & 80 & $27 . \pi(t)$ & $2,200.00$ \\
\hline 22 & 80 & 27.00 & $2,160(0)$ \\
\hline 23 & 80 & 2S. 31 & $2,2 \times 0,013$ \\
\hline 24 & 80 & 29.00 & $2,320.000$ \\
\hline 25 & 90 & 39.50 & $3,555.00$ \\
\hline 26 & 90 & 40. (111) & $3,000.011$ \\
\hline 27 & 90 & 42.010 & $3.7 \times(1.10$ \\
\hline $\begin{array}{l}28 \\
29\end{array}$ & $\begin{array}{l}30 \\
90\end{array}$ & 39.50 & $3,55,00$ \\
\hline $\begin{array}{l}29 \\
30\end{array}$ & $\begin{array}{l}90 \\
90\end{array}$ & $\begin{array}{l}40.00 \\
40.00\end{array}$ & $\begin{array}{l}3,6015)(10 \\
3,6010\end{array}$ \\
\hline 31 & 90 & 40. $5 !$ & $3,645.00$ \\
\hline 32 & 90 & 30.0101 & $2,700.00$ \\
\hline 33 & 90 & 29.001 & $2,610.00$ \\
\hline 34 & 90 & 29.110 & $2,+100$ \\
\hline 35 & 60 & : 26.0111 & $2,160.00$ \\
\hline 36 & 40 & $2-, 011$ & $1,0 \& 0.00$ \\
\hline 37 & 50 & $=1.001$ & $1,050.00$ \\
\hline 38 & 50 & 16.3 & $\$ 25.00$ \\
\hline 39 & 50 & 17. (11) & 5.50 .00 \\
\hline 40 & 50 & 13.54 & 975.00 \\
\hline 411 & 70 & 20.50 & $1,435.00$ \\
\hline
\end{tabular}

Wigs.....

Wigs, cut, scarred, etc

Extra extra large.

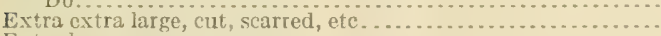

Extra large. . . . . . . . . . . . . . . .

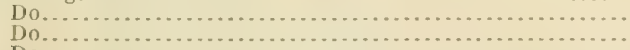

Extra large, cut, searred, ete. . . . Large.

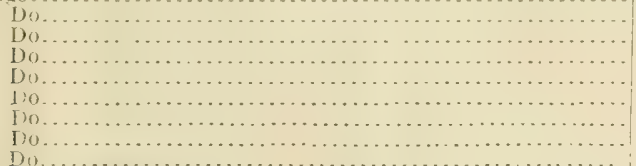

Do.

large: cut, surrerl, cte . . . .

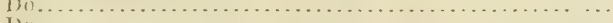

D).......

Medium.

De.........

Do. . . .

Do. . .

$\mathrm{D}(\mathrm{c}$.

Di)

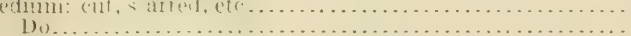

1)

smell, minjum

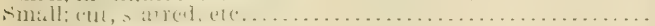

III 11 igs. .

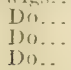

III, 45 extra exira large; 25 extra large 
Detaits of sale of 10,120 dresscd, dyed, and machined Pribilof Islands fur-seal skins at St. Louis, Mo., on February 21, 1921-Continued.

\begin{tabular}{|c|c|c|c|c|}
\hline Trade classification. & $\begin{array}{l}\text { Lot } \\
\text { No. }\end{array}$ & $\begin{array}{c}\text { Number } \\
\text { of } \\
\text { skins. }\end{array}$ & $\begin{array}{l}\text { Price } \\
\text { per } \\
\text { skin. }\end{array}$ & $\begin{array}{l}\text { Total } \\
\text { for lot. }\end{array}$ \\
\hline III large. . . . . . . . . . . . . & 12 & 80 & $\$ 19.00$ & $\$ 1,520,00$ \\
\hline III, 50 medium; 10 small medium. . . . . . . . . . . . & 43 & 50 & 20.00 & $1,200,00$ \\
\hline 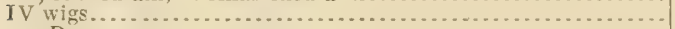 & 44 & 50 & 10.00 & 500.00 \\
\hline 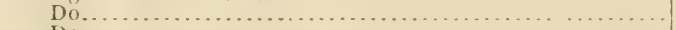 & 15 & 50 & 7.50 & 5.00 \\
\hline 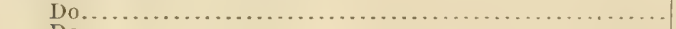 & $4 t i$ & 50 & 8. 00 & .00 \\
\hline 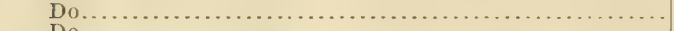 & 47 & 50 & 10.00 & 500.00 \\
\hline 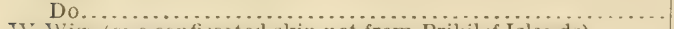 & $4 x$ & 50 & 9.50 & 5. $0(0)$ \\
\hline IT Wigs (nue confiseated skin not from Pribilof Islands) ...... & 49,50 & 50 & 9.00 & 0.00 \\
\hline 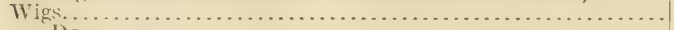 & 51 & 50 & 55.50 & $2,775.00$ \\
\hline 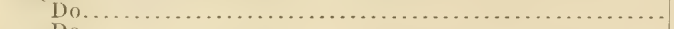 & 52 & 50 & 55. 00 & $2,750,00$ \\
\hline 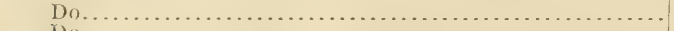 & 53 & 50 & 55.00 & $2,750.00$ \\
\hline 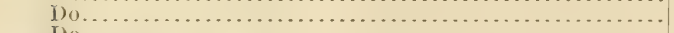 & 54 & 50 & 56.00 & $2,800.00$ \\
\hline 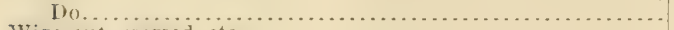 & 5.5 & 32 & 55.00 & $1,760.00$ \\
\hline 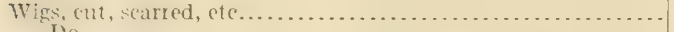 & 56 & 50 & 35.00 & $1,750.00$ \\
\hline 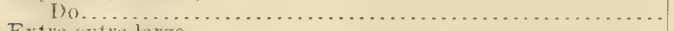 & 57 & 50 & 32.00 & $1,600,00$ \\
\hline Extra cestra large....... & $5 x$ & 60 & 51.00 & $3,060.00$ \\
\hline 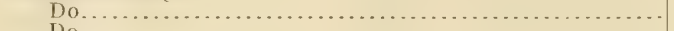 & 59 & 60 & 50.50 & $3,030.00$ \\
\hline 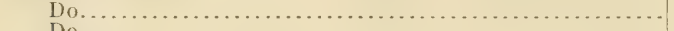 & 60 & 60 & 48.00 & 0.00 \\
\hline 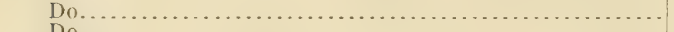 & 61 & 60 & 50.00 & 0.00 \\
\hline 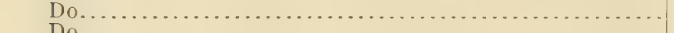 & 62 & 60 & 50.00 & 0.00 \\
\hline 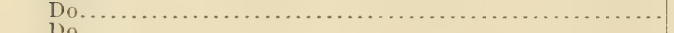 & 63 & 60 & 48.50 & 0.00 \\
\hline 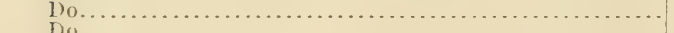 & 64 & 60 & 47.00 & .00 \\
\hline 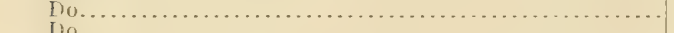 & 6.5 & 60 & 47.50 & $2,850.00$ \\
\hline 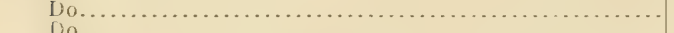 & 66 & 60 & 47.50 & $2,850,00$ \\
\hline 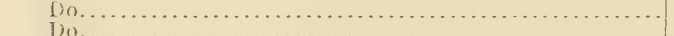 & 67 & 60 & 47.50 & $2,850.00$ \\
\hline & 65 & 60 & 51.50 & $3,090.00$ \\
\hline 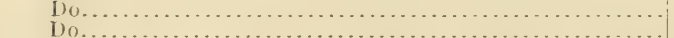 & 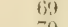 & 60 & 51.50 & $3,090.00$ \\
\hline & 70 & 38 & 54. & $2,052.00$ \\
\hline & 71 & 38 & 55.00 & $2,090.00$ \\
\hline 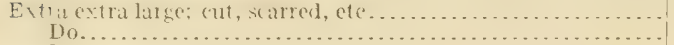 & $\because 2$ & 60 & 30.50 & $1,830.00$ \\
\hline & 73 & 60 & 30.50 & $1,830.00$ \\
\hline & 71 & 60 & 29.50 & $1,770.00$ \\
\hline 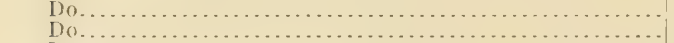 & 75 & 60 & 31.50 & 0.00 \\
\hline $\begin{array}{l}\mathrm{D}_{0}(1, \ldots \ldots \ldots \ldots \ldots \\
\mathrm{D}_{0} \ldots \ldots \ldots\end{array}$ & 76 & 60 & 29.50 & $1,770.00$ \\
\hline 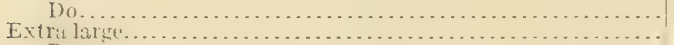 & 77 & 30 & 32.50 & 975.00 \\
\hline 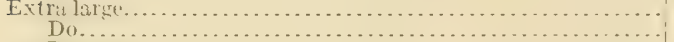 & $7 x$ & 70 & 49.00 & $3,430.00$ \\
\hline 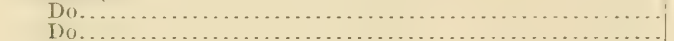 & 79 & 70 & 48.00 & $3,360.00$ \\
\hline 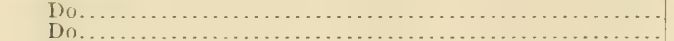 & 80 & 70 & 49.00 & $3,430.00$ \\
\hline & $\rightarrow 1$ & 70 & 51.50 & $3,605,00$ \\
\hline 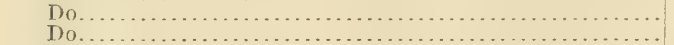 & $x^{2}$ & 70 & 50.00 & $3,500.00$ \\
\hline 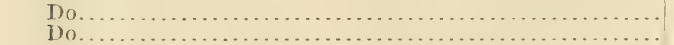 & $8 ; 3$ & 70 & 51.50 & $3,605,00$ \\
\hline $\begin{array}{l}\mathrm{D} \\
\mathrm{D} 0{ }_{0}\end{array}$ & sit & 70 & 50.50 & $3,535,00$ \\
\hline $\begin{array}{l}\mathrm{D} 0 \\
\mathrm{D} 0 \ldots \ldots \ldots \ldots\end{array}$ & $5 . j$ & 70 & 55.00 & $3,850.00$ \\
\hline 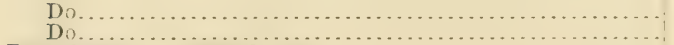 & $\times i$ & 70 & $5 \div .00$ & $3,780.00$ \\
\hline 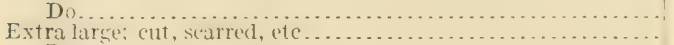 & $\$ 7$ & 53 & 53.50 & $2,835,00$ \\
\hline 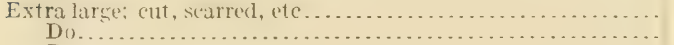 & Si & 70 & 26.00 & $1,820,00$ \\
\hline $\begin{array}{l}\mathrm{D}(\mathrm{D} \\
\mathrm{D} 0 \ldots \ldots \ldots \ldots \ldots \ldots \ldots \ldots \ldots \ldots \ldots\end{array}$ & 49 & 70 & 27.00 & $1,890,00$ \\
\hline (n....... & 90 & 70 & 29.00 & 30.00 \\
\hline 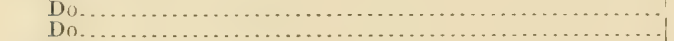 & 91 & 70 & 31.00 & $2,170.00$ \\
\hline n. & 92 & 70 & 31.00 & $2,170,00$ \\
\hline 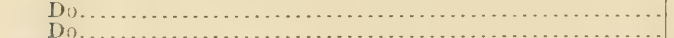 & 93 & 70 & 31.00 & $2,170.00$ \\
\hline 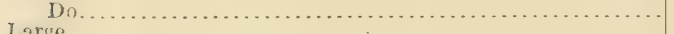 & 41 & 18 & 31.00 & $1, f \& 5,00$ \\
\hline 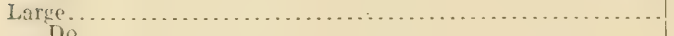 & 9.5 & (4) & Ax. (II) & $3, \times 10.110$ \\
\hline 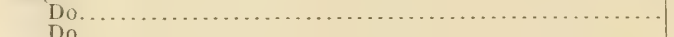 & 36 & 80 & 50.00 & $4,000,00$ \\
\hline 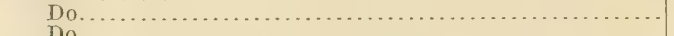 & 97 & S0 & 49.00 & $3,920.00$ \\
\hline 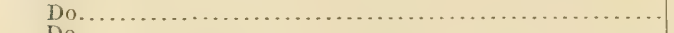 & $9 s$ & 80 & 50.00 & $4,000.00$ \\
\hline Do............... & 39 & 80 & 49.50 & $3,960.00$ \\
\hline$\ldots \ldots \ldots \ldots$ & $10)$ & 80 & 50.50 & $4,040.00$ \\
\hline Do............. & 11)I & 80 & 50.00 & $4,000.00$ \\
\hline$\ldots \ldots \ldots \ldots \ldots \ldots \ldots \ldots \ldots \ldots \ldots$ & $10: 2$ & 80 & $\$ 9.50$ & $3,960.00$ \\
\hline$\ldots \ldots \ldots \ldots \ldots \ldots \ldots \ldots \ldots \ldots$ & $10: 3$ & 80 & 50.00 & $4,000,00$ \\
\hline 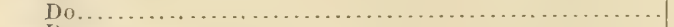 & $10 \pm$ & 50 & 50.00 & $2,500,00$ \\
\hline 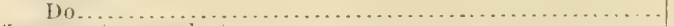 & 10 & 36 & 51.50 & $1,854.00$ \\
\hline 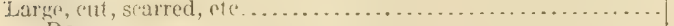 & $104 j$ & 80 & 27.50 & $2,200.00$ \\
\hline 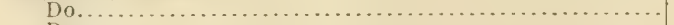 & 107 & 80 & 28.00 & $2,240.00$ \\
\hline 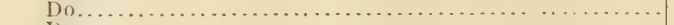 & $10 \mathrm{~s}$ & 80 & 30.00 & $2,400.00$ \\
\hline$\ldots \ldots \ldots \ldots \ldots \ldots$ & 109 & SO & 30.00 & $2,400,00$ \\
\hline 1$) 0 \ldots \ldots \ldots \ldots \ldots$ & 119 & S0 & 29.00 & $2,320,00$ \\
\hline $110 \ldots \ldots$ & 111 & 80 & 28.50 & $2,280.00$ \\
\hline D) $0 . \ldots \ldots \ldots \ldots$ & 112 & 80 & 29.50 & $2,360.00$ \\
\hline$\ldots \ldots \ldots \ldots \ldots$ & 113 & 80 & 30.09 & $2,400.00$ \\
\hline$\ldots \ldots \ldots \ldots, \cdots$, & 114 & $\mathrm{SO}$ & 29.50 & $2,-500,00$ \\
\hline 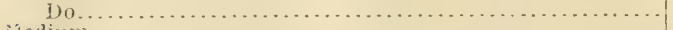 & 115 & 79 & 28,50 & $2,251,00$ \\
\hline 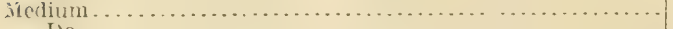 & 116 & 90 & 40.50 & $3,645.00$ \\
\hline 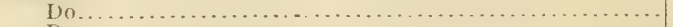 & 117 & 90 & 41.00 & 3.690 .010 \\
\hline 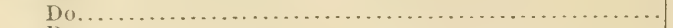 & $11 \mathrm{~s}$ & 90 & 40. 00 & $\therefore, 600,00$ \\
\hline 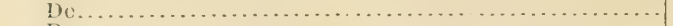 & 119 & 90 & 42.00 & $3,7 \times 0.00$ \\
\hline 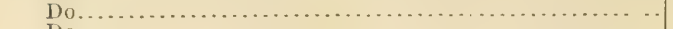 & 120 & 90 & 41.60 & $3,690.00$ \\
\hline 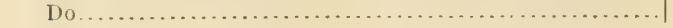 & 121 & 52 & 41.00 & $2,13: 00$ \\
\hline
\end{tabular}


Details of sale of 10,120 dressed, dycd, and machincd Pribilof Islunds fur-seal stins at St. Louis, Mo., on February 21, 1921-Continued.

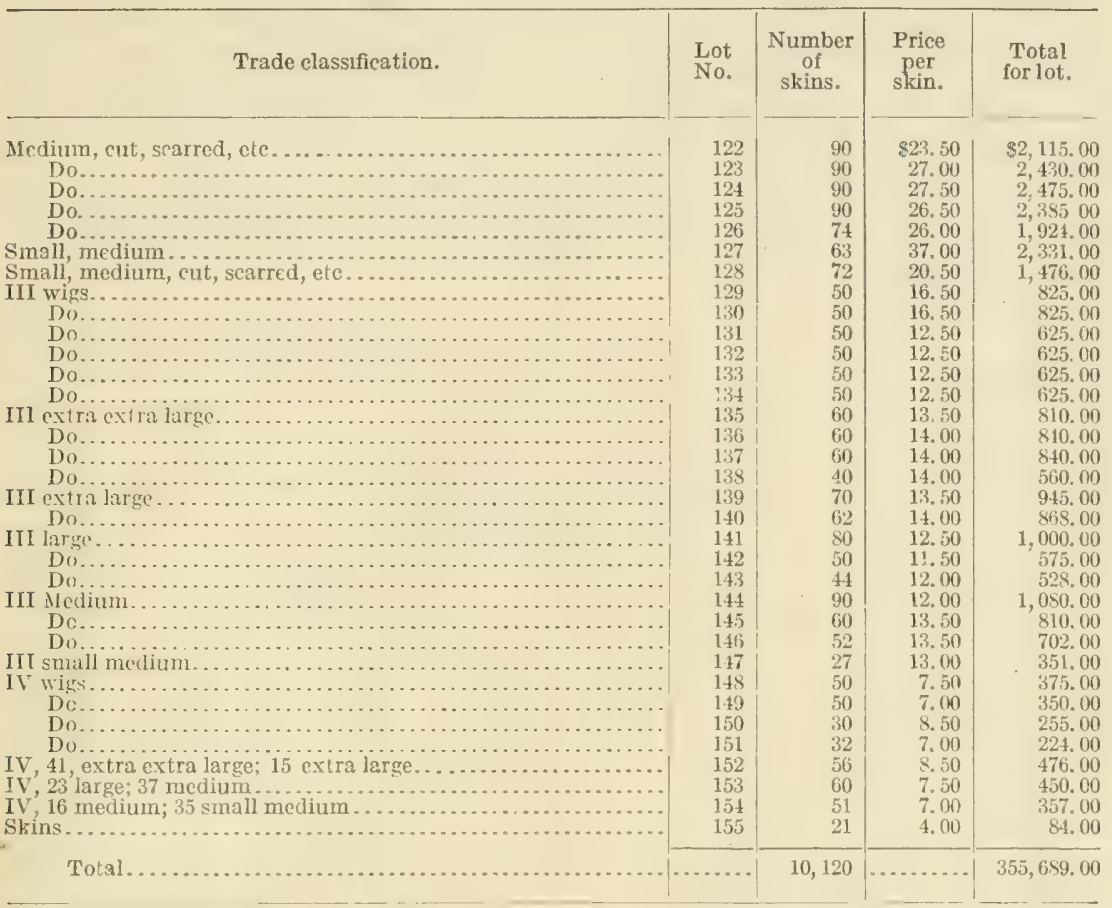

\section{XYI}

Hon. H. M. Sinth,

ST. Lours, March 15, 1921.

\section{Commissioner of Fisheries,}

Washington, D.C.

Dear Doctor Smith: Replying to your letter of February 4, addressed to Funsten Bros. \& ('o, in reference to the 19 skins which we are holding as exhibits of work done, also fur reference purposes in experiments, we suggest that we remit for these to the Government at \$35.14 per skin, the average price established at our recent sale, we to pay the dressing and dyeing charges.

We anticipated handling 100 skins of the 1919 catch for exhibition purposes, but so far have only put through 25 skins for this purpose. We suggest that we remit for these to the Government at $\$ 35.14$ per skin, we to pay the dressing and dyeing charges. It is more costly to us to prepure these skins in this manner, but we would be willing to charge only 15 per skin for the dressing and dyeing and dispose of them to retailers of sealskins at $\$ 50$ per skin. At this price we believe we could interest a number of retailers in different parts of the country, and we are confident that these exhibition skins will do much to create an interest in and a demand for Alaska fur seal skins which will redound to the benefit of the Government. As stated above, we have only finished 25 skins, but if they meet with the demand we expect them to we shall then want 75 more or as many as the trade can use for exhibition purposes.

We have contracted for page $I$ in the Fur Trade Review for a year, and the space shall be used to advertise Alaska sealskins and to urge a more general use of them. In addition to this we expect to do other circular advertising provided we have a large enough catch of sealskins each year. We hope to materially increase the demand for Alaska sealskins.

Awaiting your reply, we are very truly yours, 
March 19, 1921.

Gentlemen: In reply to your letter of the 15th instant, stating that you wish to purchase 19 fur-seal skins of the 1918 take and 25 of the 1919 take, which have been prepared as exhibition skins, at the rate of $\$ 35.14$, the average price established at the recent sale, you are advised that this price is acceptable to the department. 'This is with the understanding that the Government will not, as stated by you, be put to any expense for dressing and dyeing the skins. In order that transactions involving skins of the respectire years may be handled properly, please make separate remit. tances for the 1918 and 1919 takes of skins.

The department notes with interest that you plan to dispose of the 25 skins to retailers for publicity purposes. It is believed that your plans for advertising Alaska fur-seal skins will do much toward stimulating the demand, and that results will be most gratifying to all concerned.

Respectfully,

FOUKE FUR Co.,

E. F. SWEET, Assistant Secretary of Commerce.

St. Louis, Mo.

XVII.

Details of sale of 10,060 dressed, dyed, and machined Pribilof Islands fur-seal skins at St. Louis, Mo., on May 23, 1921.

\begin{tabular}{|c|c|c|c|c|}
\hline Trade classification. & $\begin{array}{l}\text { Lot } \\
\text { No. }\end{array}$ & $\begin{array}{c}\text { Number } \\
\text { of } \\
\text { skins. }\end{array}$ & $\begin{array}{l}\text { Price } \\
\text { per } \\
\text { skin. }\end{array}$ & $\begin{array}{l}\text { Total } \\
\text { for lot. }\end{array}$ \\
\hline 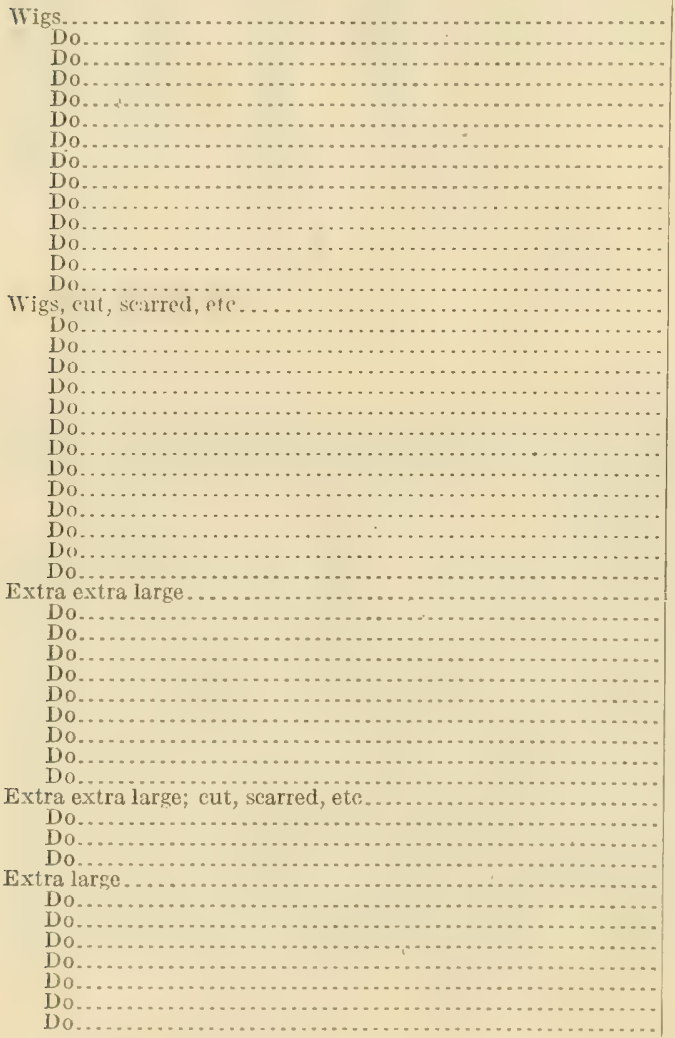 & $\begin{array}{r}1 \\
2 \\
3 \\
4 \\
5 \\
6 \\
7 \\
8 \\
9 \\
10 \\
11 \\
12 \\
13 \\
14 \\
15 \\
16 \\
17 \\
18 \\
19 \\
20 \\
21 \\
22 \\
23 \\
24 \\
25 \\
26 \\
27 \\
28 \\
29 \\
30 \\
31 \\
32 \\
33 \\
34 \\
35 \\
36 \\
37 \\
38 \\
39 \\
40 \\
41 \\
42 \\
43 \\
44 \\
45 \\
46 \\
47 \\
48 \\
49 \\
50\end{array}$ & $\begin{array}{l}50 \\
50 \\
50 \\
50 \\
50 \\
50 \\
50 \\
50 \\
50 \\
50 \\
50 \\
50 \\
50 \\
50 \\
50 \\
50 \\
50 \\
50 \\
50 \\
50 \\
50 \\
50 \\
50 \\
50 \\
50 \\
50 \\
50 \\
50 \\
60 \\
60 \\
60 \\
60 \\
60 \\
60 \\
60 \\
60 \\
60 \\
60 \\
60 \\
60 \\
60 \\
60 \\
70 \\
70 \\
70 \\
70 \\
70 \\
70 \\
70 \\
70 \\
\\
\\
\\
\\
\\
\\
\\
\\
\\
\end{array}$ & $\begin{array}{r}\$ 46.00 \\
42.00 \\
42.00 \\
41.00 \\
44.00 \\
45.00 \\
45.00 \\
43.00 \\
42.00 \\
45.00 \\
47.00 \\
46.00 \\
46.00 \\
47.00 \\
28.00 \\
29.00 \\
27.00 \\
26.00 \\
26.00 \\
26.00 \\
28.00 \\
28.00 \\
2.00 \\
28.00 \\
25.00 \\
28.00 \\
29.00 \\
30.00 \\
54.00 \\
56.00 \\
53.00 \\
55.00 \\
56.00 \\
56.00 \\
56.00 \\
58.00 \\
57.00 \\
56.00 \\
34.00 \\
35.00 \\
37.00 \\
37.00 \\
48.00 \\
48.00 \\
50.00 \\
50.00 \\
49.00 \\
49.00 \\
49.00 \\
50.00\end{array}$ & $\begin{array}{r}\$ 2,300.00 \\
2,100.00 \\
2,100.00 \\
2,050.00 \\
2,200.00 \\
2,250.00 \\
2,250.00 \\
2,150.00 \\
2,100.00 \\
2,250.00 \\
2,350.00 \\
2,300.00 \\
2,300.00 \\
2,350.00 \\
1,400.00 \\
1,450.00 \\
1,350.00 \\
1,300.00 \\
1,300.00 \\
1,300.00 \\
1,400.00 \\
1,400.00 \\
1,400.00 \\
1,400.00 \\
1,400.00 \\
1,400.00 \\
1,450.00 \\
1,500.00 \\
3,240.00 \\
3,360.00 \\
3,180.00 \\
3,300.00 \\
3,360.00 \\
3,360.00 \\
3,360.00 \\
3,480.00 \\
3,420.00 \\
3,360.00 \\
2,010.00 \\
2,100.00 \\
2,220.00 \\
2,220.00 \\
3,360.00 \\
3,360.00 \\
3,500.00 \\
3,500.00 \\
3,430.00 \\
3,430.00 \\
3,430.00 \\
3,500.00\end{array}$ \\
\hline
\end{tabular}


Details of sule of 10,060 dressed, dyed, and machinel Pribilof Islands fur-seal blins at St. Louis, Mo., on May 23, 1921-Continued.

Trade classification

Extra large.

$$
\text { Do }
$$

Extra large; cut, scarred, etc.

Large

Do.

Do

Do.

Do...

Do.

Do.

Do.

Do

Do.

Do.

Do.

Do

Do.

Do.

Do.

Do.

Do

Large: cut, scarred, et

Do.

Do.

Do.

Do.

Do

Do.

Do.

Do

Medium

Do.

Do.

Do.
Do.
Do.

Do.

Do

Dn.

Do.

Do. . .

Do.

Do.

Do.

Do.

Do.

$\mathrm{D}(\mathrm{c}$.

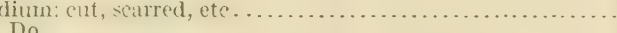

Do...

Do.

Do.

Dก.....

Do.

Do.......

Small miclitum.

Do.

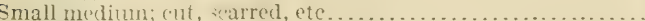

1 wig

Do

Do.

\begin{tabular}{l|c|c} 
Lot & $\begin{array}{c}\text { Number } \\
\text { of } \\
\text { skins. }\end{array}$ & $\begin{array}{c}\text { Price } \\
\text { per } \\
\text { skin. }\end{array}$
\end{tabular} \mid

Total

for lot.

\section{列}

\section{$\$ 3,570, C 0$}

$3,640.00$

$2,3 \pm 5.00$

$2,415.00$

$2,450.00$

2, 520.00

3, 280.00

$3,360.00$

$3,360.00$

$3,440.00$

$3,440.00$

$3,360.00$

$3,360.00$

$3,410.00$

$3,410.00$

$3,440.00$

$3,520.00$

$3,520.00$

520.00

$3,140.00$

$3,110.00$

3, 600.00

$3,640,00$

$3,520,00$

$3,520.00$

$3,520.00$

$3,560.00$

$3,520.00$

$3,600.00$

$3,520.00$

$2,720,00$

$2,720.00$

$2,680.00$

$2,720.00$

$2,680.00$

$2,720.00$

$2,720.00$

2, 920.00

2, 810.00

2, 850.00

$2,810.00$

$3,330.00$

3, 210.00

$3,060.00$

$3,060.00$

$3,105.00$

3,15000

$3,105.00$

$3,060.00$

$3,150.00$

$3,015,00$

3, 105.00

$3,150,00$

$3,195.00$

$2,970.00$

3, 015.00

$3,015.00$

$3,015.00$

$3,015.00$

2, 970.00

2, 970.00

$2,970.00$

$2,430.00$

$2,475.00$

$2,430.00$

$2,565.00$

$2,475.00$

$2,475.00$

$2,565.00$

$2,565.00$

2, 565.00

$2,430.00$

2, 520.00

$2,100.00$

$2,000.00$

625.00

800.00

675.00 
Details of sale of 10,060 dressed, dyed, and machined Pribilof Islands fur-seal skins at St. Louis, Mo., on May 23, 1921-Continued.

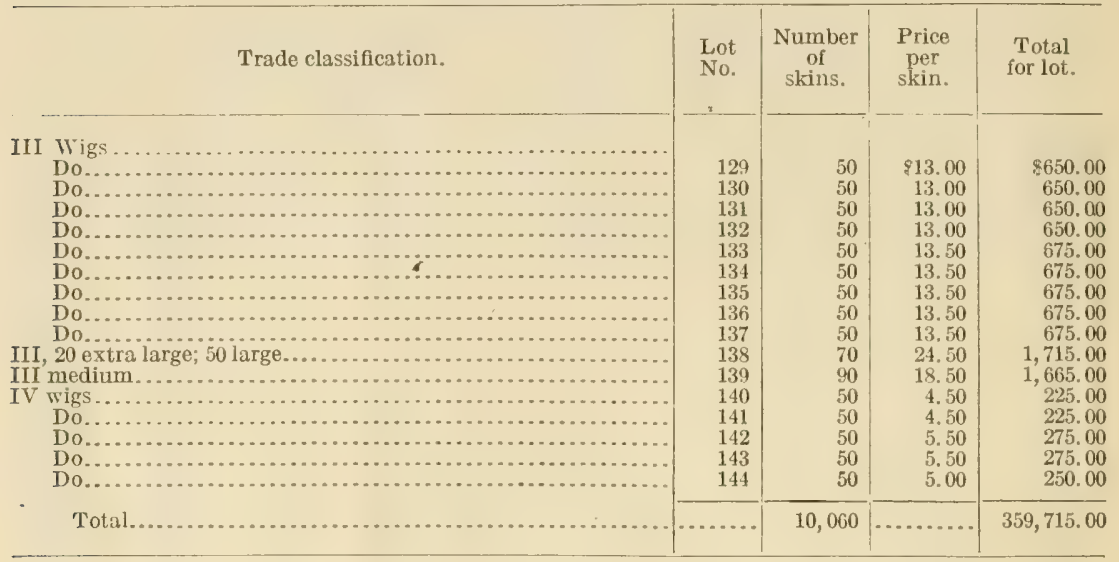

\section{XVIII.}

Details of sale of 10,778 dressed, dyed, and machined Pribilof Islands fur-seal skins at St. Louis, Mo., on September 28, 1921.

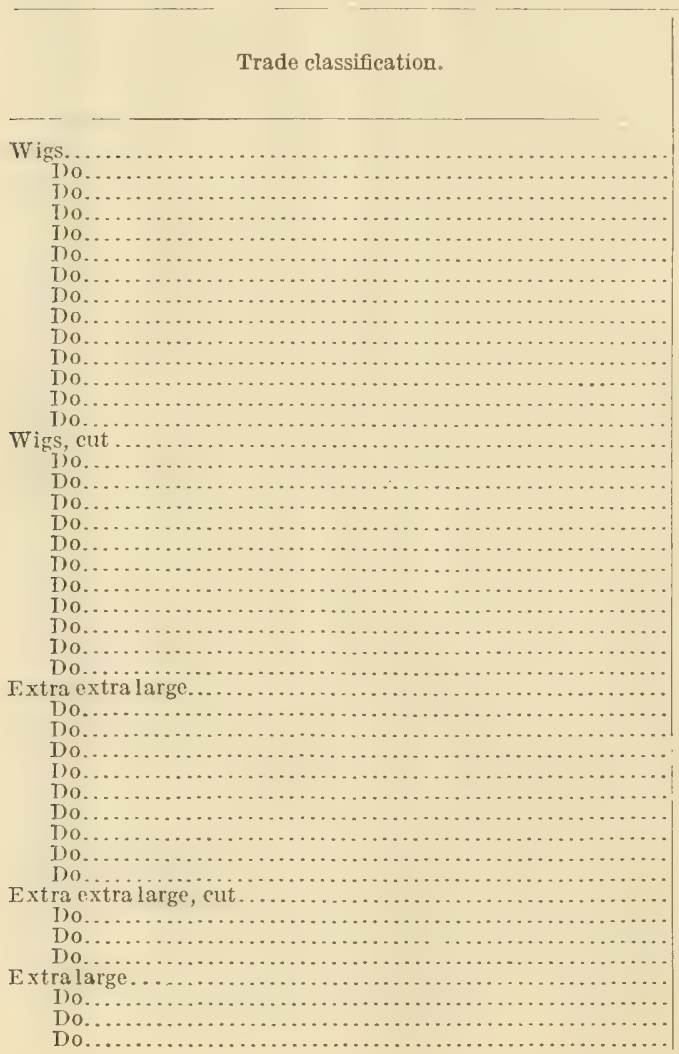

\begin{tabular}{|c|c|c|c|}
\hline $\begin{array}{l}\text { Lot } \\
\text { No. }\end{array}$ & $\begin{array}{c}\text { Number } \\
\text { of } \\
\text { skins. }\end{array}$ & $\begin{array}{c}\text { Price } \\
\text { per } \\
\text { skin. }\end{array}$ & $\begin{array}{l}\text { Total } \\
\text { for lot. }\end{array}$ \\
\hline 1 & 50 & $\$ 36.00$ & $\$ 1,800.00$ \\
\hline 2 & 50 & 35.00 & $1,750.00$ \\
\hline 3 & 50 & 39.00 & $1,950.00$ \\
\hline 4 & 50 & 36.00 & $1,800.00$ \\
\hline 5 & 50 & 38,00 & $1,900,00$ \\
\hline 6 & 50 & 36.00 & $1,800.00$ \\
\hline 7 & 50 & 39.00 & $1,950.00$ \\
\hline 8 & 50 & 37.00 & $1,850.00$ \\
\hline 9 & 50 & 35.00 & $1,750.00$ \\
\hline 10 & 50 & 38.00 & $1,900.00$ \\
\hline 11 & 50 & 39.00 & $1,950.00$ \\
\hline 12 & 50 & 41.00 & $2,050.00$ \\
\hline 13 & 50 & 38.00 & $1,930.00$ \\
\hline 14 & 50 & 39.00 & $1,950.00$ \\
\hline 15 & 50 & 23.00 & $1,150.00$ \\
\hline 16 & 50 & 23.00 & $1,150.00$ \\
\hline 17 & 50 & 23.00 & $1,150.00$ \\
\hline 18 & 50 & 25.00 & $1,250.00$ \\
\hline 19 & 50 & 25.00 & $1,250.00$ \\
\hline 20 & 50 & 24.50 & $1,225.00$ \\
\hline 21 & 50 & 25.50 & $1,275.00$ \\
\hline 22 & 50 & 26.00 & $1,300.00$ \\
\hline 23 & 50 & 27.50 & $1,375.00$ \\
\hline 24 & 50 & 25.00 & $1,250.00$ \\
\hline 25 & 50 & 27. 00 & $1,350,00$ \\
\hline 26 & 50 & 27.00 & $1,350.00$ \\
\hline 27 & 60 & 54.00 & $3,240,00$ \\
\hline 28 & 60 & 52.00 & $3,120,00$ \\
\hline 29 & 60 & 54.00 & $3,240.00$ \\
\hline 30 & 60 & 52,00 & $3,120.00$ \\
\hline 31 & 60 & 54.50 & $3,270,00$ \\
\hline 32 & 60 & 54.00 & $3,240.00$ \\
\hline 33 & 60 & 55.50 & $3,330,00$ \\
\hline 34 & 60 & 55.00 & $3,300,00$ \\
\hline 35 & 69 & 55.00 & $3,300.00$ \\
\hline 36 & 30 & 53.00 & - $\quad 1,590.00$ \\
\hline 37 & 60 & 33.00 & $1,980.00$ \\
\hline 38 & 60 & 34.50 & $2,070.00$ \\
\hline 39 & 60 & 36.00 & $2,160.00$ \\
\hline 40 & 60 & 36.50 & $2,190,00$ \\
\hline 41 & 70 & 42.50 & $2,975.00$ \\
\hline 42 & 70 & 43.00 & $3,010.00$ \\
\hline 13 & 70 & 44.50 & $3,115.00$ \\
\hline 44 & 70 & 43.50 & $3,045,00$ \\
\hline
\end{tabular}


Detwils of sule of 10,778 dressed. dyed. and machimed Pribilof Islands fur-seal slins at St. Louis, Mo., September 28, 1921-Continued.

\begin{tabular}{|c|c|c|c|c|}
\hline Trade classification. & $\begin{array}{l}\text { Lot } \\
\text { No. }\end{array}$ & $\begin{array}{c}\text { Number } \\
\text { of } \\
\text { skins. }\end{array}$ & $\begin{array}{c}\text { Price } \\
\text { per } \\
\text { shin. }\end{array}$ & $\begin{array}{l}\text { Total } \\
\text { for lot. }\end{array}$ \\
\hline Extra large......... & 45 & 70 & 845.50 & $83,185,00$ \\
\hline 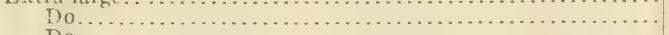 & 46 & 70 & 44.00 & $\begin{array}{r}\$ 3,185.00 \\
3,080.00\end{array}$ \\
\hline$\ldots \ldots \ldots \ldots \ldots \ldots \ldots \ldots \ldots \ldots \ldots \ldots \ldots \ldots$ & 47 & 70 & 48.00 & $3,360.00$ \\
\hline$\ldots \ldots \ldots \ldots \ldots \ldots \ldots \ldots \ldots \ldots \ldots \ldots \ldots \ldots$ & 48 & 70 & 46.00 & $3,220.00$ \\
\hline$\ldots \ldots \ldots \ldots \ldots \ldots \ldots \ldots \ldots \ldots \ldots \ldots \ldots \ldots \ldots$ & 49 & 70 & 47.00 & $3,290.00$ \\
\hline 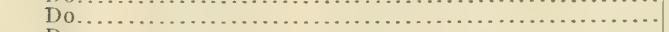 & 50 & 70 & 49.00 & $3,430 . \mathrm{CO}$ \\
\hline 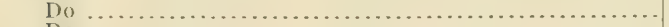 & 51 & 70 & 46.50 & $3,255.00$ \\
\hline 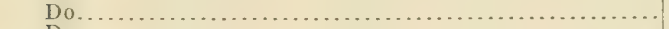 & 52 & 70 & 47.50 & $3,325.00$ \\
\hline 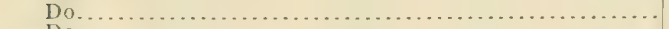 & 53 & 70 & 47.50 & $3,325.00$ \\
\hline$\ldots \ldots \ldots \ldots \ldots \ldots \ldots \ldots \ldots \ldots \ldots \ldots \ldots \ldots$ & 54 & 50 & 46.00 & $2,300.00$ \\
\hline 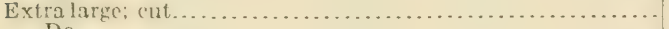 & 55 & 70 & 34.00 & $2,380.00$ \\
\hline 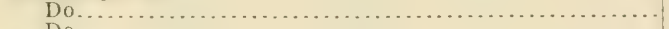 & 56 & 70 & 33.50 & $2,345.00$ \\
\hline 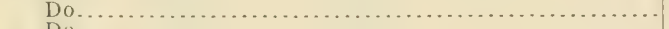 & 57 & 70 & 34.00 & $2,380.00$ \\
\hline 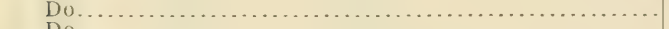 & 58 & 70 & 33.50 & $2,345.00$ \\
\hline 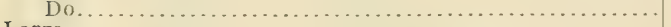 & 59 & 70 & 34.50 & $2,415.00$ \\
\hline 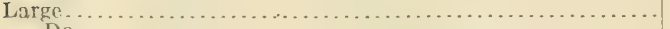 & 60 & 80 & 42.50 & $3,400.00$ \\
\hline 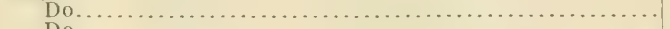 & 61 & 80 & 41.00 & $3,280.00$ \\
\hline 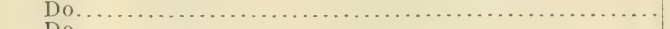 & 62 & 80 & 42.50 & $3,400.00$ \\
\hline 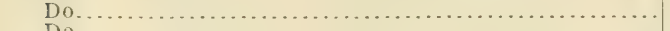 & 63 & 80 & 41.00 & .00 \\
\hline 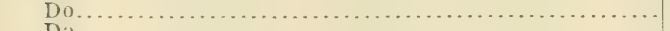 & 64 & 80 & 39.50 & 0.00 \\
\hline 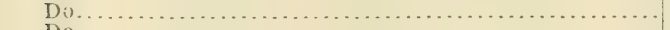 & 65 & 80 & 40.00 & $3,200.00$ \\
\hline 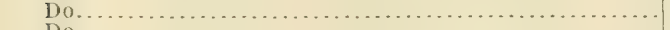 & 66 & 80 & 41.00 & 9.00 \\
\hline 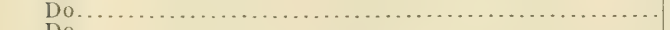 & 67 & 80 & 39.00 & .00 \\
\hline 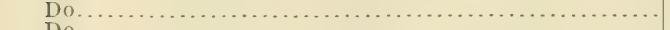 & 68 & 80 & 39.00 & .00 \\
\hline 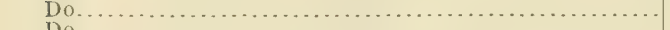 & 69 & 80 & 00 & .00 \\
\hline 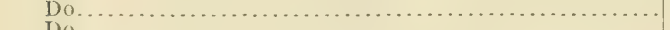 & 70 & 80 & 39.00 & $3,120.00$ \\
\hline 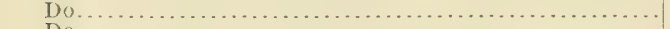 & 71 & 80 & 40.50 & $3,240,00$ \\
\hline 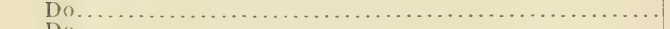 & 72 & 80 & 38.50 & $3,0 S 0.00$ \\
\hline 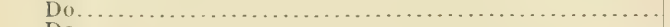 & 73 & 80 & 38.00 & $3,040.00$ \\
\hline 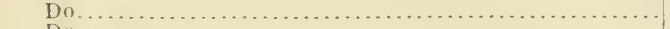 & 74 & 80 & 38.00 & $3,040.00$ \\
\hline 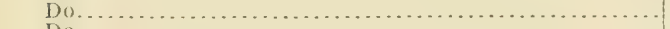 & 75 & 80 & 37.50 & $3,000.00$ \\
\hline 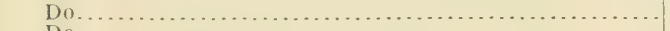 & 76 & 80 & 39.00 & $3,120.00$ \\
\hline 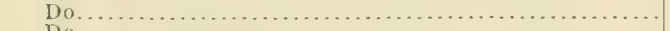 & 77 & 80 & 39.00 & $3,120.00$ \\
\hline 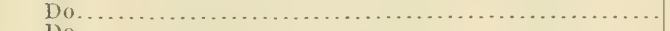 & 78 & 80 & 37.50 & $3,000.00$ \\
\hline 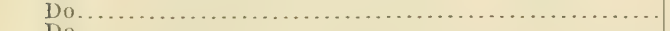 & 79 & 80 & 37.50 & $3,000.00$ \\
\hline 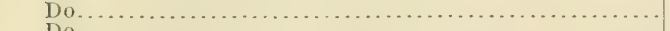 & so & 80 & 37.50 & $3,000.00$ \\
\hline 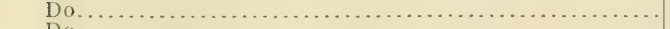 & 81 & 80 & 38.00 & $3,040.00$ \\
\hline 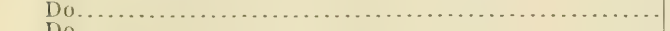 & 82 & 80 & 39 & $3,120.00$ \\
\hline 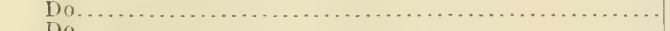 & 83 & 80 & 38 & $3,0.40 .00$ \\
\hline 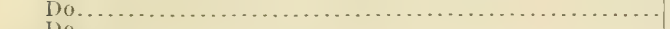 & 84 & 80 & 90 & 0.00 \\
\hline $100 \ldots \ldots \ldots \ldots \ldots$ & 85 & 80 & 0 & 0.00 \\
\hline$\ldots \ldots \ldots \ldots \ldots \ldots \ldots \ldots \ldots \ldots \ldots \ldots$ & 86 & 40 & 00 & .00 \\
\hline$\ldots \ldots \ldots \ldots \ldots \ldots \ldots \ldots \ldots \ldots$ & 57 & So & 00 & .00 \\
\hline 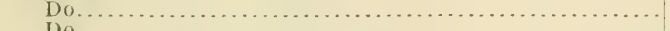 & 88 & 80 & 0 & .00 \\
\hline 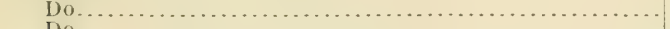 & $\$ 9$ & 80 & 30. & $2,400.00$ \\
\hline Do $0 \ldots \ldots \ldots \ldots$ & 90 & 80 & 10 & $2,400.00$ \\
\hline 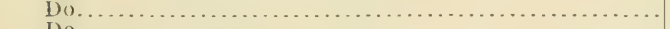 & 91 & 80 & 50 & $2,520.00$ \\
\hline 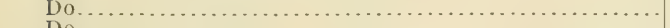 & 92 & 80 & 31. & $2,520.00$ \\
\hline 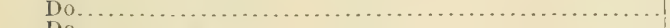 & 93 & 80 & 31.00 & $2,480.00$ \\
\hline 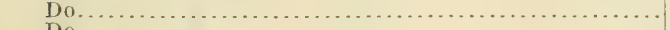 & 94 & so & 31.00 & $2,480.00$ \\
\hline Do................ & 95 & 80 & 31.50 & $2,520.00$ \\
\hline 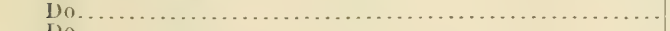 & 36 & 80 & 31.50 & $2,320.00$ \\
\hline 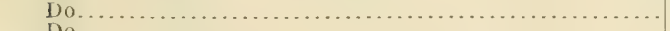 & 97 & 80 & 31.00 & $2,450.00$ \\
\hline$D o \ldots \ldots \ldots \ldots \ldots$ & 98 & 80 & 31.50 & $2,520.00$ \\
\hline Do $\ldots \ldots$ & 99 & 60 & 30.50 & $1,8.30 .00$ \\
\hline díediums. . . . . . . . . & 100 & 90 & 31.00 & 0.00 \\
\hline 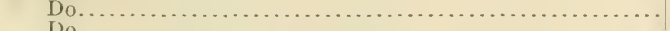 & 101 & 90 & 30. & 0.00 \\
\hline 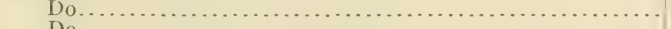 & 102 & 90) & 30.50 & $2,745.00$ \\
\hline 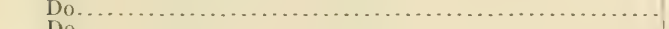 & 103 & 90 & 30 & .00 \\
\hline Do................ & 104 & 90 & 30 & 00 \\
\hline 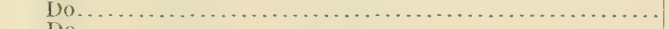 & 105 & 90 & 30 & $2,700.00$ \\
\hline 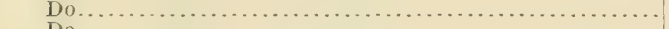 & 106 & 90 & & $2,700.00$ \\
\hline 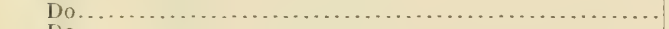 & 107 & 90 & 29 & $2,655.00$ \\
\hline 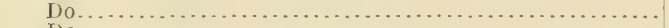 & 108 & 90 & 29. & 2,6 \\
\hline 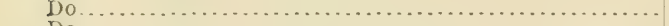 & 109 & 90 & 29. & $2,610.00$ \\
\hline 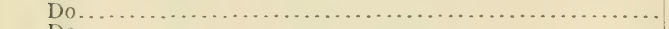 & 110 & 90 & 28 & $2,520.00$ \\
\hline 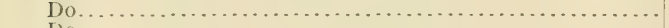 & 111 & 90 & 29.50 & $2,655.00$ \\
\hline 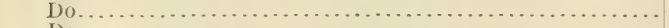 & 112 & 90 & 30 . & $2,700.00$ \\
\hline 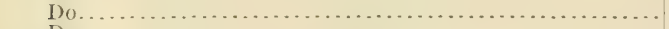 & 113 & 90 & 30 & $2,700.00$ \\
\hline$\ldots \ldots \ldots \ldots \ldots . .$. & 114 & 90 & & 5.00 \\
\hline Do & 115 & 90 & 30 & 00 \\
\hline Medium; cut.. & 116 & 90 & 22. & 0.00 \\
\hline 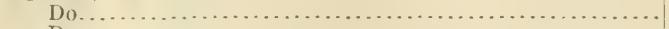 & 117 & 90 & 22.00 & 0.00 \\
\hline Do................... & 118 & 90 & 22.00 & 0.00 \\
\hline 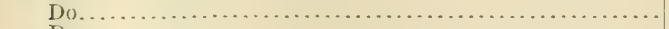 & 119 & 90 & 22.50 & 5.00 \\
\hline 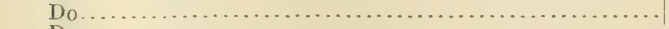 & 120 & 90 & 22.00 & 0.00 \\
\hline 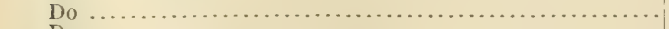 & 121 & 90 & 22.00 & 30. 00 \\
\hline 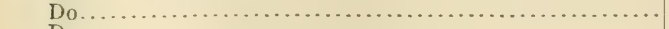 & 122 & 90 & 22.00 & 0.00 \\
\hline 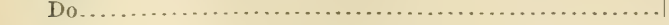 & 123 & 90 & 22.00 & $1,980.00$ \\
\hline
\end{tabular}


Details of sale of 12,777 dressed, dyed, and machined Pribilof Islands fur-seal skins at St. Louis, Mo., on A pril 3, 1922-Continued.

\begin{tabular}{|c|c|c|c|c|}
\hline Trade classification. & $\begin{array}{l}\text { Lot } \\
\text { No. }\end{array}$ & $\begin{array}{c}\text { Number } \\
\text { of } \\
\text { skins. }\end{array}$ & $\begin{array}{c}\text { Price } \\
\text { per } \\
\text { skill. }\end{array}$ & $\begin{array}{l}\text { Total } \\
\text { for lot. }\end{array}$ \\
\hline Lar & 32 & 80 & $\$ 39.00$ & $\$ 3,120,00$ \\
\hline 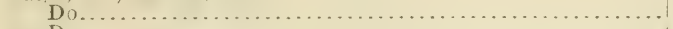 & 33 & 80 & 41.00 & $3,280.00$ \\
\hline 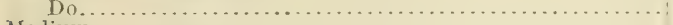 & 34 & 80 & 41.50 & $3,320.00$ \\
\hline 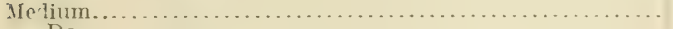 & 35 & 90 & 41.00 & $3,690,00$ \\
\hline 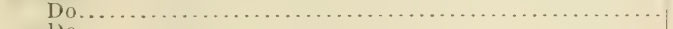 & 36 & 90 & 39.50 & $3,555.00$ \\
\hline 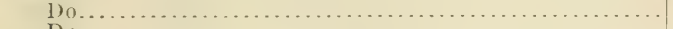 & 37 & 90 & 37.50 & $3,375,00$ \\
\hline 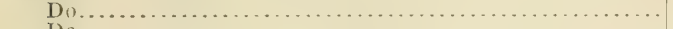 & 38 & 90 & 37.50 & 5.00 \\
\hline 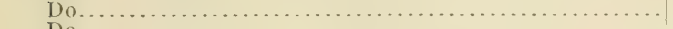 & 39 & 90 & 36.00 & .00 \\
\hline 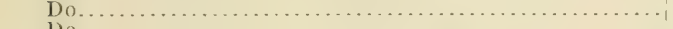 & 40 & 90 & 36.50 & 5.00 \\
\hline 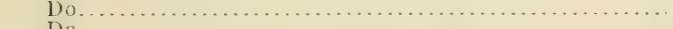 & 11 & 90 & 36.50 & .00 \\
\hline 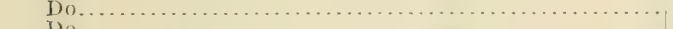 & 42 & 90 & 37.50 & .00 \\
\hline 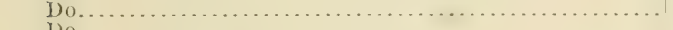 & 43 & 90 & 50 & 00 \\
\hline 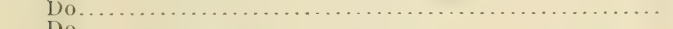 & 41 & 90 & 37.00 & 0.00 \\
\hline 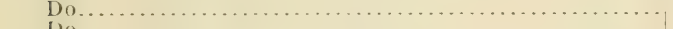 & 45 & 90 & 00 & 0.00 \\
\hline 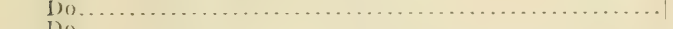 & 46 & 90 & 37.00 & $3,330.00$ \\
\hline 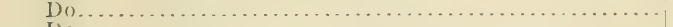 & 47 & 90 & 36. 00 & $3,240.00$ \\
\hline 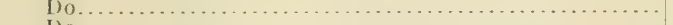 & 48 & 90 & 36.50 & $3,285.00$ \\
\hline I) & 49 & 90 & 36.50 & $3,285.00$ \\
\hline 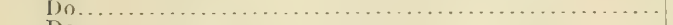 & 50 & 90 & 38.00 & $3,420.00$ \\
\hline 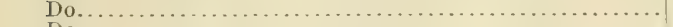 & 51 & 90 & 37.00 & $3,330,00$ \\
\hline 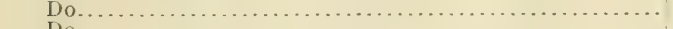 & 52 & 90 & 38.00 & $3,420.00$ \\
\hline 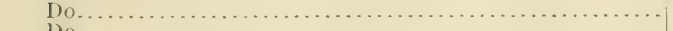 & 53 & 90 & 37.00 & $3,330.00$ \\
\hline 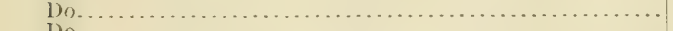 & $5 \pm$ & 90 & 37.00 & $3,330.00$ \\
\hline 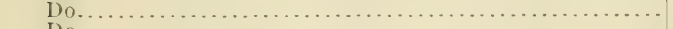 & 55 & 90 & 36.00 & $3,240.00$ \\
\hline 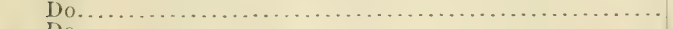 & 56 & 90 & 38.50 & .00 \\
\hline 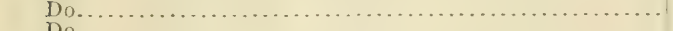 & 57 & 90 & 39.00 & .00 \\
\hline$\ldots \ldots \ldots \ldots \ldots \ldots \ldots$ & 58 & 90 & 37.50 & .00 \\
\hline$\ldots \ldots \ldots \ldots \ldots \ldots \ldots \ldots \ldots, \ldots$, & 59 & 90 & 37.50 & 00 \\
\hline 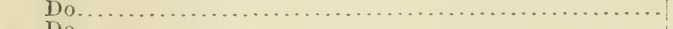 & 60 & 90 & 37. & .00 \\
\hline 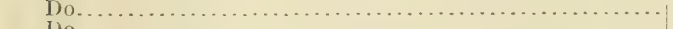 & 61 & 90 & 37.00 & .00 \\
\hline$\ldots \ldots \ldots \ldots \ldots \ldots \ldots \ldots \ldots \ldots \ldots$ & 62 & 90 & 37.50 & 00 \\
\hline$\ldots \ldots \ldots \ldots \ldots \ldots \ldots \ldots \ldots$, & 63 & 90 & 37. & 00 \\
\hline$\ldots \ldots \ldots \ldots \ldots \ldots \ldots \ldots \ldots \ldots \ldots$ & 64 & 90 & 90 & 0.00 \\
\hline$\ldots \ldots \ldots \ldots \ldots \ldots \ldots \ldots \ldots \ldots \ldots$ & 65 & 90 & 37 & 3, \\
\hline$\cdots \ldots \ldots \ldots \ldots \ldots \ldots \ldots \ldots \ldots \ldots \ldots$ & 66 & 90 & 37. & 0.00 \\
\hline 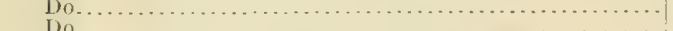 & 67 & 90 & 36. & 10.00 \\
\hline 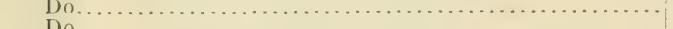 & 68 & 90 & 37. & $3,375.00$ \\
\hline 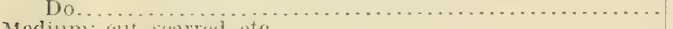 & 69 & 90 & 37.00 & $3,330.00$ \\
\hline 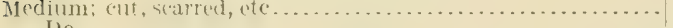 & 70 & 90 & 30.00 & $2,700.00$ \\
\hline 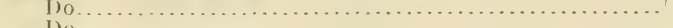 & 71 & 90 & 26.00 & $2,340.00$ \\
\hline 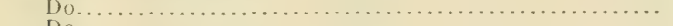 & 72 & 90 & 26.00 & $2,310.00$ \\
\hline 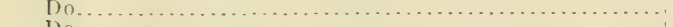 & 73 & 90 & 26.50 & $2,385.00$ \\
\hline 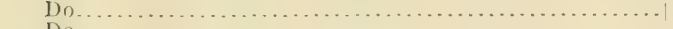 & $7 \pm$ & 90 & 26.00 & $2,340.00$ \\
\hline 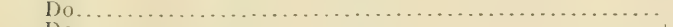 & 75 & 90 & 26.00 & $2,340.00$ \\
\hline 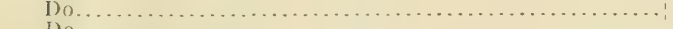 & 76 & 90 & 26. & $2,310,00$ \\
\hline 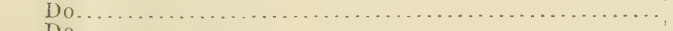 & 77 & 90 & 26. 00 & $2,340.00$ \\
\hline ............. & 78 & 90 & 26.50 & 5.00 \\
\hline 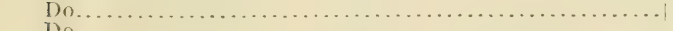 & 79 & 90 & 26.00 & $2,340.00$ \\
\hline 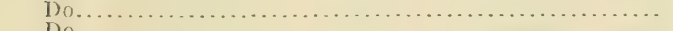 & 80 & 90 & 26.50 & $2,385.00$ \\
\hline 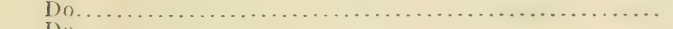 & 81 & 90 & 26. & $2,385.00$ \\
\hline 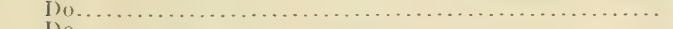 & $\$ 2$ & 90 & 26. & 5.00 \\
\hline 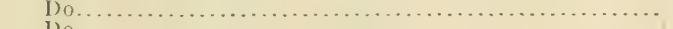 & 83 & 90 & 26. & 0.00 \\
\hline 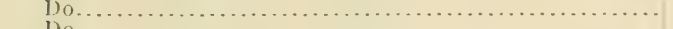 & 84 & 90 & 26.50 & 00 \\
\hline 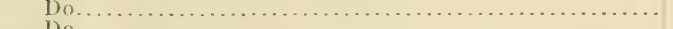 & 85 & 90 & 26. & 00 \\
\hline 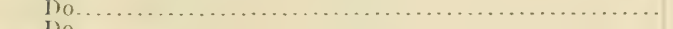 & 86 & 90 & 26. & 00 \\
\hline 1) $0 \ldots \ldots . .$. & 87 & 90 & 0 & 00 \\
\hline - mall me & 88 & 90 & 0 & 00 \\
\hline ........ & $\$ 9$ & 90 & 27. & 75.00 \\
\hline 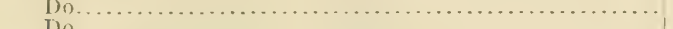 & 90 & 90 & 28 & 00 \\
\hline 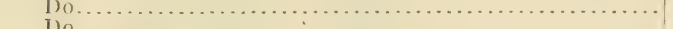 & 91 & 90 & 0 & 0 \\
\hline 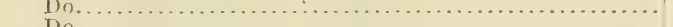 & 92 & 90 & 28 & 0 \\
\hline 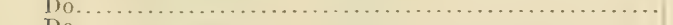 & 93 & 90 & 28 & 2,565 \\
\hline 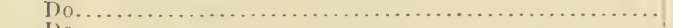 & $9 \frac{4}{2}$ & 90 & 28. & $2,565.00$ \\
\hline 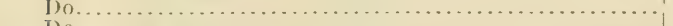 & 95 & 90 & 29. & 10.00 \\
\hline$\ldots \ldots \ldots \ldots \ldots \ldots \ldots$ & 96 & 70 & 28 & 00 \\
\hline $11 \mathrm{ml},(211 t, \mathrm{sin}$ & 97 & 90 & 23 & $2,115,00$ \\
\hline$\ldots \ldots \ldots \ldots \ldots \ldots$ & 98 & 90 & 22.50 & $2,025.00$ \\
\hline$\ldots \ldots \ldots \ldots$ & 99 & 90 & 22. & $2,025,00$ \\
\hline 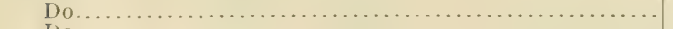 & 100 & 90 & 22. & 25.00 \\
\hline I) & 101 & 90 & 22 & 0.00 \\
\hline 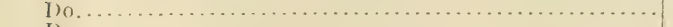 & 102 & 90 & 22. & 25.00 \\
\hline$\ldots \ldots \ldots \ldots \ldots \ldots$ & 103 & 90 & 23. & 70.00 \\
\hline 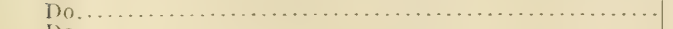 & 104 & 90 & 22. & 25.00 \\
\hline${ }^{\prime} \ldots \ldots \ldots \ldots \ldots \ldots \ldots \ldots \ldots \ldots \ldots \ldots$, & 105 & 90 & 22. & 25.00 \\
\hline 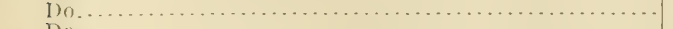 & 106 & 90 & 22. & $1.9 \times 0.00$ \\
\hline 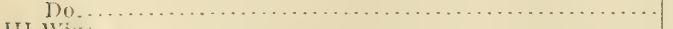 & 107 & 90 & 22. & $1,980.00$ \\
\hline$\ldots \ldots \ldots \ldots \ldots \ldots \ldots \ldots \ldots$ & 108 & 50 & 10. & 525.00 \\
\hline 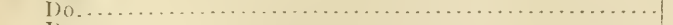 & 109 & 50 & & 500.00 \\
\hline & 110 & 30 & 10.00 & 300.00 \\
\hline
\end{tabular}


Details of sale of 12,777 dressed, dyed, and machined Pribilof Islands fur-seal skins at St. Louis, Mo., A pril 2, 1922-Continued.

\begin{tabular}{|c|c|c|c|c|}
\hline Trade classification. & $\begin{array}{l}\text { Lot } \\
\text { No. }\end{array}$ & $\begin{array}{c}\text { Number } \\
\text { of } \\
\text { skins. }\end{array}$ & $\begin{array}{l}\text { Price } \\
\text { per } \\
\text { skin. }\end{array}$ & $\begin{array}{l}\text { Total } \\
\text { for lot. }\end{array}$ \\
\hline III, 31 extra large: 19 lar & 111 & 50 & $\$ 15.50$ & $\$ 775.00$ \\
\hline um 43 small $\mathrm{mc}$ & 112 & 80 & 11.00 & 880.00 \\
\hline IV, wigs. . & 113 & 20 & 5.00 & 100.00 \\
\hline Wigs..... & 114 & 50 & 60.00 & $3,000.00$ \\
\hline Do. & 115 & 50 & 58.00 & $2,900.00$ \\
\hline Do.. & 116 & 50 & 58.00 & $2,900.00$ \\
\hline $\begin{array}{l}\text { Do } \\
\text { Wig: }\end{array}$ & 117 & 58 & 53.00 & $3,074.00$ \\
\hline $\begin{array}{l}\text { Wigs: cut, srarred, cte } \\
\text { Do................. }\end{array}$ & 118 & 50 & 29.50 & $1,475.00$ \\
\hline Do.......... & 119 & 50 & 28.50 & $1,425.00$ \\
\hline & 120 & 50 & 28.50 & $1,425.00$ \\
\hline $\begin{array}{l}\text { Do.. } \\
\text { Do. }\end{array}$ & 121 & 50 & 29.00 & $1,450.00$ \\
\hline $\begin{array}{l}\text { Do.. } \\
\text { Do... }\end{array}$ & 122 & 50 & 28.00 & $1,400.00$ \\
\hline $\begin{array}{l}\text { Do.. } \\
\text { Do.. }\end{array}$ & 123 & 50 & 28. & $1,400.00$ \\
\hline & 124 & 50 & 28.50 & $1,425.00$ \\
\hline $\begin{array}{l}\text { Do. } \\
\text { Do. }\end{array}$ & 125 . & 50 & 29.00 & $1,450.00$ \\
\hline $\begin{array}{l}\mathrm{Do} \\
\mathrm{DO}\end{array}$ & 126 & 50 & 30.00 & $1,500.00$ \\
\hline $\begin{array}{l}\text { Do } \\
\text { Do }\end{array}$ & 127 & 50 & 29.00 & $1,450.00$ \\
\hline $\begin{array}{l}\text { J) } 0 . \\
\text { Do. }\end{array}$ & 128 & 50 & 29.50 & $1,475.00$ \\
\hline $\begin{array}{l}\text { Do...... } \\
\text { Extra extra }\end{array}$ & 129 & 22 & 31.50 & 693.00 \\
\hline Extra extra large.......... & 130 & 33 & 62.00 & $2,045.00$ \\
\hline large; cut, scar & 131 & 50 & 44.00 & $2,200.00$ \\
\hline Do & 132 & 27 & 43.50 & $1,174.50$ \\
\hline e........ & 133 & 44 & 57.00 & $2,508.00$ \\
\hline $\begin{array}{l}\text { Extra large; cut, sc } \\
\text { Do }\end{array}$ & 134 & 50 & 41.00 & $2,050.00$ \\
\hline 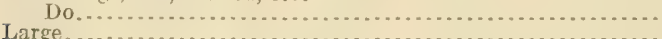 & 135 & 23 & 40.00 & 920.00 \\
\hline Large ${ }_{\text {Do....................... }}$ & 13 & 50 & 48. 00 & $2,400.00$ \\
\hline & 137 & 25 & 53.50 & $1,337.50$ \\
\hline & 138 & 50 & 35.00 & $1,750.00$ \\
\hline 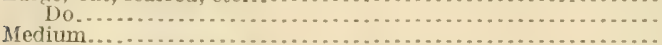 & 139 & 3 & 27.50 & $1,387.50$ \\
\hline eut scarred, et & 140 & 8 & 35.00 & $2,800.00$ \\
\hline $\begin{array}{l}\text { eut, scarred, ete } \\
\mathrm{m} ; 15 \text { small medi }\end{array}$ & 14 & 8 & 26 & $2,120.00$ \\
\hline $\begin{array}{l}22 \text { medium; } 15 \text { small medium } \\
16 \text { medium; cut, scarred, etc. } 12 \text { small }\end{array}$ & 142 & 37 & 26.50 & 980.50 \\
\hline & 143 & 28 & 21.00 & 588.00 \\
\hline III, wigs ${ }_{\text {Do }}$ & 144 & 50 & 6.50 & 325.00 \\
\hline (n) & 145 & 5 & 6.00 & 300.00 \\
\hline $\begin{array}{l}\text { Do } \\
\text { Do }\end{array}$ & 14 & 5 & 5. 50 & 275.00 \\
\hline $\begin{array}{l}\text { Do } \\
\text { Do }\end{array}$ & 147 & 5 & 5. 50 & 275.00 \\
\hline $\begin{array}{l}\text { Do } \\
\text { Do }\end{array}$ & 148 & 5 & 5.50 & 275.00 \\
\hline $\begin{array}{l}\text { Do } \\
\text { Do }\end{array}$ & 14 & & 5.50 & 275.00 \\
\hline I & 150 & 5 & 5.50 & 275.00 \\
\hline .......... & 151 & 5 & 6.00 & 300.00 \\
\hline (n........ & 15 & 5 & 6.00 & 300.00 \\
\hline$\cdots$ & 153 & 5 & 6.50 & 325.00 \\
\hline ... & 15 & 50 & 6.00 & 300.00 \\
\hline (n) & 15 & 50 & 6.50 & 325.00 \\
\hline ......... & 156 & 50 & 6.50 & 325.00 \\
\hline ....... & 15 & 5 & 6.50 & 325.00 \\
\hline Do... & $\begin{array}{l}158 \\
159\end{array}$ & $\begin{array}{l}50 \\
42\end{array}$ & $\begin{array}{l}7.00 \\
6.50\end{array}$ & $\begin{array}{l}350.00 \\
273.00\end{array}$ \\
\hline $\begin{array}{l}\text { III, } 14 \text { extra extra large; } 4 \text { extra large; } 7 \text { large; } 5 \text { mediums; } 25 \\
\text { small mediums. }\end{array}$ & 160 & 55 & 13.50 & 742.50 \\
\hline 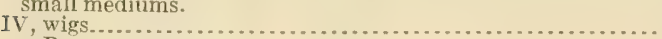 & 16 & 5 & 1.50 & 75.00 \\
\hline D & 162 & 5 & 2.00 & 100.00 \\
\hline ... & 16 & 5 & 2. & 100.00 \\
\hline & 10 & 5 & 1.50 & 75.00 \\
\hline I & 16 & 5 & 2.25 & 112.50 \\
\hline D & 16 & 5 & 3. & $150 .($ \\
\hline Dc & 16 & 5 & 2.25 & 112. \\
\hline & 16 & 5 & 2.25 & 112.50 \\
\hline & 16 & 5 & 1.50 & 75.0 \\
\hline D & 17 & 5 & 1.50 & 75. \\
\hline $\mathrm{D}$ & 17 & 5 & 1.75 & 87.5 \\
\hline & 17 & 5 & 1.50 & 75.00 \\
\hline Do. & 17 & 5 & 1.75 & 87. \\
\hline arge; 2 lar & 17 & 4 & 1.50 & 60.00 \\
\hline 1... & 17 & 27 & 8.25 & 222.75 \\
\hline 1 rejects, dressed and dyed. & 17 & 7 & .50 & 36.00 \\
\hline & 17 & 17 & .50 & 88. \\
\hline $\mathrm{Cu}$ & 178 & 11 & .50 & 58.5 \\
\hline Culls and rejects, washed and dried............................... & 179 & 121 & .25 & 30.25 \\
\hline \multirow{2}{*}{ 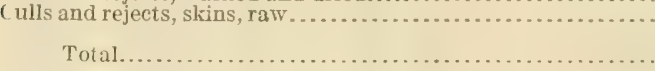 } & 180 & 92 & & 5.00 \\
\hline & & & & 388,288 \\
\hline
\end{tabular}


XX.

Statement of account as of January 1, 1922, of the United States with Great Britain and Japan, under the provisions of the North Pacific sealing convention of July \%, 1911, and the act of August 24, 1912.

\begin{tabular}{|c|c|c|c|}
\hline Date. & Item. & Credits. & Debits. \\
\hline $\begin{array}{l}\text { Sept. } 23,1912 \\
\text { Feb. } 14,1913\end{array}$ & $\begin{array}{l}\text { Cash ad vanced } \\
\text { Value of } 1,000 \text { sealskins taken in } 1912 \text { and sold in London, } \\
\text { Jant } 17,1913\end{array}$ & $\$ 31,672,13$ & $\$ 200,000.00$ \\
\hline Dec. 19,1913 & 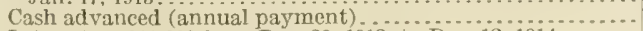 & & $10,000.00$ \\
\hline Dec, 12,1914 & Interest on $\$ 10,000$ from Dec. 20,1913 , to Dec. 12,1914 & & 392.36 \\
\hline Dec. 16,1915 & Interest on $\$ 20,000$ from Dec. 13,1914 , to Dec. 16 , & & $\begin{array}{r}0,000.00 \\
808.76\end{array}$ \\
\hline Do.......... & Cash advanced (annual payment) .................. & & $10,000.00$ \\
\hline Dec. 16,1916 & Interest on $\$ 30,000$ from Dec. 17,1915 , to Dec. 16,191 & & $1,200.00$ \\
\hline Jan. ${ }^{2}, 1918$ & $\begin{array}{l}\text { Cash advanced (annual payment) } \\
\text { Interest on } \$ 40,000 \text { from Dec. } 17,1916 \text {, to Jan. } 2,1918\end{array}$ & & $\begin{array}{r}10,000.00 \\
1,674.52\end{array}$ \\
\hline Do......... & 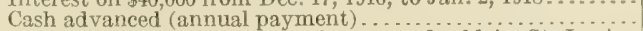 & & $10,000.00$ \\
\hline Jan. $\quad 9,1919$ & $\begin{array}{l}\text { Valie of } 1,000 \text { sealskins taken in } 1917 \text { and sold in St. Louis, } \\
\text { Mo., Apr. } 24,1919 \ldots \ldots \ldots \ldots\end{array}$ & $55,900.00$ & \\
\hline June 9,1919 & 15 per cent of net value of 4,277 seal skins taken in 1918 and & 2.64 & \\
\hline Oct. 27,1919 & $\begin{array}{l}15 \text { per cent of net value of } 9,055 \text { sealskins taken in } 1918 \text { and } \\
\text { sold in st. Lonis, } \$ 1 \text {., Sept. } 10,1919 . \ldots \ldots \ldots \ldots \ldots \ldots \ldots\end{array}$ & $101, \$ 47.77$ & \\
\hline Mar. 17,1920 & $\begin{array}{l}\text { Interest on } \$ 50,000 \text { from Jan. } 3,1918 \text {, to Oct. } 26,1919 \ldots \ldots . \\
15 \text { per cent of net value of } 9.100 \text { sealskins taken in } 1918 \text { and }\end{array}$ & & $3,621.92$ \\
\hline Do......... & $\begin{array}{l}\text { Interest on } \$ 29,420.50 \text { due the United States on unliquidated } \\
\text { balance from Oct. } 27,1919 \text {, to Mar. } 17,1920 \ldots . . . . . . . . .\end{array}$ & $165,822.06$ & \\
\hline Do & Cash paid by United States to settle & & 135,94 \\
\hline Nov. 10,1920 & 15 per cer & $391,104.60$ & 394 , \\
\hline June 7,1921 & $\begin{array}{l}\text { in } 1918 \text { and sold in St. Louis May } 10,19 \\
15 \text { per cent of net value of } 10,120 \text { sealskin }\end{array}$ & $49,800.83$ & \\
\hline Do & 1919 and sold in St. Louis Feb. 21, 1921. & $31,825.35$ & \\
\hline \multirow{4}{*}{ Oct. 15,1921} & $\begin{array}{l}15 \text { per } c \\
\text { and } \mathrm{s}\end{array}$ & 231.92 & \\
\hline & $\begin{array}{l}15 \text { per cent of net value of } 10,060 \text { sealskins taken in } 1919 \text { and } \\
\text { sold in St. Louis Mfay } 23,1921 \ldots \ldots \ldots \ldots \ldots \ldots \ldots \ldots \ldots \ldots \ldots\end{array}$ & $29,471.94$ & \\
\hline & $\begin{array}{l}\text { Amount paid Great Britain, as } \\
\text { Amount paid Japan, as above. }\end{array}$ & $\begin{array}{l}50.5,434.64 \\
50.5,431.64\end{array}$ & \\
\hline & $A m$ & 9.28 & \\
\hline
\end{tabular}

\section{XXI.}

Memorandum statement regarding the sale of $3,7 \% 3$ sealskins taken on the Pribilof Istands, Alaska, in calendar year 1912 and sold in London on January 17, 1913.

Gross amount of sale.

$\$ 141,289.58$

Expenses of sale, transportation, etc.:
Cash discount
$\$ 3,532.23$
Commission.
$5,510.28$
Marine insurance................................

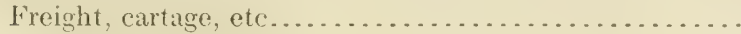
$1,064.95$
364.17

Net receipts

$10,471.63$

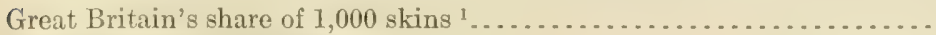

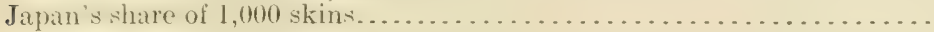

$34,672.13$

1 By the terms of the sealing convention of July 7, 1911, Japan and Great Britain are each entitled to 15 per cent of the annual take of fur-seal skins on the Pribilof Islands. If 15 per cent of the total annual take does not equal 1,030 skins, then each country is to receive 1,000 skins. 


\section{XXII.}

ITemorandum statement regarding 6,200 commercial sealskins taken on the Pribilof Islands, Alaska, in calendar years 1917 and 1918 and sold at Public auction A prit 28, 1919. ${ }^{1}$

Gross amount of sale.

$\$ 455,275.00$

Expenses of sale, transportation, etc.:

Cash discount, 1 per cent................... \$4,552.75

Commission, $3 \frac{1}{2}$ per cent................... 15,775. 28

Dressing, dyeing, and machining............... ${ }^{2} 85,240.75$

Transit insurance on 6,220 skins, at $\$ 0.135 \ldots \ldots \ldots \ldots . \quad 839.70$

Freight on 6,220 skins, at $\$ 0.19$ each............... ${ }^{3} 1,181.80$

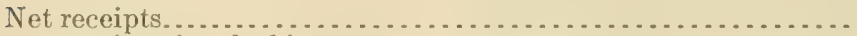

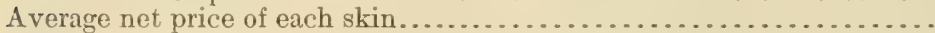

Great Britain's share of 1,000 skins for 1917

$55,900,00$

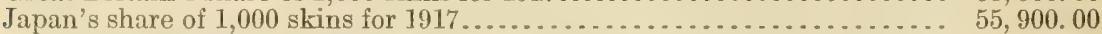

Great Britain's share of 4,277 skins taken in 1918, 15 per cent of $4,277 \times$

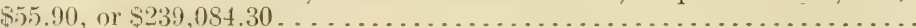

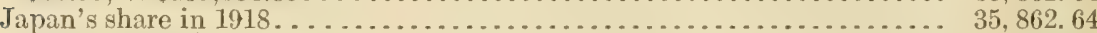

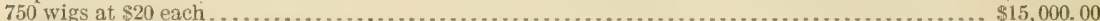

1,905 extra extru lárge, at $\$ 19$ each. . . . . . . . . . . . . . . . . . . . . . . . . . . . . . . . . . . . . . .

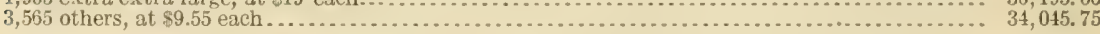

\section{XXIII.}

$85,240.75$

Hemorandum stalement regarding 9,055 commercial sealskins taken on the Pribilof Islands, Alaska, in calendar year 1918 and sold at public auction September 10, 1919.

Gross amount of sale.

$\$ 827,112.50$

Fxpenses of sale, transportation, etc.

Cash discount............................ \$8,271. 12

Commission of agent $3 \frac{1}{2}$ per cent................... 28,659.45

Dressing, dyeing, and machining................. $108,253.95$

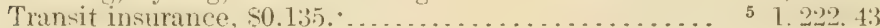

Freight on 9,055 slins at $\$ 0.19$ each $\left.\ldots \ldots \ldots \ldots \ldots \ldots .{ }^{6}\right], 720.45$

148. 127.40

Net receipts.

$678,985.10$ 743 wigs at $\$ 20$ each

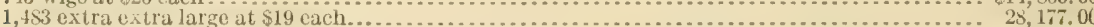

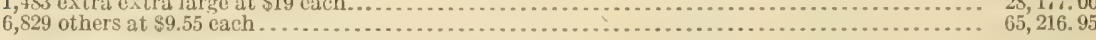

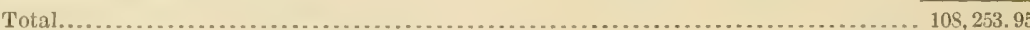

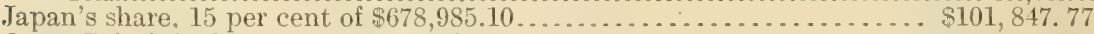

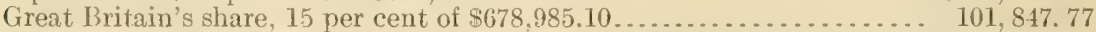

1 This sale contained 10,102 skins as follows: 3,882 food skins and 6,220 commercial skins. The commercial shins consisted of 1,943 taken in the caleniar year 1917 and 4,277 taken in the calenkar year 1918 . The foreign countries had no financial interest in the food skins.

2 This is the actual amount paid for dressing, dyeing, and machining the sealskins at prices given in supple mental contract with Funsten Bros. \& Co:

3 The unit cost of $\$ 0.19$ per skin for transportation is determined by the payment of $\$ 3,395.63$ on 17,816 shins shipped from Seattle to St. Louis on October 5, 1918.

4 This is the antual amount paid for dressing, dyeing, and machining the sealskins at prices given in supplemental contract with Funsten Bros. \& Co,

5 The unit cost of $\$ 0.135$ per skin for transit insurance is determined by the premium of $\$ 2,405.25$ paid on 17,816 skins from Pribilof Islands in September, 1918.

6 The unit cost of $\$ 0.19$ per skin for transportation is determined by the payment of $\$ 3,395.63$ on 17,816 skins shipped from Seattle to St. Louis on October 5, 1918. 


\section{XXY}

Memorandum statement regarding 9,100 sealskins taken on the Pribilof Islands, Alaska, in calendar year 1918 and sold at public aucition Februury 2, 19:0.

Gross amount of sale

Expenses of sale, transportation, etc.:

Cash discount 1 per cent.................. \$12,829.05

Commission. $3 !$ per cent.................. 44, 452. 66

Dressing, dyeing, and machining............... ${ }^{1} 118,322.90$

Freight on 9,100 skins at $\$ 0.20$ each............. ${ }^{2} 1,820.00$

Net receipts............................. $1,105,180.39$

Creat Britain's share, 15 per cent of $\$ 1,106,480.39 \ldots \ldots \ldots \ldots \ldots \ldots \ldots . \$ 165,822.06$

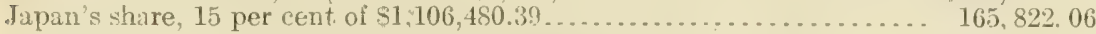

XXV.

Memorandum statement regarding 5.740 commercial sealskins taken on Pribilof Istands, Alaska, in calendar year 1918, and sold at public auction May 10, $19200^{3}$

Gross amount of sale

$\$ 423,530.00$

Expenses of sale, transportation, etc.:

Cash discount....................... \$4,235.30

Commission............................... 14,675.32

Dressing, dyeing, and machining................ ${ }^{4} 71,465.85$

Freight on 5,740 skins, at 20 cents each............. ${ }^{5} 1,148.00$

Net receipts

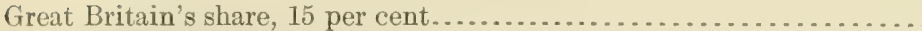

\section{XXVI.}

Memorandum statement regarding 10,120 senlskins taken on the Pribilof Islands. Aluska, in calendar years 1918 and 1919, and sold at public auction February 21, 1921.

Gross amount of sale of 6,690 skins taken in 1918 .

$\$ 230,079.00$

Expenses of sale:

Cash discount..................... \$2,300.79

Commission, $3 \frac{1}{2}$ per cent................. 7,972.24

Freight on 6,690 skins, at 23 cents each $\ldots .{ }^{6} 1,538.70$

Total

1 This is the actual amount paid for dressing, dyeing, and machining the sealskins at prices given in supplemental contract with Funsten Bros. \& Co.:

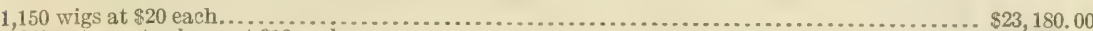

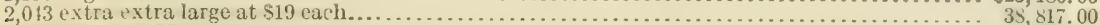

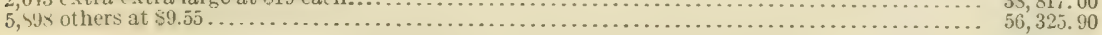

Total.

$118,322.90$

2 The unit cost of $\$ 0.20$ per skin for transportation is determined by the payment of $\$ 7,049.35$ on 34,831 skins taken in the calendar year 1918, and shipped from Seattle, Wash., to St. Louis, Mro., in 1918 and 1919.

3 This sale con isted of 5,752 skins, of which number 12 were lood skins and the foreign countries had no financial in terest therein.

${ }_{1}$ This is tine actual amount paid for dressing, dyeing, and machining the sealskins at prices given in supplemental contract with Funsten Bros. \& Co.

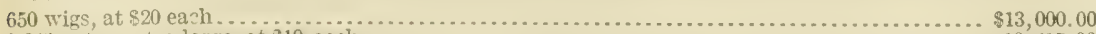

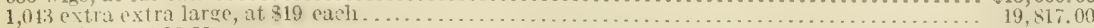

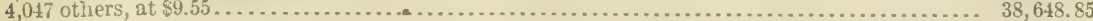

$71,465.85$

5 The unit cost of $\$ 0.20$ per skin for transportation is determined by the payment of $\$ 7,049.35$ on 34,831 skins taken in the calendar year 1918 and shipped from Seattle, Wash., to St. Louis M., in 1918 and 1919.

6 The unit cost of 23 cents per skin for transportation is determined by the payment of $\$ 5,848.77$ on 25,238 skins shipped from Seattle, Wash., to St. Louis, Mo., on Oct. 27, 1919.

S. Doc. $213,67-2-3$ 
Gross amount of sale of 3,430 skins taken in 1919 . $\$ 125,610.00$

Expenses of sale:

Cash discount.................... $\$ 1,256.10$

Commission, $3 \frac{3}{4}$ per cent ................. 4,663. 27

Freight on 3,430 skins, at 23 cents each... $\quad{ }^{4} 788.90$

6,708.27

Total $\$ 118,901.73$

Grand total................................. 327, 169.00

Reimbursement on $\$ 200,000$ paid to Funsten Bros. \& Co., in lieu of expenses incurred in storing, dressing, dyeing, and machining Government sealskins as per contract with Fouke Co., dated Feb. 15, 1921 .

Net receipts $125,000.00$ $212,169.00$

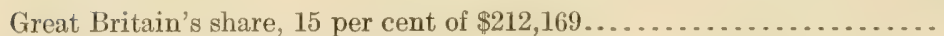

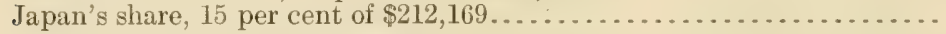

\section{XXVII.}

June $7,1921$.

Dear Sir: There is inclosed herewith for delivery to the Japanese ambassador official check No. 1440, dated June 7, 1921, and drawn to the order of the Government of Japan, in the sum of $\$ 231.92$.

This check represents 15 per cent of the net proceeds of the sale of 14 sealsking taken on the Pribilof Islands, Alaska, during the calendar years 1918 and 1919, and sold by this department to the Fouke Fur Co., of St. Louis, Mio., to be used by them for exhibition purposes. The department received the sum of $\$ 1,546.16$ for these skins, at the rate of $\$ 35.14$ per skin, which is the average price established at the sale of February 21, 1921.

Yours very truly,

The Secretary of State.

C. H. Huston, Assistant Secretary of Commerce.

\section{XXVIII.}

Memorandum statement regarding 10,060 scalskins taken on the Pribilof Islands, Alaska, in calendar year 1919 and sold at public auction May 23, 1921.

Gross amount of sale of 10,060 skins taken in 1919 . $\$ 359,715.00$

Expenses of sale:

Cash discount, 1 per cent..............\$3, 597.15

Commission, $3 \frac{3}{4}$ per cent.................. 13, 354.42

Freight on 10,060 skins, at $\$ 0.23^{1}$ each..... 2,313. 80

$19,265.37$

Total......................................... $\$ 340,449.63$

Reimbursement on $\$ 200,000$ paid to Funsten Bros. \& Co. in

lieu of expenses incurred in storing, dressing, dyeing, and machining Government sealskins as per contract with Fouke Co., dated Feb. 16, 1921....................

Dressing, dyeing, and machining 4,180 skins, at $\$ 16.50$ per

skin.

$68,970.00$

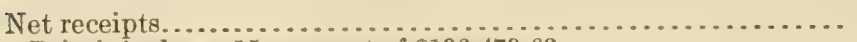

Great Britain's share, 15 per cent of $\$ 196,479.63 . \ldots \ldots \ldots \ldots \ldots . . . . . .$.

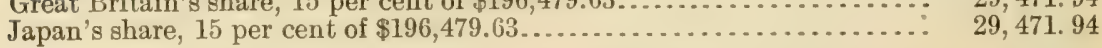

$143,970.00$

Whereas the Government of the United States is the owner and has under its control certain sealskins and certain fox skins, taken annually on the Pribilof Islands and in Alaska, the taking and disposal of which are, subject to existing laws, under the control of the Secretary of Commerce: and

1 The unit cost of 23 cents per skin for transportation is determined by the payment of $\$ 5,848.77$ on 25,238 skins shipped from Seattle, Wash., to St. Louis, Mo., on Oct. $27,1919$. 
Whereas Funsten Bros. \& Co., a corporation created by and existing under the laws of the State of Illinois, and having its principal office located at Second and Elm Streets, rity of St. Louis, State of Missouri, is engaged in the business of storing, sorting, and selling fur skins; and

Whereas the corporation mentioned satisfactorily conducted for the Government the sale of all skins taken by it from the Pribilof Islands and in Alaska during the year 1913; and

Whereas it is the desire of the Secretary of Commerce to continue the sales of such skins in the United States, in order to establish therein a permanent sealskin and foxskin market, and also to establish in the United States the industry of dyeing and dressing such skins:

Now, therefore, this agreement, made and entered into this 30th day of August, $1915, b y$ and between the United States of America (hereinafter designated as the Government), by the Acting Secretary of Commerce (in accordance with and by virtue of authority conferrel by the act of April 21, 1910, 36 Stat. 326, and the act of A ugust 24, 1912, 37 Stat. 499), of the first part, and Funsten I3ros. \& ('o. (hereinafter designated as the company), a corporation as aforesaid, of the second part:

Witnesseth, that for and in consideration of the covenants and agreements herein. after stated, the Government will ship to the company, at St. Louis. Mo., such sealskins and fox skins as are taken annually by it at the Pribilof Islands and in Alaska, and are available for sale by the (iovernment, and the company will receive and accept the same at said city of St. Louis, Mo., as a consignment from the Government, to be sold by the company at public auction, or to have dressed and dyed and then sold by the company at its salesrooms in the city of St. Louis, on behalf of the Government upon the terms and conditions following, that is to say:

First. All freight charges and other expenses involved in the transportation of said sealskins and said fox skins from San Francisco, Calif., Seattle, Wash., or other port to which they may be brought, to the warehouse of the company in St. Louis, incluling advance charges at San Francisco, Calif., Seattle, Wash., or other port to which they may be brought, and carriage at St. Louis, shall be paid by the company and deducted from the proceeds of sales, as hereinafter provided.

Second. Insurance upon said sealskins and fox skins for the benefit of the Government, covering loss or damage thereto on the voyage from the Pribilof Islands to St. Louis, Mo., or any part of such voyage, in such amount as shall be satisfactory to the Secretary of ('ommerce, shall be taken out (if requested by the Government) by the company and the premium or premiums for such insurance shall be paid by the company and deducted from the proceeds of sales, as hereinafter provided.

Third. After the receipt of said sealskins and said fox skins at the warehouse of the company in St. Louis, they shall be properly assorted according to size, quality, and condition, in conformity with custom, and shall be placed in cold storage and kept therein until exhibited for sale and delivery to buyers; and a copy of the assortment so marle shall be transmitted by mail to the Secretary of Commerce as soon as possible after the same is completed. While in the possession of the company in the city of St. Louis, said seal and fox skins shall be kept insured by them against loss by fire, and all due and customary precautions shall be taken by the company for the safekeeping of said skins and to prevent deterioration, the expenses thereof being borne by the company.

Fourth. Said sealskins and said fox skins shall be sold by the company at public auction at its salesrooms in the city of St. Louis, Mo., on the customary terms and at such time or times as shall be deemed most advantageous to the Government. The company shall promptly advise the Secretary of Commerce of the time when it proposes to make such sale, and the Secretary of Commerce shall have the right, not less than 15 days before the time at which said sale is appointed to be held, to change the time and determine when such sale shall occur. At such sale or sales the Secretary of Commerce, or his duly authorized representative, shall have the right to withdraw from sale said sealskins and said fox skins or any lot or lots thereof, at any time before the same shall be actually sold.

Fifth. The company shall render to the Secretary of Commerce just and proper accounts of the gross proceeds of any and all sales of said sealskins and said fox skins affected by it, and of all sums received and paid by it; and will keep such accounts in proper books which shall be deemed the property of the Government. And the Secretary of Commerce shall at all times be at liberty, either personally or by agent duly authorized by him, to inspect and carry away such books, or to make and carry away copies of or extracts from such books.

Sixth. The company shall remit and pay to the Secretary of Commerce the net proceeds of any and all sales of said seal and fox skins effected by it, such net proceeds 
to be determineri as hereinafter provided, within one month after such sales shall have occurred.

Seventh. In making up the account of the sales of said sealskins and said fox skins, and in order to ascertain the net procests thereof, the company shall be entitled to receive and retain out of the cross proceeds of said sales the commission hereinafter specified as and for full compensation for all services it may render and all expenses it may incur in connection with the storage, sorting, protestion, insurance as aforesaid, and proper (are of all of said skins and the advertising and sale thereof, and shall also deduct all costs and expenses incidental to transportation and all expenses for insuranee during transportation, if any have heen incurred, hereinbefore mentioned, and the amount remaining subject to provisions hereinater stated shall be the net proceets of said sales, and payahle to the secretary of ('ommerce. The company shall be entitled to deduct the amount expended for cold storage charge for the period in excess of six months, on the skine ortered held by the secretary of commerce from the year 1913 and the year 191.4 up to the time said skins are either dressed and dyerd or sold, surch charge to be computed at regular market rates, and whenever the Government shall order skins held ior a period longer than six months, then the romnany shall also be entitled to dexluct the amount expended for cold storage charge for the period in excess of said six months on the skins so ordered held.

Eighth. All casks in which suid sealskins and fox skins are parked, and all surplus silt, which may come from sail skins, shall, if the same has a salable value be sold by the company and the inll amount of the proreests shall be accomed for and paid over by the company to the sincretary of commerce when set tlement for the proceeds of the sale of said skins shall be made.

Ninth. All contrates made by the company relating to said sealskins and said fox skins for their transportation or their care and proter tion pending sale, and for the sale and delivery thereof, and all contracts or policies of insurance against risks of transportation, fire, and otherwise may he made hy the company in its own name, but the same shatl inure to the henefit of the (iovernment: and all claims for loss of said sealskins and said fox skins, or any or either of them, or for damage thereto, which the company may be entitled to make or shall collect against common carriers: or loss or damage by reason of failure to safely and duly transport and deliver such skins, shall likewise inure to the benetit of and he paid to the ciovernment, less the lemal charge for collection.

Tenth. Huring the closed season period in which the killing of seals on the Pribilot Islands is restricted to the number recpuired to supply food, clothing, and hoat skins for the natives on the islands, as provided by the act of . ugrust 24,1912 ( that is to say, during the years 1915, 1916, and 1917, unless such closed season should be sooner terminated by law), the (onmission of 4 per cent shall be paid to the company as and for full compensation for all services it mayrender and all expenses it may incur in connection with the storage (exrept skins ordered held longer than six months), sorting, proterting, insurance as aforesid, and proper "are of all of said skins, and the advertising and sale thereof. Cpon the expiration of said closed season, under the law as it now stands, or as it may be hereafter amended or changed, such commission shall be as follows:

1. If the total number of sealskins and fox skins combined to be sold, ronsigned in any one year to the company, shatl not exceed 20,000 , the rate of commission figured on the basis aforesaid shall be 4 per cent.

2 . If such total number shall exceed 20,000, but not exceed 30,000 , the rate of such commission shall be $3 \frac{3}{4}$ per cent.

3. If such total number shall exceed 30,000 , hut not exceed 40,000 , the rate of such commission shall be $3 \frac{1}{2}$ per cent.

4. If such total number shall exceed 40,000 , but not exceed 50,000 , the rate of such commission shall he $3 \frac{1}{\text { per cent. }}$

5. If such total number shall exceed 50,000 , the rate of such commission shall be 3 per cent.

If at any time either the Government or the company shall be dissatisfied with the foresoing vrovision with respert to the enmmiseion to be paid atter the expiration of said closed season. written notice thereof shall be given to the other parties hereto, and

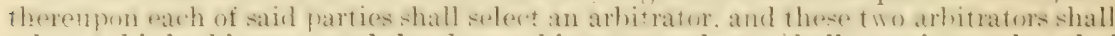
select a third arbitrator; and the three arbitrators so chosen shall constitute a board of arbitration which shall have' full authority to determine whether the comnission herein provided is just and fair und, if they deem it necessary, to readjust it as they may see lit; and both of the parties hereto agree to abide by the decision of such arbitrators.

Eleventh. In consideration of the premises, and especially of the compensation provirted for in this agreement, the company hereby agrees to bring to the United 
States fortheith. as soon as arranements can he completed and establish therein a plant using what is commonly known as the lice procese of dresing and dyeing sealskins. or one using a dressing and dyeing process satisfactory to the secretary of Commeree it being understesd and arreed that the dressing and dyeing company, when reduested so to do, will dress and dye sealskins in such plant for the (iovernment and for individuals, and that the charges to the (iovernment as well as to individuals for dres-ine and dreing seallsins and foy skins in such plant shall not ex. eed the charges heretoinre mar!e in L.endon, Eneland, escept in sn far as may be incident to necessary increased cost of operation. 'I he said company is hereby authorized and directed to arrange bor such dressing and dreing plant to proreed as som as posithle to dress and dye in the usual manner the sealskins of the Government now in possession of said company amounting to about 3,280 skins, and also to make like arrangements for the dressing and dreing of the sealskins which are taken in the year 1915, anounting to ahout 5,000, which are expected to arrive by September, 1915. For the dressing and dyeing of all said skins the contpany arees that the cost to the (iovernment shall be ss per skin, hased on the chare heretofore marle in I ondon. Fngland, for dreesing and dyeines skins hy the Jiere dresing and dyeiner plant, in addition to the compensation hereinhefore provided for. and the company is herehy authorized and directed to retain from the proceeds of the sale of any and all skins sold by the company for aceount of the foremment the said ss per skin, which the company arrees lo ad vance sand pay to the said plant for dressing and dreing said sealskins.

Twelith. Should the company hail to establish therein a plant using said process, or one using a dressing and dyeing process satisfactory to the said secretary of ( ommerer, within the period specifed in section 11 hereof, or if at any time during the continuance of this contract the company shall fail to faithfully perform the terms and conditions therenf on its part to be kept and performed, the Government may forthwith terminate the same.

Thirteenth. If at any time during the continuance of this contract the Government shall derm it for its hest interests that for any year or years the sale of the said sealskins or said fox skins, or either or any of then, shall be held in London, Fingland, the company, upun receiving reasonable notice thereof from the secretary of $($ ommerce, shall catise all steps to be taken necessary to full compliance with the directions of the

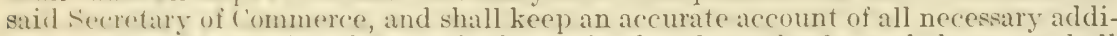
tional expenses ocrasioned by such change in the place of sale, and the same shall consitute a proper charge against the Government and shall be deducted from the gross proceeds of such sale.

Finteenth. Tnless sooner terminated as hereinbefore provided, this contract shall continue in force and effect until the 1st day of January, 1921.

Fifrenth. It is understood and agreed by the parties hereto that this contract and all the provisions thereof shall be subject to any legislation that may hereafter be enacterl by ('ongress relative to the subject matter hereof, and also to such rules and regulations ats may be adopted, from time to time, by the secretary of commerce.

Sixteenth. Pursuant to section 3741 of the Revised Statutes of the United States, it is further agreel that no Member of or Delegate to C'ongress, or Resident ('ommissioner or any officer or employee of the Government or other person whose name is not at this time disclosed, shall be admitted to any share or interest in this contract, or to :ny henetit to arise therefrom; and that this contrart shall be subject in all respects to the provisions of sections 114, 115, and 116 of the Criminal Code of the United States, so far as the same may be applicable.

serenteenth. The covenants and agreements, and earh of them herein rontained, shall not be binding on the company if prevented by fire, death, strikes, the act of God, or any other unavoidable cause not within the control of the company.

Signed in duplicate by the parties hereto the day and year first above written.

[SEAL.]

United States of America, By E. F. SWEET,

Acting Secretary of Commerce.

Funsten Bros. \& Co.,

By P. B. Fouke,

President.

[CORPORATE SEAL.]

A. E. Murphy,

Acting Secretary.

Signed, sealed and delivered in the presence of-

DANL. J. Richardson.

Virgil RULe. 
The corrections in the foregoing agreement, set forth below, were marle before the execution of the contract:

Page 1, line 21, the name "William C. Redfield" stricken out and the words "the Acting" interlined.

Page 2, line 17, parentheses placed around the words "if requested by the Government."

Page 4, line 7, the word "a" changed to "the."

Page 6, the words "dressing and dyeing" inserted between the words "the" and "company" on line 17, and the words "based on" inserted between the parentheses and the word "the" on line 31.

I, A. E. Murphy, do hereby certify that I reside at St. Louis, Mo., and am the acting secretary of Funsten Bros. \& Co., the corporation described in and which executed the foregoing contract, and that as such I have custody of its records and seal; that the said corporation is organized under the laws of the State of Illinois; that the corporate seal affixed to said instrument is the seal of said corporation and was thereto affixed by order of its board of directors; that P. B. Fouke is the president of said corporation and was duly anthorized to execute said instrument in its behalf; that I know the signature of said officer, and that the signature affixed to such instruments is his signature; that the following is a true and correct copy of so much of the by-laws or resolutions of said company or its board of directors as confers authority to execute said contract upon the said officer who executed them:

SEC. 7 (Subsec. 1). Duties of president.-The president shall execute all bonds, contracts, or other instruments requiring to be made or executed for and on behalf of the company; he shall sign all bills, notes, and drafts, and indorse same when required by the needs of the business. He shall also have the power to sign checks and indorse same."

In witness whereof I have hereunto set my hand and affixed the seal of said corporation at St. Louis this 30th day of August, 1915.

(Affix corporate seal.)

A. E. MurPhy, Acting Secretary.

\section{SUPPLEMENTAL AGREEMENT.}

Whereas the parties hereto, the Government of the United States, by Edwin F. Sweet, Acting Secretary of Commerce, and Funsten Bros. \& Co, a corporation created and existing under the laws of the State of Illinois, made and entered into a certain written agreement dated August 30, 1915, under the terms of which the Government agreed to ship to the company, at St. Louis, Mo., such sealskins and fox skins as are taken annually by it at the Pribilof Islands and in Alaska and are available for sale by the Government, and the company agreed to receive and accept the same at said city of St. Iouis, Mo., as a consignment from the Government, to be sold by the company at public auction, or to have dressed and dyed and then sold by the company at its salesrooms in the city of St. Louis, on behalf of the Government, upon the terms and conditions in said written agreement contained.

And whereas section 11 of the said written agreement provided in part as follows:

"In consideration of the premises, and especially of the compensation provided for in this agreement, the company hereby agrees to bring to the United States, forth with as soon as arrangements can be completed, and establish therein a plant using what is commonly known as the Rice process of dressing and dyeing sealskins, or one using a dressing and dyeing process satisfactory to the Secretary of Commerce; it being understood and agreed that the dressing and dyeing company, when requested so to do, will dress and dye sealskins in such plant for the Government and for individuals, and that the charges to the Government as well as to inclividuals for dressing and dyeing sealskins and fox skins in such plant shall not exceed the charges heretofore made in London, England, except in so far as may be incident to necessary increased cost of operation."

And whereas the said Funsten Bros. \& Co. have faithfully carried out the above quoted portion of the said section 11 of the said written agreement.

And whereas it is now the desire of the Secretary of commerce to enter into an agreement with the said Funsten Bros. \& Co., supplemental to the said agreement of $\Lambda$ ugust 30 . 1915, setting forth the prices to be paid by the Government for dressing and dyeing skins in said plant for the period from 1917 to 1922, and for other purposes:

Now, therefore, this supplemental agreement made and entered into this $3 \mathrm{~d}$ day of July, 1917, by and between the United States of America, hereinafter designated as the Government, by E. F. Sweet, Acting Secretary of Commerce (in accordance with 
and hy rirtue of the anthority conferred by the act of April 21. 1910 (36. Stat. 326), and the act of August 24, 1912 (37 Stat. 499), of the first part, and Funsten Bros. \& Co., hereinafter designated as the company, a corporation, as aforesaid. of the second part.

Iritnesseth, that for and in consideration of the mutual promises and agreements herein contained the said parties hereby covenant and agree as follows:

First. That during the term of this supplemental contract all sealskins taken annually by the (Govermment at the Pribilof Islands and in Alaska that are available for sale by the Government, other than "wigs" (i. e., the skins of large males or bulls) shipped to the rompany hy the Government under the terms and provisions of the said original contract. shall be dressed. dyed, and machined by the company at its dressing and dyeing plant in St. Louis, Mo., and all wigs shall be washed and dried or dressed or otherwise treated as directed by the Government.

second. That the company will dress, dre, and machine at its said plant all skins. other than wigs, shipped to it by the Government, using therefor the process which it has heretofore used and is now using, and will handle all wigs received as direrted by the Government, the company's charges for such services to be as set forth herein, which cliarges the company is herebv anthorized and directert to retain from the proceeds of the sale of any and all such skins sold by the company for account of the Government; it being understood and agreed that each and all of the provisions, stipulations, and conditions of the said original agreement of August 30. 1915) (exrept (late of expiration). relating to the sale of sealslins and fox skins for the (rovermment, shall be and are hereby made a part of this supplemental agreement, and are made applicalile to the sale of wigs and of skins dressed, dyed, and machined as herein provided:

Prices for washing, drying, dressing, etc., of wigs, or the skins of large males or

bulls, per annum:

Washed and dried.

Each.

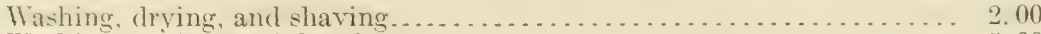

Washing, drying, and dressing............................. 5.00

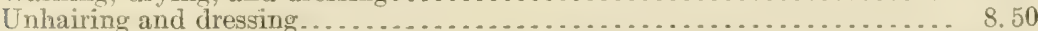

All other sealskins to be dressed, dyed, and machined at the following prices:

15,000 or less per annum. ............................. $\$ 10.00$

Exceeding 15,000 but not exceeding 20,000 per annum . . . . . . . . . . 9.75

Exceeding 20,000 but not exceeding 25,000 per annum . . . . . . . . . . 9.70

Exceeding 25,000 but not exceeding 30,000 per annum. . . . . . . . . . . 9. 65

Exceeding 30,000 but not exceeding 35,000 per annum . . . . . . . . . . . 9.55

Exceeding 35,000 but not exceeding 40,000 per annum . . . . . . . . . . . . 9.35

Exceeding 40,000 but not exceeding 45,000 per annum . . . . . . . . . . . 9. 25

Exceeding 45,000 but not exceeding 50,000 per annum. . . . . . . . . . . 9.00

Exceeding 50,000 per annum.............................. 8.90

Thirl. That the company will, during the term of this supplemental agreement. at its own expense and without any cost or charge whatsoever to the Government, by inserting advertisements in the proper technical press, and by circulating. distrihuting, and mailing circulars, bulletins, letters, etc., and by other proper methods, advertise the quality and adrantages of sealskins dressed and dyed by the said process, with the view of informing the public as to the merits of such process and of inducing a more general use of sealskins than prevails at the present time.

Fourth. That the company will, during the term of this supplemental contract, during the fur-seal killing season each year, as the Government may direct, send to the Pribilof Islands two or more experts experienced in the killing and skinning of seals and the salting of sealskins, for the purpose of cooperating with the departments representatives and of instructing and assisting the natives and others engaged in such work, in order that skins taken by the Government may be secured and handled in the best possible manner.

Fifth. That the salaries and compensation of such experts, as well as all other expenses incident to their employment, including traveling expenses, subsistence, etc., shall be borne by the said company, provided, however, that the Government shall, without expense to the company, transport said experts, from some port on the Pacific coast, to be designated by the Government, to and from the said islands on vessels owned or leased by the Government, and shall furnish said experts with board and lodging while being so transported and while on the said Pribilof Islands for the purposes aforesaid.

Sixth. That the company will, during the term of this supplemental contract, on or before the Ist day of May in each year, advance to a representative of the Govern- 
ment, to be designated by the Secretary of Commerce, such sum as shall be determined by the (iovernment to be necessary to enable its representative to pay such compensation for services as shall be fixed by said Secretary of ('ommerce to persons engaged in killing and skinning seals, and salting and handling sealskins, or otherwise emploved in connection therewith preparatory to the sale of said skins, it heing understood and agreed that the company will deduct from the proceeds of the sale of skins the advances made by it as aforesaid for the payment of any services rendered in connection with the particular skins included in any sale.

Seventh. That when the said wigs shall have been treated as directed by the Government and when other sealskins shall have been dressed, dyed, and marhined as herein provided for, and while in the possession of the company thereafter, they shall be insured against loss by fire, and all due and customary precautions shall be taken by the company for the safeguarding of all of the said skins, the expenses thereof to be borne hy the company. The insurance herein provided for is to be in addition to that provided for in section 3 of the original contract of August 30, 1915, and shall he based upon the increased value of such skins by reason of such dressing and dyeing, as aforesaid.

Eighth. It is understood and agreed by the parties hereto that this supplemental contract, and all the provisions thereof, shall be subject to any legislation that may hereafter be enacted by Congress relative to the subject matter hereof, and also to such rules and regulations as may be adopted from time to time by the Secretary of Commerce.

Ninth. Pursuant to section 3741 of the Revised Statutes of the United States, it is further agreed that no Member of or Delegrate to ('ongress, or a Resident ('ommissioner, or any oflicer or employee of the Government, or other person whose name is not at this time disclosed, shall be admitted to any share or interest in this contrart, or to any benefit to arise therefrom, and that this supplemental contract shall be subject in all respects to the provisions of sections 114,11 ), and 116 of the ('riminal ('ode of the United States so far as the same may be applicable.

Tenth. That unless sooner terminated, this supplemental contract shall continue in force and effect until the 1st day of January, 1922.

Eleventh. That the parties hereto hereby expressly agree that the said original contract dated August 30, 1915, for the sale of sealskins and fox skins shall be, and the same is hereby, extended in all its provisions to the 1st day of January, 1922.

Twelfth. That the parties hereto further covenant and arree that nothing contained herein shall be construed as altering, afiecting, or modifying any of the provisions, terms, or conditions of the said original contract not herein expressly altered, affected, or modified.

Signed in duplicate by the parties hereto this 3d day of July, 1917.

\section{[SEAL.]}

[CORPORATE SEAL.]

Witnesses:

R. L. Stancill.

G. Roscoe Firtich.

As to E. F. Sweet.

A. Murphy.

J. P. RANDALL.

As to Funsten Bros. \& Co.
United States of Anerica, By E. F. SWEET, Acting Secretary of Commerce.

Funsten Bros. \& Co.,

By P. B. Fouke, President.

D. J. Halley, Secretary.

SUPPLEMENTAL AGREEMENT.

Whereas, the parties hereto, the forernment of the Inited States. hy Edwin $\mathrm{F}$. Sweet. Acting sieretary of ('ommerce. and Funsten Bros. \& ('o.., a corporation created and existing under the laws of the State of Illinois, made and entered into a certain written agreement dated August:30. 1915, under the terms of which the Ciovernment agreed to ship to the company at St. Touis, Mo.. such sealskins and for skins as are annually taken by it at the Prihilof Islands and in . laska and are available for sale by the Government, and the company agreed to receive and accept the same at the city of $S t$. I ouis. No.. as a ronsignment from the Govermment, to he sold hy the company at public auction. or to have said sealskins and fox skins dressed and dyed and 
then sold hy the company at its salesrooms in the city of St. Louis, on hehalf of the Goremment, upon the terms and conditions in said written agreement contained.

Ind whereas the parties hereto. the Government of the United States, hy Edwin F. Sireet. Leting secretary of commerce, and Funsten Bros. \& ('o.. a corporation created and existing muler the laws of the State of Illinois, made and entered into a certain writen supplenental agreement to the said agreement of $A$ ugust 30.1915 , said supplemental agreement heing dated July 3. 1917, under the terms of which all the sealskins taken annually hy the Government at the Pribilof Islands and arailahle for sale hy the (iovermment shipped to the company by the fovernment under the terms and provisions of said original contract of August 30, 1915), should he dressed, dyerl, and machined lyy the company at its dressing and dyeing plant in sit. I.ouis Mo., at and for the prices set out in said supplemental agreement of July $3,1917$.

And whereas the said Funsten Bros. \& Co. have faithfully kept and performed the terms and conditions of the said original agreement of $\$$ ugust 30.1915 , and of the said supplemental agreement of July 3,1917 , on its part to be kept and performed.

And whereas it is now the desire of the Secretary of Commerce to enter into an acreement with the said Funsten Bros. \& (o., supplemental to the said agreement of August : $0,191 \bar{i}$, and to the supplemental agreement of July 3, 1917, extending the term of the said arrement of Aurust 30,1915 , and of the said supplemental agreement of July 3. 1917, and providing for certain changes and alterations in some of the terms and conditions thereof:

Now, there, this supplemental agreement made and entered into this 20 th day of June, 191!, by and between the [nited States of America, hereinafter designated as the (iovernment, by Elwin F. Sweet, Acting Secretary of fommerce in accortance with and hy virtue of the authority conferred by the act of $A$ pril 21,1910 (36 Stat. $32(i)$, and the aret of August 24, 1912 (37 Stat. 499), of the first part, and Funsten Bros. E ('o., herunafter lesignated as the company, a corporation as aforesaid, of the second part,

Witnesseth, that for and in consideration of the mutual promises and agreements herein contained, the said parties hereby covenant and agree as follows:

First. That paragraph 2 of the said original written agreement of August 30,1915 , be and the same is hereby revoked, and that from the date of this supplemental agreement insurance as therein provided for shall not be carried.

second. That paragraph 6 of the said original witten agreement of August 30,1915 , be and the same is hereby revoked, and the following substituted therefor:

"The company shall remit and pay to the secretary of Commerce the net proceeds of any and all siles of said seal and fox skins effected by it, such net proceeds to be deternined as hereinaiter provided, within 45 days after such sales shall have occurred.'

Thirl. That paragraph 6 of the said supplemental agreement of July 3, 1917, be and the same is hereby revoked, and the following substituted therefor:

"That the company will, during the term of this supplemental contract, on or before the lst day of May in each year, advance to a representative of the Government to hr desicnated hy the secretary of Commerce such sum as shall be determined by the ciovermment to he neressary to enable its representatives to pay such compensation for services as shall be fixed by the said secretary of Commerce to persons encaged in killing and slimning seals and salting and handling sealskins, or otherwise employed in connection therewith, preparatory to the sale of said skins, it leing understoorl and arreed that the commany will dedurt from the proceeds of the first sale of the skins following the annual sealing operations the adrances made hy it as aforesaid."

Fourth. It is understood and agreed by the parties hereto that the company will, on the redultest of the fierretary of Commerce, assume and pay all necessary expenses for supplies, matrials, including salt, casks, twine, and other necessaries, in the taking, curing. handline, and shipment of seal and fox skins, and will deduct from the prorecels of the limst sale of skins following the annual sealing operations the payments made by it as aforesaid.

Fifth. That the lirst paragraph of the supplemental agreement of July 3,1917 , be and the same is hereby revoked, and the following substituted therefor:

"That during the term of this supplemental agreement all sealskins taken annually b) the Government on the Prihilof Islands and in Alaska, that are available for sale by the (iovernment, shipped to the company by the Govermment under the terms and provisions of the said original contract of 11 gust $: 0,1915$, shall he dressed, dyed, and machined by the company in St. Louis, Mo.

Sixth. That paragraph 2 of the sail supplemental agreement of July 3,1917 , he and the same is hereby revoked, and the following substituted therefor:

"That the company will dress, dye, and machine at its said plant all skins shipped to it by the Government, using therefor the processes which it is now using or any 
process which may be an improvement on the process it is now using, the company's charges for such services to be as set forth herein, which charges the company is hereby authorized and directed to retain from the proceeds of the sale of any and all such skins sold by the company for account of the Government, it being understood and agreed that each and all of the provisions, stipulations, and conditions of the said original agreement of August 30, 1915, except the date of expiration, relating to the sale of the sealskins and fox skins for the Government, shall be and are hereby made a part of this supplemental agreement, and are made applicable to the sale of the skins dressed, dyed, and machined as herein provided.

'Sealskins will be dressed, dyed, and machined by the company at the following graduated prices, which are based on the total number of skins taken by the Government each calendar year. Included in such number and for such prices are the skins credited to or belonging to the Governments of Great Britain and Japan, under the convention of July $7,1911$.

15,000 or less per annum . . ............

Exceeding 15,000 but not exceeding 20,000 per annum...................... 9.75

Exceeding 20,000 but not exceeding 25,000 per annum......................... 9. 90

Exceeding 25,000 but not exceeding 30,000 per annum..................... 9.65.

Exceeding 30,000 but not exceeding 35,000 per annum..................... 9. 95

Exceeding 35,000 but not exceeding 40,000 per annum.................. 9.35

Exceeding 40,000 but not exceeding 45,000 per annum................. 9.25

Exceeding 45,000 but not exceeding 50,000 per annum................ 9. 00

Exceeding 50,000 per annum . . . . . . . . . . . . . . . 90

"The following special prices will be charged for the dressing, dyeing, machining," and finishing of skins of size ordinarily designated as "extra extra large" and "wigs," namely, for skins whose length when dressed and dyed equals 49 inches and does not. exceed 55 inches, $\$ 19$ per skin; and for skins whose length when dressed and dyed is over 55 inches, $\$ 20$ per skin."

Seventh. That paragraph 7 of the said supplemental agreement of July 3, 1917, be, and the same is hereby, revoked, and the following substituted therefor:

"That when sealskins shall have been dressed, dyed, and machined as herein provided for, and while in possession of the company thereafter, they shall be insured against loss by fire and all due and customary precautions shall be taken by the company for the safeguarding of all of the said skins, the expenses thereof to be borne by the company."

Eighth. It is understood and agreed by the parties hereto that this supplemental contract and all the provisions thereof shall be subject to any legislation that may hereafter be enacted by Congress relative to the subject matter hereof, and also to such rules and regulations as may be adopted from time to time by the Secretary of Commerce.

Ninth. Pursuant to section 3741 of the Revised Statutes of the United States, it is further agreed that no Member of or Delegate to Congress, or a Resident Commissioner, or any officer or employee of the Government, or other person whose name is not at this time disclosed, shall be admitted to any share or interest in this contract or to any benefit to arise therefrom, and that this supplemental contract shall be subject in all respects to the provisions of sections 114, 115, and 116 of the Criminal Code of the United States, so far as the same may be applicable.

Tenth. That, unless sooner terminated, this supplemental contract shall continue in force and effect until the 15th day of December, 1926.

Eleventh. That the parties hereto hereby expressly agree that the said original contract dated August 30, 1915, and the supplemental contract dated July 3, 1917, shall be and the same are hereby extended in all their provisions except as herein modified to the 15th day of December, 1926.

T'welf th. That the parties hereto further covenant and agree that nothing contained herein shall be construed as altering, affecting, or modifying any of the provisions, terms, or conditions of the said original contract of August 30,1915 , or of the said supplemental contract of July 3, 1917, not herein expressly altered, changed, or modified.

Signed in duplicate by the parties hereto this 20th day of June, 1919.

[SEAL.]

[CORPORATE SEAL.]
United States of America,

By EDwIN F. SWEET,

Acting Secretary of Commerce.

Funsten Bros. \& Co.,

By P. B. Fouke, President.

D. J. Halley, Secretary. 
I, D. J. Halley, do hereby certify that I reside at St. Louis, Mo, and am the secretary of Funston Bros. \& ( 0 ., the corporation described in and which executed the foregoing contract, and that as such I have custody of its records and seal; that the said corporation is organized under the laws of the State of Illinois; that the corporate seal affixed to said instrument is the seal of said corporation and was thereto affixed by order of its hoard of directors; that. I'. B. Fouke is the president of said corporation and was duly authorized to execute said instrument in its behalf; that 1 know the signature of said officer, and that the signature aftixed to such instruments is his signature; that the following is a true and correct copy of so much of the hy-laws or resolutions of sairl company or its board of directors as confers anthority to execute said contract, upon the said officer who executed them:

"SEc. 7 (Subsec. 1). Duties of president.-The president shall execute all bonds, contracts, or other instruments requiring to be made or executed for and on behalf of the company; he shall sign all bills, notes, and drafts, and indorse same when required by the needs of the business. He shall also have the power to sign cher.ks and indorse same."

In witness whereof I have hereunto set my hand and affixed the seal of said corporation at St. Louis, Mo., this 23d day of June, 1919.

(Affix corporate seal.)

$$
\text { D. J. Hallex, Secretary. }
$$

Whereas the Government of the United States is the owner and has and will have under its control certain sealskins and certain fox skins which have been or may be taken annually on the Pribilof Islands, the taking and disposal of whirh are in accordance with and subject to existing laws, under control of the Fecretary of commerce:

And whereas it appears to the satisfaction of the Secretary of Commerce that the financial condition of the Funsten Bros. \& ('o., a corporation organized and existing under the laws of the State of Illinois, which corporation has heretofore been employed as an agency for the sale of fox skins and the dyeing, dressing, machining. and sale of sealskins, is such as to jeopardize the interests of the Lnited States if permitted to further act as such agency;

And whereas the Fouke Fur Co., a corporation organized and existing under the laws of the state of Delaware, is authorized to conduct the business of storing. working, dyeing, dressing, machining, and selling of seal and other skins;

And whereas it is deemed for the best interests of the Tnited States that the business of handling and selling the seal and fox skins taken and to be taken as aforesaid. in accordance with the act of A pril 21,1910 (36 stat. 32(i). and the act of 1 ugu: 24,1912 (37 Stat 499 ), be conducted by the secretary of commerce through the ageney of the said Fouke Fur Co.:

Now, therefore, this agreement made and entered into this 15th day of February, 1921. by and between the United States of America (hereinafter designated as the Government), by joshua II. Alexander, iecretary of commerce, party of the first part, and the Fouke Fur Co. (hereinater designated as the company), party of the second part.

Witnesseth, that for and in consideration of the covenants and acreenents hereinafter stated, the Government will turn over to or ship to the company at st. Louis, Mo. such sealskins and fox skins as are under its control or are taken annually by it at the Pribilof Islands and are arailal, le for sale hy the foremment, and the company will receive and accept the same at said city of St. Louis. Mo... as a consignment from the Government, to be sold by the company at public auction in the city of st. Louis; or to have dressed and dyed and then sold by the company at pullic auction in the city of St. Youis, on hehali of the (iovermment. upon the terms and conditions following, that is to say:

First. All freight charges and other expenses involved in the transportation of said sealskins and said fox skins from san Francisen, ('alif.. Seattle. Wash., or other port to which they may be brought, to the warehouse of the rompany at sit. Louis, including advance charges at San Francisco, (alif., seattle. Wash.. or other port to which they may be brought, and carriage at st. Louis, shall he paid hy the company and deducted from the proceeds of sales as hereinafter provided.

Second. Aiter the receipt of said sealskins and said fox skins at the warhonse of the company in St. Louis, and while in the possession of the comprany thereater and until they shall have been dyed, dressed, and sold, as prosided for herein, they shall he kept insured by the said company against any lns by tire and all due and customary precautions shall be taken hy the rompany for the safe-keeping of said skins and to prevent deterioration, the expenses thereof being borne by the company. 
Third. Said sealskins and said fox skins shall be sold by the company at public auction in the city of St. Louis, Mo., on such terms as will require payment therefor in not to exceed 90 days, and at such time or times as shall be deemed most advantageous to the Government. The company shall promptly advise the Secertary of ('ommerce of the time when it proposes to make such sale, and the Secretary of Commerce shall have the right, not less than 15 days before the time at which said sale is appointed to be held, to change the time and determine when such sale shall occur. At such sale or sales, the Secretary of ('ommerce or his duly authorized representative shall have the right to withdraw from sale said sealskins and fox skins or any lot or lots thereof, at any time before the same shall be actually sold.

Fourth. The company shall render to the Secretary of Commerce just and proper accounts of the gross proceeds of any and all sales of said sealskins and said fox skins offered by it, and of all sums received and paid by it, and will keep such accounts in proper books which shall be deemed the property of the Government. And the Secretary of Commerce shall at all times be at liberty, either personally or by agent duly authorized by him, to inspect and carry away such books, or demand and carry away copies of, or extracts from, such books.

Fifth. The company shall remit and pay to the Secretary of Commerce the net proceeds of any and all sales of said seal and said fox skins effected by it, such net proceeds to be determined as hereinafter provided, within 100 days after such sales shall have occurred.

Sixth. If the Government shall deem it advisable or necessary, the company will, during the term of this contract, during the fur-seal killing season each year, send to the Pribilof Islands not to exceed five experts experienced in the killing and skinning of seals and the salting of sealskins, as the Government may direct, for the purpose of cooperating with the department's representatives and of instructing and assisting the natives and others engaged in such work, in order that skins taken by the Government may be secured and handled in the best possible manner.

Seventh. That the salaries and compensation of such experts, as well as all other expenses incidental to their employment, shall be paid by the company, and all traveling and subsistence expenses from St. Louis to the Pribilof Islands and return shall be borne hy the Government, and the Government shall furnish said experts with board and lodging while on the said Pribilof Islands for the purposes aforesaid.

Eighth. That the company will, during the term of this contract, on or before the 1st day of May in each year, advance to a representative of the Government, to be designated by the Secretary of Commerce, such sum as shall be determined by the Government to be necessary to enable its representatives to pay such compensation for services as shall be fixed by the said Secretary of Commerce to persons engaged in killing and skimning foxes and seals and salting and handling said skins, or otherwise employed in connection therewith, preparatory to the sale of said skins, it being understood and agreed that the company will deduct from any payment of net proceeds to be made as provided for in paragraph fifth the advance made by it as aforesaid.

Ninth. In making up the account of the sales of said sealskins and said fox skins, and in order to ascertain the net proceeds thereof, the company shall be entitled to receive and retain out of the gross proceeds of said sales the commission hereinafter specified as and for full compensation for all services it may render and all expenses it may incur in connection with the storage, sorting, protecting, insurance as aforesaid, and proper care of all of said skins, and advertising and sale thereof, and shall also deduct all amounts advanced to pay costs of and expenses incidental to the transportation as provided in paragraph first hereof, and for any time in excess of six months during which skins are held by the company by reason of the postponement of any sale as hereinbefore provided for, the company shall be allowed to charge for storage at regular market rates, and the amount remaining, subject to the provisions hereinafter stated, shall be the net proceeds of said sales and payalle to the Secretary of Commerce.

Tenth. All casks in which said sealskins and said fox skins are packed, and all surplus salt which may come from said skins, shall, if the same has a salable value, be sold by the company and full amount of the proceeds shall be accounted for and paid over by the company to the Secretary of ('ommerce when settlement for the proceeds of the sale of said skins shall be made.

Eleventh. All contracts made by the company relating to said sealskins and said fox skins for their transportation or their care and protection pending sale, and for the sale and delivery thereof, and all contracts or policies of insurance against risks of transportation, fire, and otherwise, may be made by the company in its own name, but the same shall inure to the benefit of the Government; and all claims for 
loss of said sealskins and said fox skins, or any or either of them, or for damare thereto, which the company may be entitled to make or shall collect against common carriers; or loss or dimage by reason of failure to sately and duly transport and deliver sur.h skins, shall likewise inure to the benefit of and be paid to the (rovernment, less the legal charge for collection.

Twelfth. When directed by the Secretary of Commerce, the company shall secure marine and transportation insurance, the necessary cost thereof to be paid by the company and deductesl irom any payment of net proceeds to be mate as provided for in the fifth paragraph hereof.

Thirteenth. During the term of the existence of this contract, the following commissions shall be paid to the company as and for full compensation for all services it may render and all expenses it may incur in connection with the storage (except skins ortered held lonerer than six months as provided in pararaph ninth), sortiner, protection, insurance as provided in paragraph second, and the proper care of all said skins, and the advertising and sale thereof:

1. If the total number of sealskins and fox skins combined to be sold, consigned in anv one year to the company, shall not exceed 20.000 , the rate of commission ficured on the basis aforesaid shall be 4 ner cent.

2. If such total number shall exceed 20,000 , but not exceed 30,000 , the rate of such commission shall be $33_{4}^{3}$ per cent.

3. If such total number shall exceed 30.000 , but not exceed 40,000 , the rate of such commission shall be $3 !$ per cent.

4. If such total number shall exceed 40,000 , but not exceed 50,000 , the rate of such commission shall be $3 \frac{1}{\text { i per cent. }}$

5 . If such total number shall exceed 50,000 , the rate of such commission shall be 3 per cent.

Fourteenth. In consideration of the premises, and especially of the compensation provided for in this agreement, the company hereby agrees, subject to the provisions of paragraph 15 hereof, to to or have done the dressing, dyeing, and machining of all sealskins turned over to it for this purpose, in accordance with the process heretofore used in the dyeing, dressing, and machining of skins by the Gibbins \& L.ohn Dressing \& Dyeing Co., or to have the said dyeing, dressing and machining done in such other manner as shall be satisfactory to the Secretary of Commerce.

Fifteenth. For the dyeing, dressing, and machining of sealskins, as provided for in paragraph 14, the company shall be entitled to make charges in accordance with the following graduated scale of prices, which are based on the total number of sealskins taken by the Government each calendal year, the amounts of which charges the company is authorized and directed to retain from the proceeds of the sale of any and all such skins sold by the company for account of the Government. Such skins as are credited to or belong to the Governments of Great Britain and Japan, under the convention of July 7,1911 , which are turned over to the company for dyeing, dressing, and machining, shall be included in the number on which the compensation is based.

The graduated scale of prices herein referred to, arranged in the form of a table, shows the price per skin. 'The figures in the first column indicate the minimum and maximum number of sealskins taken by the Government, on which the unit price is hased. The figures at the top of the second, third, fourth, fifth, and sicth columns indicate the years in which the skins may be taken.

\begin{tabular}{|c|c|c|c|c|c|}
\hline & 1921 & 1922 & 1923 & 1924 & 1925 \\
\hline 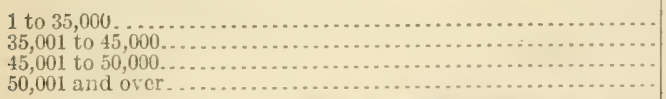 & $\begin{array}{r}\$ 16.50 \\
16.50 \\
16.50 \\
16.50\end{array}$ & $\begin{array}{r}\$ 15.50 \\
15.50 \\
15.50 \\
15.50\end{array}$ & $\begin{array}{r}\$ 14.50 \\
14.00 \\
13.50 \\
13.00\end{array}$ & $\begin{array}{l}\$ 13.50 \\
13.00 \\
12.50 \\
12.00\end{array}$ & $\begin{array}{l}\$ 12.50 \\
12.00 \\
11.50 \\
11.00\end{array}$ \\
\hline
\end{tabular}

Upon the execution of this contract the company shall ardvance and fay to the Fumsten Bros. \& Co. $\$ 200,0(0)$, which is to be in full paiment of any and all churges which the said Funsten Bros. \& Co. may have made or may make to cover advances made by the said Funsten Bros. \& (C). in connection with the transportation insurance. storace. dyeing, dressing, and machining of all skins now belonging to the finvernment. and shall obtain and deliver to the secretary of Commerce a receipt from the said Funsten Bros. \& Co. showing that the said 200, (b) has heren receired hy it and that it releases and discharges the Government from any other or further fayment in conmection with the execution of any and all of the contracts and agreements herefolore existing by and between the United States and the said Funsten Bros. \& Co. 
The company further specially agrees that no charge shall be made for the transportation, insurance, storage, dyeing, dressing, and machining of the first 16,000 skins which may be sold by the company for and on account of the Government, the company being entitled to charge for the dyeing, dressing, and machining of all skins, exclusive of the first 16,000, sold by it for and on account of the Government, the prices as fixed in the table of prices above in this paragraph set forth, in consideration of which special agreement, the Government specially agrees that, from the first sale of skins made by the company for and on account of the Government, the company shall deduct and retain, in addition to the commissions provided for in paragraph thirteenth hereof, $\$ 125,000$, and from the proceeds of the second sale of skins made by the company for and on account of the Government, the company shall deduct and retain, in addition to the commissions provided for in paragraph thirteenth hereof. $\$ 75,000$.

The prices fixed in this paragraph for dyeing, dressing, and machining of sealskins for the year 1925 shall continue in force after the calendar year 1925 until the termination of this contract unless by mutual consent expressed in writing the said prices are raised or lowered to meet changed conditions.

Sixteenth. That the company will, during the term of this agreement, at its own expense and without any cost or charge whatsoever to the Government, by inserting advertisements in the proper technical press and by circulating, distributing, and mailing circulars, bulletins, letters, etc., and by other proper methods, advertise the sale of and the quality and advantages of sealskins dressed and dyed by the said process, with the view of informing the public as to the merits of such process and of inducing a more general use of sealskins than prevails at the present time

Seventeenth. If required by the Secretary of Commerce, the company agrees to furnish a bond in such sum and with such surety or sureties as shall be satisfactory to the Secretary of Commerce, conditioned upon the faithful performance of each and every covenant and agreement to be performed by the company under the provisions hereof.

Eighteenth. Unless sooner terminated by agreement under seal of the parties hereto or otherwise terminated by any legal process, this contract shall continue in force and effect until the 15th day of February, 1931.

Nineteenth. It is understood and agreed hy the parties hereto that this contract and all the provisions thereof shall be subject to any legislation that may hereafter be enacted by Congress relative to the subject matter hereof. and also to such rules and regulations as may be adopted, from time to time. by the Secretary of Commerce.

Twentieth. Pursuant to section 3741 of the Revised Statutes of the United States, it is further agreed that no Member of or Delegate to Congress, or Resident Commissioner, or any officer or employee of the Government or other person whose name is not at this time disclosed, shall be admitted to any share or interest in this contract. or to any benefit to arise therefrom; and that this contract shall be subject in all respects to the provisions of sections 114, 115, and 116 of the Criminal Code of the United States, so far as the same may be applicable.

Signed in duplicate by the parties hereto the day and year first above written.

[SEAL].

United States of America,

By Joshua W. Alexanner. Secretary of Commerce.

The Fouke Fur Co. (Inc.)

By P. B. Fouke, President.

Signed, sealed, and delivered in the presence of:

E. R. MAgIF.

H. M. SMITH.

E. F. SWEET.

MEMORANDUM AGREEMENT.

Entered into by the Secretary of Commerce on behalf of the United States, and P. B. Fouke, president of the Fouke Fur Co., relative to contract entered into on the 15 th day of February, 1921, concerning the sale of fox skins and the dyeing, dressing, machining, and sale of sealskins from the Pribilof Islands, Alaska.

Referring to contract dated February 15,1921 , by and between the United States of America, by Joshua W. Alexander, Secretary of Commerce, and the Fouke Fur Co., by P. B. Fouke, president, for the sale of fox skins, and the dyeing, dressing, machining, and sale of sealskins, the undersigned mutually agree that said contract, referred to above, be modified in the following particulars: 
1. The first paragraph of the terms and conditions of the contract referred to above is modified to read as follows: "The freight charges and other expenses involved in the transportation of said seal and fox skins from San Francisco, Calif., Seattle, Wash., or other port to which they may be brought, to the warehouse of the company at St. Louis, Mo., including any advance charges at San Francisco, Calif., Seattle, Wash., or other port to which they may be brought, shall be paid by the company when so requested by the Secretary of Commerce and such payments shall be deducted from the proceeds of sales as provided for in the contract of February 15, 1921. The Government, however, reserves the right to transport said seal and fox skins on Government bill of lading and to pay for such shipments from the proceeds of sales."

2. The following paragraph is hereby incorporated and made a part of the terms and conditions of the contract above referred to:

"Eight $(a)$. It is understood and agreed by the parties hereto that the company will, on request of the Secretary of Commerce, assume and pay all necessary expenses for supplies and materials, including salt, casks, twine, and other articles required in the taking, curing, handling, and shipment of seal and fox skins and will deduct such payments from any proceeds of sales of said seal and fox skins."

The parties hereto further covenant and agree that nothing contained herein shall be construed as altering, affecting, or modifying any of the provisions, terms, or conditions of original contract above referred to not herein expressly altered, affected, or modified.

Signed in duplicate by the parties hereto this 25th day of February, 1921.

United States Department of Commerce, By Joshua W. Alexander,

Fouke Fur Company.

Secretary of Commerce.

By P. B. Fouke,

Witnesses:

President

William B. Yancey.

A. Murphy. 

LIBRARY OF CONGRESS 